\title{
Energy Balance and Prostate Cancer
}

\section{Citation}

Dickerman, Barbra. 2018. Energy Balance and Prostate Cancer. Doctoral dissertation, Harvard University, Graduate School of Arts \& Sciences.

\section{Permanent link}

http://nrs.harvard.edu/urn-3:HUL.InstRepos:41121220

\section{Terms of Use}

This article was downloaded from Harvard University's DASH repository, and is made available under the terms and conditions applicable to Other Posted Material, as set forth at http:// nrs.harvard.edu/urn-3:HUL.InstRepos:dash.current.terms-of-use\#LAA

\section{Share Your Story}

The Harvard community has made this article openly available.

Please share how this access benefits you. Submit a story.

Accessibility 


\section{Energy Balance and Prostate Cancer}

A dissertation presented by

\section{Barbra Dickerman}

to

The Department of Epidemiology

in partial fulfillment of the requirements

for the degree of

Doctor of Philosophy

in the subject of

Population Health Sciences (Concentration: Epidemiology)

Harvard University

Cambridge, Massachusetts

August 2018 
(C) 2018 Barbra Dickerman

All rights reserved. 


\title{
Energy Balance and Prostate Cancer
}

\begin{abstract}
Obesity is associated with a higher risk of advanced prostate cancer and poorer prognosis after diagnosis. Those with the same body mass index (BMI) may differ in their underlying fat distribution and metabolic health, but the impact of these subphenotypes on subsequent prostate cancer risk remains largely unknown. Further, physical activity after prostate cancer diagnosis may improve clinical outcomes, but no randomized trial to date has reported on physical activity and survival among prostate cancer survivors. In the absence of this data, cutting-edge methods for causal inference combined with high-quality observational data are needed to evaluate the survival impact of adhering to current guidelines for physical activity.

In Chapter 1, we examined the association between body fat distribution and prostate cancer risk among Icelandic men in the AGES-Reykjavik Study. Our findings suggest that visceral and thigh subcutaneous adiposity are the most important fat depots for risk of advanced and fatal prostate cancer. Among leaner men based on BMI, visceral adiposity was the fat depot most strongly associated with both advanced and fatal prostate cancer. These findings may help elucidate underlying mechanisms and target intervention strategies.
\end{abstract}

In Chapter 2, we emulated a target trial of guideline-based physical activity interventions and 10-year survival among US men with nonmetastatic prostate cancer in the Health Professionals Follow-up Study. We estimated that these men would have 
experienced clinically meaningful reductions in mortality had they all followed current physical activity recommendations until the development of conditions limiting physical ability. These findings may help guide clinical recommendations for prostate cancer patients and future trial design.

In Chapter 3, we applied metabolomics to identify plasma metabolites associated with various adiposity measures and evaluate the association between metabolically-defined obesity and advanced prostate cancer risk. We found that BMI, waist circumference, and derived fat mass were associated with a broad range of metabolic alterations. While neither adiposity nor metabolic scores were associated with risk of advanced prostate cancer, the identified metabolites may inform future research on the mechanisms linking obesity with various disease outcomes. 


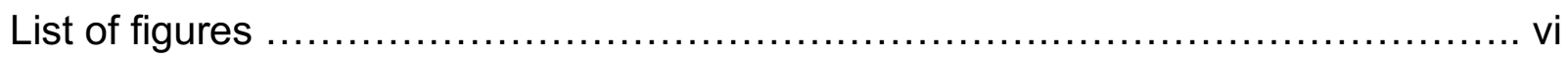

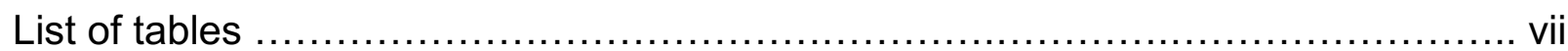

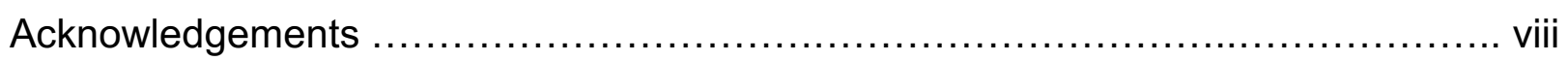

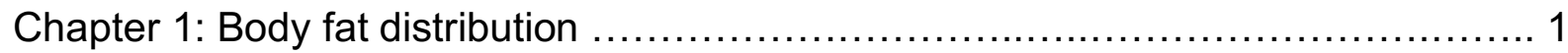

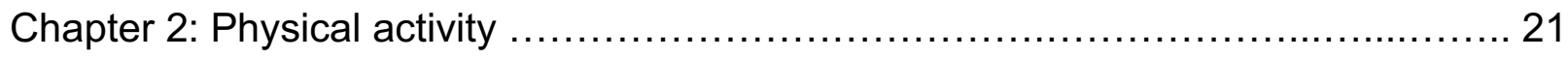

Chapter 3: Metabolomics ........................................................... 41

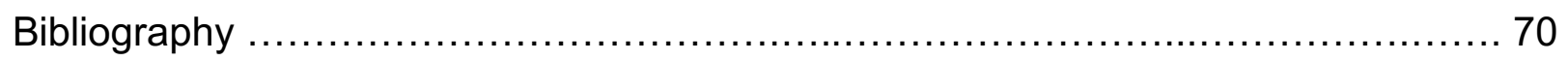

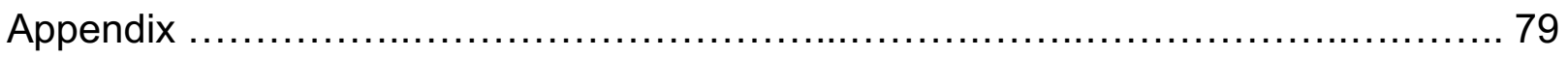




\section{LIST OF FIGURES}

Figure 1.1 Flowchart for selection of study participants from the Health

Professionals Follow-up Study

Figure 1.2 Estimated survival curves under physical activity strategies among

men with nonmetastatic prostate cancer, Health Professionals Follow-up Study ...... 35

Figure 2.1 BMI-metabolic groups and advanced prostate cancer ....................6. 62

Figure 2.2 Waist circumference-metabolic groups and advanced prostate cancer ... 63

Figure 2.3 Fat mass-metabolic groups and advanced prostate cancer ................ 64 
Table 1.1 Age-standardized characteristics of 1,832 men at entry into the AGES-

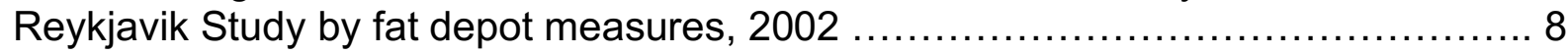

Table 1.2 Spearman correlation between adiposity measures at baseline for men

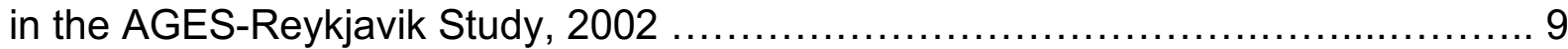

Table 1.3 Association between adiposity measures and risk of prostate cancer among men in the AGES-Reykjavik Study, 2002-2015

Table 1.4 Association between abdominal visceral fat and risk of prostate cancer among 1832 men in the AGES-Reykjavik Study, 2002-2015: By BMI

Table 2.1 Baseline characteristics of 2299 eligible participants after diagnosis with nonmetastatic prostate cancer, Health Professionals Follow-up Study, 1998-2010 .. 33 Table 2.2 Estimated risk of all-cause mortality under several physical activity strategies among men with nonmetastatic prostate cancer, Health Professionals Follow-up Study. All strategies excuse men from following the recommended physical activity levels after development of functional impairment, metastasis, myocardial infarction, stroke, congestive heart failure, or amyotrophic lateral sclerosis........... 34

Table 2.3 Estimated risk of all-cause mortality under several physical activity strategies among men with nonmetastatic prostate cancer, Health Professionals Follow-up Study. All strategies excuse men from following the recommended physical activity levels after development of functional impairment, metastasis, or a serious chronic condition

Table 3.1 Baseline characteristics of participants from nested case-control studies of various outcomes, Health Professionals Follow-up Study

Table 3.2 Metabolites associated with adiposity measures (FDR p-value $<0.05$ and $|r| \geq 0.15$ ) among controls from nested case-control studies, Health Professionals Follow-up Study

Table 3.3 Estimated odds ratios (OR) and 95\% confidence intervals (Cls) of advanced prostate cancer by adiposity measures and metabolic scores (predicted adiposity using metabolites), Health Professionals Follow-up Study ....... 58 Table 3.4 Stratified analysis by time to diagnosis: Estimated odds ratios (OR) and $95 \%$ confidence intervals (Cls) of advanced prostate cancer by self-reported adiposity and metabolic scores (predicted adiposity using metabolites), Health Professionals Follow-up Study 
I am deeply grateful for Dr. Lorelei Mucci, my advisor, mentor, and advocate, for her tremendous support and guidance throughout my doctoral training; Dr. Miguel Hernán for introducing me to a framework for causal inference that fundamentally changed the way I think about data and for providing me with many teaching opportunities, which have been a highlight of my doctoral experience; Dr. Edward Giovannucci, Dr. Brian Healy, and Dr. Unnur Valdimarsdottir, for their generous investment of time and brilliant insights that strengthened my thesis work; my coauthors and the prostate cancer epidemiology group for shaping my development as an epidemiologist; Dr. Meir Stampfer for his invaluable insights and incredible generosity; the National Institute of Health Training Grant in Cancer Epidemiology for supporting my work; the participants and staff of the AGES-Reykjavik Study and Health Professionals Follow-up Study, without whom these investigations would not be possible; Dr. Jianghong Liu, my first research mentor who encouraged me to pursue an academic career; MK Downer, Krystal Cantos, and Samantha Molsberry, for making everything more fun (even studying for the qualifying exam); and Aaron for being my biggest supporter. 


\section{Body fat distribution and prostate cancer risk and mortality in the AGES-Reykjavik Study}

Barbra A. Dickerman, Johanna E. Torfadottir, Unnur A. Valdimarsdottir, Edward Giovannucci, Kathryn M. Wilson, Thor Aspelund, Laufey Tryggvadottir, Lara G.

Sigurdardottir, Tamara B. Harris, Lenore J. Launer, Vilmundur Gudnason, Sarah C. Markt and Lorelei A. Mucci 


\section{ABSTRACT}

Background: Obesity is associated with a higher risk of advanced prostate cancer prostate cancer, but the role of body fat distribution remains unclear.

Objective: To prospectively investigate objectively measured adiposity and risk of prostate cancer.

Design, setting, and participants: From 2002-2006, a population-based sample of men in the AGES-Reykjavik Study underwent baseline computed tomography (CT) imaging of fat deposition, bioelectric impedance analysis, and measurement of body mass index (BMI) and waist circumference. We followed 1,832 men through linkage with nationwide cancer registries for incidence of total $(n=172)$, high-grade (Gleason grade $\geq 8)(n=43)$, advanced $(\geq \mathrm{cT3}$ /N1/M1 at diagnosis or fatal prostate cancer over follow-up) $(n=41)$, and fatal $(n=31)$ prostate cancer through 2015.

Outcome measurements and statistical analysis: Cox regression was used to evaluate the association between adiposity measures and prostate cancer outcomes.

Results: Among all men, the fat depots most strongly associated with risk of advanced and fatal prostate cancer were visceral (hazard ratio [HR] 1.31 per 1 SD increase, 95\% confidence interval [CI]: 1.00-1.72) and thigh subcutaneous adiposity (HR 1.37 per 1 SD increase, $95 \% \mathrm{Cl}: 1.00-1.88)$, respectively. Among leaner men based on BMI, visceral adiposity was the fat depot most strongly associated with both advanced and fatal prostate cancer. BMI and waist circumference were associated with a higher risk of advanced and fatal prostate cancer. No adiposity measures were associated with total or high-grade prostate cancer. 
Conclusions: These findings suggest that visceral and thigh subcutaneous adiposity are the most important fat depots for risk of advanced and fatal prostate cancer, and, further, that visceral adiposity is the most important fat depot for these outcomes among leaner men based on BMI. This may help elucidate underlying mechanisms and target intervention strategies.

\section{INTRODUCTION}

Obesity is associated with a higher risk of advanced prostate cancer and poorer prognosis after diagnosis. ${ }^{1}$ Emerging evidence suggests that the specific distribution of body fat may be an important prognostic factor for prostate cancer outcomes. ${ }^{2-5}$ Body fat distribution is of interest because it may be a marker for different metabolic, hormonal, and inflammatory milieus that play a role in prostate carcinogenesis. ${ }^{3,6-13}$ The identification of adiposity phenotypes at highest risk of aggressive prostate cancer may therefore help elucidate the mechanisms linking obesity with aggressive disease and target corresponding intervention strategies.

To date, few studies have investigated directly measured body fat distribution and prostate cancer risk. Cross-sectional and retrospective studies have reported associations between computed tomography (CT) measures of visceral fat and total ${ }^{4}$ and high-grade ${ }^{5}$ prostate cancer. However, these studies were limited by small samples and the potential that the disease or its treatment may have influenced adiposity. ${ }^{4,5}$ Further, the association between other fat depots and prostate cancer outcomes remains unclear.

Here we undertook the first prospective study of CT-measured fat distribution and risk of prostate cancer and measures of aggressive disease. 


\section{MATERIALS AND METHODS}

\section{Study population}

We leveraged data from the AGES-Reykjavik study, a longitudinal populationbased study in Iceland described in detail elsewhere. ${ }^{14}$ From 2002-2006, participants underwent a comprehensive baseline examination involving a medical history, physical examination, imaging studies, and questionnaires on health-related behaviors. At baseline, we excluded those with a history of cancer $(n=453)$, missing CT data $(n=136)$, or a $\mathrm{BMI}<18.5 \mathrm{~kg} / \mathrm{m}^{2}(\mathrm{~N}=17)$, leaving 1,832 men in our analysis. The study was approved by the Icelandic Ethical Review Board and the Icelandic Data Protection Authority.

\section{Adiposity measures and covariates}

Adiposity was assessed at baseline. Participants underwent CT imaging for assessment of fat area in the abdomen (visceral and subcutaneous) and thigh (intermuscular and subcutaneous). CT imaging is the gold standard for measuring fat distribution, ${ }^{15}$ and the internal reliability of this measure was excellent ( $C V<5 \%$ for all fat depot measures). Imaging was performed with a 4-row detector system (Sensation; Siemens Medical Systems, Erlangen, Germany). Abdominal visceral and subcutaneous fat areas $\left(\mathrm{cm}^{2}\right)$ were measured from a single 10-mm trans-axial section at the L4/L5 vertebrae. Visceral fat was distinguished from subcutaneous fat by tracing along the fascial plane defining the internal abdominal wall. Thigh intermuscular and subcutaneous fat areas were measured from a single 10-mm trans-axial section using a $120-k V$ peak at the femoral midpoint by manually drawing a line along the deep fascial 
plane surrounding the thigh muscles. ${ }^{16}$ Analysis of the CT images was performed using specialized software developed at the University of California, San Francisco. Total body fat was measured by bioelectrical impedance. Height, weight, and waist circumference were measured by trained technicians. BMI was calculated as weight $(\mathrm{kg}) /$ height $(\mathrm{m})^{2}$. We obtained information on lifestyle and clinical covariates from the baseline questionnaire.

\section{Outcome ascertainment}

Record linkage to the nationwide Icelandic Cancer Registry through unique identification numbers was used to identify prostate cancer diagnosed from study entry through December 31, 2015. We also obtained information on all-cause and prostate cancer -specific deaths over the study period from the Directorate of Health. Cancer registration is mandatory and estimated completeness is very high (99.2\%). ${ }^{17}$ Over $98 \%$ of prostate cancer diagnoses were morphologically verified. ${ }^{17}$ Incident prostate cancer was categorized as total, high-grade (Gleason grade $\geq 8$ ), advanced ( $\geq c T 3 b$ or N1 or M1 at diagnosis, or fatal prostate cancer over follow-up), and fatal (which was also included in the advanced category). We were missing data on stage and grade for 13 (7.6\%) cases.

\section{Statistical analysis}

We estimated the correlation between adiposity measures by calculating Spearman correlation coefficients. We also conducted partial correlation analysis adjusting for age. 
We used Cox regression to estimate hazard ratios (HRs) and 95\% confidence intervals (Cls) for total, high-grade, advanced, and fatal prostate cancer. We followed men from the date of the baseline examination until the incident prostate cancer outcome of interest, death, or administrative end of follow-up (December 31, 2015), whichever happened first. We adjusted for the baseline covariates: age, family history of prostate cancer, smoking status, education, frequency of moderate/vigorous physical activity during youth and midlife, and presence of a physician visit over the past year. Our primary analyses did not adjust for alcohol consumption because of inconsistent findings for a link with prostate cancer; however, estimates were qualitatively similar with adjustment for alcohol (data not shown). Models for fat depots and waist circumference were additionally adjusted for height (continuous). In sensitivity analyses for the fat depot models, we additionally adjusted for BMI and mutually adjusted for all fat depots. Missing data for categorical covariates were assigned to the referent group (smoking status, $n=2$; education, $n=19$, physical activity, $n=111$; physician visit, $n=35$ ).

We further conducted pre-specified stratified analyses to evaluate whether the association between fat distribution and prostate cancer varied by BMI (dichotomized at the median; $<27 \mathrm{vs.} \geq 27 \mathrm{~kg} / \mathrm{m}^{2}$ ). This cut-off was selected to optimize case distribution and power for analyses in each stratum. Tests for heterogeneity were performed using likelihood ratio tests comparing models with and without a product term between the exposure of interest and BMI. Finally, we conducted sensitivity analyses (1) excluding men older than 80 years at study entry and (2) excluding the first five years of follow-up to address potential reverse causation.

Analyses were conducted using SAS 9.3 (SAS Institute, Inc., Cary, NC). 


\section{RESULTS}

Among 1,832 men, there were 172 prostate cancer diagnoses, including 31 prostate cancer-specific deaths, over the study period. Of the incident prostate cancer diagnoses, 41 were advanced and 43 were high-grade tumors. Median follow-up time was 10.1 years until prostate cancer diagnosis and 10.4 years until prostate cancer death.

Table 1.1 shows baseline characteristics of the men by fat depot measures dichotomized at the median. Those with higher visceral fat had a higher BMI and waist circumference, lower physical activity during youth and midlife, and were less likely to be current smokers. Similar associations were seen for higher levels of other fat depots.

Appendix Table 1.1 shows the distribution of adiposity measures. At baseline, the median BMI was $27 \mathrm{~kg} / \mathrm{m}^{2}$ and median waist circumference was $102 \mathrm{~cm}$. Table 1.2 shows the Spearman correlation coefficients between adiposity measures. BMI was highly correlated with waist circumference $\left(r_{s}=0.87\right)$ and total body fat $\left(r_{s}=0.84\right)$. Of the fat depots, abdominal subcutaneous fat was highly correlated with BMI and waist circumference $\left(r_{\mathrm{s}}=0.82\right.$ and $r_{\mathrm{s}}=0.83$, respectively); visceral fat was correlated with BMI and waist circumference to a lesser extent $\left(r_{s}=0.69\right.$ and $r_{s}=0.73$, respectively). Estimates were similar after adjusting for age (data not shown). 


\begin{tabular}{|c|c|c|c|c|c|c|c|c|}
\hline & \multicolumn{2}{|c|}{ Abdominal visceral } & \multicolumn{2}{|c|}{ Abdominal subcutaneous } & \multicolumn{2}{|c|}{ Thigh intermuscular } & \multicolumn{2}{|c|}{ Thigh subcutaneous } \\
\hline & $\begin{array}{r}<\mathrm{M} \\
\mathrm{N}=908\end{array}$ & $\begin{array}{r}\geq \mathrm{M} \\
\mathrm{N}=924\end{array}$ & $\begin{array}{r}<M \\
N=907\end{array}$ & $\begin{array}{r}\geq M \\
N=925\end{array}$ & $\begin{array}{r}<M \\
N=887\end{array}$ & $\begin{array}{r}\geq \mathrm{M} \\
\mathrm{N}=945\end{array}$ & $\begin{array}{r}<\mathrm{M} \\
\mathrm{N}=901\end{array}$ & $\begin{array}{r}\geq M \\
N=931\end{array}$ \\
\hline Follow-up time ${ }^{2}$ (years) & $8.6(3.8)$ & $8.8(3.5)$ & $8.6(3.8)$ & $8.8(3.5)$ & $8.9(3.7)$ & $8.5(3.6)$ & $8.7(3.7)$ & $8.6(3.6)$ \\
\hline Age at entry (years) & $76.7(5.4)$ & $75.9(5.2)$ & $76.9(5.5)$ & $75.6(5.1)$ & $76.0(5.4)$ & $76.5(5.3)$ & $76.6(5.4)$ & $76.0(5.3)$ \\
\hline Height $(\mathrm{cm})$ & $175.0(6.0)$ & $176.0(6.2)$ & $175.0(6.0)$ & $176.0(6.2)$ & $175.1(6.0)$ & $175.9(6.3)$ & $175.2(6.0)$ & $175.8(6.3)$ \\
\hline Body mass index $\left(\mathrm{kg} / \mathrm{m}^{2}\right)$ & $24.9(2.8)$ & $28.9(3.4)$ & $24.5(2.4)$ & $29.3(3.1)$ & $25.2(3.1)$ & 28.6(3.6) & $25.2(2.9)$ & 28.7(3.6) \\
\hline Waist circumference $(\mathrm{cm})$ & $96.6(7.7)$ & $108.3(9.2)$ & $95.6(6.8)$ & $109.2(8.5)$ & $98.0(8.9)$ & $106.7(9.9)$ & $97.5(8.0)$ & $107.3(9.9)$ \\
\hline Total fat mass $(\mathrm{kg})$ & $14.8(4.9)$ & $22.3(6.2)$ & $14.3(4.2)$ & $22.9(5.9)$ & $15.6(5.6)$ & 21.7(6.7) & $15.4(5.2)$ & $21.8(6.6)$ \\
\hline Percent body fat & $19.1(4.7)$ & $24.7(4.2)$ & $18.8(4.3)$ & $25.1(4.1)$ & $19.7(4.9)$ & $24.2(4.8)$ & $19.5(4.7)$ & $24.3(4.7)$ \\
\hline \multicolumn{9}{|l|}{ Education } \\
\hline - Primary, \% & 16.9 & 14.9 & 16.2 & 15.6 & 16.3 & 15.7 & 15.4 & 16.6 \\
\hline - Secondary, \% & 53.4 & 53.3 & 54.0 & 52.7 & 53.0 & 53.2 & 53.5 & 52.7 \\
\hline - College, \% & 12.1 & 12.1 & 12.5 & 11.6 & 12.0 & 12.2 & 12.7 & 11.5 \\
\hline - University, \% & 16.8 & 18.4 & 16.6 & 18.6 & 17.7 & 17.8 & 17.6 & 17.9 \\
\hline \multicolumn{9}{|l|}{ Smoking status } \\
\hline - Never, \% & 32.0 & 27.0 & 29.9 & 28.9 & 30.0 & 28.3 & 31.9 & 26.9 \\
\hline - Former ${ }^{3}, \%$ & 54.0 & 64.2 & 56.5 & 61.9 & 56.2 & 62.6 & 55.5 & 63.2 \\
\hline - Current, \% & 13.9 & 8.7 & 13.5 & 9.2 & 13.7 & 9.0 & 12.6 & 9.7 \\
\hline $\begin{array}{l}\text { Frequency of moderate/vigorous } \\
\text { physical activity, } \geq 4 \text { hours/week, } \%\end{array}$ & 38.9 & 34.7 & 39.0 & 34.6 & 39.5 & 33.7 & 37.7 & 35.7 \\
\hline Family history of prostate cancer, $\%$ & 9.6 & 9.3 & 10.0 & 8.6 & 8.7 & 9.9 & 10.1 & 8.9 \\
\hline Physician visit over past 12 months, \% & 78.2 & 83.5 & 79.5 & 82.3 & 77.0 & 84.3 & 79.9 & 82.0 \\
\hline Type 2 diabetes ${ }^{4}, \%$ & 12.0 & 20.0 & 13.1 & 19.1 & 13.5 & 18.6 & 16.6 & 15.8 \\
\hline
\end{tabular}

Values are means (SD) or percentages and are standardized to the age distribution of the study population.

${ }^{1}$ Dichotomized at median: abdominal visceral $\left(195 \mathrm{~cm}^{2}\right)$ and subcutaneous $\left(193 \mathrm{~cm}^{2}\right)$; thigh intermuscular $\left(33 \mathrm{~cm}^{2}\right)$ and subcutaneous $\left(71 \mathrm{~cm}^{2}\right)$.

2 Time from enrollment to prostate cancer diagnosis, death, or end of follow-up.

${ }^{3}$ Regularly smoked at least 100 cigarettes or 20 cigars in lifetime.

${ }^{4}$ Type 2 diabetes by self-report or fasting glucose $\geq 7 \mathrm{mmol} / \mathrm{L}$. 
Table 1.2 Spearman correlation between adiposity measures at baseline for men in the AGES-Reykjavik Study, 2002

\begin{tabular}{|c|c|c|c|c|c|c|c|c|}
\hline & $\mathrm{BMI}$ & $\begin{array}{r}\text { Waist } \\
\text { circumference }\end{array}$ & $\begin{array}{r}\text { Total } \\
\text { body fat }\end{array}$ & $\begin{array}{l}\text { Percent } \\
\text { body fat }\end{array}$ & $\begin{array}{l}\text { Abdominal } \\
\text { visceral }\end{array}$ & $\begin{array}{r}\text { Abdominal } \\
\text { subcutaneous }\end{array}$ & $\begin{array}{r}\text { Thigh } \\
\text { intermuscular }\end{array}$ & $\begin{array}{r}\text { Thigh } \\
\text { subcutaneous }\end{array}$ \\
\hline BMI & 1.00 & & & & & & & \\
\hline Waist circumference & 0.87 & 1.00 & & & & & & \\
\hline Total body fat & 0.84 & 0.87 & 1.00 & & & & & \\
\hline Percent body fat & 0.71 & 0.77 & 0.95 & 1.00 & & & & \\
\hline Abdominal visceral & 0.69 & 0.73 & 0.70 & 0.65 & 1.00 & & & \\
\hline Abdominal subcutaneous & 0.82 & 0.83 & 0.81 & 0.75 & 0.55 & 1.00 & & \\
\hline Thigh intermuscular & 0.57 & 0.55 & 0.57 & 0.53 & 0.38 & 0.53 & 1.00 & \\
\hline Thigh subcutaneous & 0.60 & 0.58 & 0.59 & 0.56 & 0.36 & 0.69 & 0.34 & 1.00 \\
\hline
\end{tabular}


Visceral adiposity was the fat depot measure most strongly associated with risk of advanced prostate cancer (HR 1.31 per 1 standard deviation [SD] increase, 95\% Cl: 1.00-1.72) (Table 1.3). Thigh subcutaneous adiposity was the fat depot most strongly associated with risk of fatal prostate cancer (HR 1.37 per 1 SD increase, 95\% Cl: $1.00-$ 1.88) (Table 1.3). Mutual adjustment for all fat depots did not qualitatively change these results (HR for advanced prostate cancer 1.31 per $1 \mathrm{SD}$ increase in visceral adiposity, 95\% Cl: 0.96-1.80; HR for fatal prostate cancer 1.42 per $1 \mathrm{SD}$ increase in thigh subcutaneous adiposity, $95 \% \mathrm{Cl}: 0.90-2.25)$. Additional adjustment for $\mathrm{BMI}$ attenuated the estimates, particularly for the other fat depots (Appendix Table 1.2). Results for total fat mass and percent fat were similar; a 1 SD increase in either was associated with a non-statistically significant higher risk of advanced and fatal prostate cancer (Table 1.3). The association between visceral adiposity and advanced and fatal prostate cancer was stronger and statistically significant among men with a BMI $<27$ $\mathrm{kg} / \mathrm{m}^{2}$ and weaker and not significant among men with BMI $\geq 27 \mathrm{~kg} / \mathrm{m}^{2}$; however, confidence intervals were wide and tests for heterogeneity by BMI were not significant (Table 1.4).

Each $5 \mathrm{~kg} / \mathrm{m}^{2}$ increase in BMI was associated with a $50 \%$ higher risk of advanced (HR 1.52, 95\% Cl: 1.02-2.27) and fatal (HR 1.56, 95\% Cl: 0.97-2.53) prostate cancer (Table 1.3). Those who were obese $\left(\mathrm{BMI} \geq 30 \mathrm{~kg} / \mathrm{m}^{2}\right)$ had a higher risk of advanced (HR 2.54, 95\% Cl: $1.08-6.00)$ and fatal (HR 2.59, 95\% Cl: 0.90-7.45) prostate cancer compared with those with a healthy BMI (Table 1.3). Each 1 SD $(10.3 \mathrm{~cm})$ increase in waist circumference was associated with a $40 \%$ higher risk of advanced (HR 1.40, 95\% Cl: 1.04-1.89) and fatal (HR 1.45, 95\% Cl: 1.01-2.07) prostate cancer (Table 1.3). 
No adiposity measures were associated with risk of total or high-grade prostate cancer (Table 1.3). Results for all adiposity measures were qualitatively similar in sensitivity analyses excluding men older than 80 years at study entry and excluding the first five years of follow-up (data not shown). 
Table 1.3 Association between adiposity measures and risk of prostate cancer among men in the AGES-Reykjavik Study, 20022015

\begin{tabular}{|c|c|c|c|c|c|c|}
\hline & \multicolumn{4}{|c|}{ Total prostate cancer } & \multicolumn{2}{|c|}{ High-grade prostate cancer } \\
\hline & $\begin{array}{r}\text { Events/ } \\
\text { Total }\end{array}$ & $\begin{array}{l}\text { Age-adjusted } \\
\text { HR }(95 \% \mathrm{Cl})^{1}\end{array}$ & $\begin{array}{l}\text { Fully-adjusted } \\
\text { HR }(95 \% \mathrm{CI})^{2}\end{array}$ & $\begin{array}{r}\text { Events/ } \\
\text { Total }\end{array}$ & $\begin{array}{l}\text { Age-adjusted } \\
\mathrm{HR}(95 \% \mathrm{Cl})^{1}\end{array}$ & $\begin{array}{l}\text { Fully-adjusted } \\
\text { HR }(95 \% \mathrm{Cl})^{2}\end{array}$ \\
\hline \multicolumn{7}{|l|}{ CT imaging of fat depots } \\
\hline Abdominal visceral & $172 / 1832$ & $1.02(0.88,1.19)$ & $1.02(0.88,1.19)$ & $43 / 1832$ & $1.01(0.75,1.37)$ & $0.98(0.72,1.33)$ \\
\hline Abdominal subcutaneous & $172 / 1832$ & $0.96(0.82,1.12)$ & $0.97(0.83,1.13)$ & $43 / 1832$ & $1.02(0.76,1.38)$ & $1.02(0.76,1.38)$ \\
\hline Thigh intermuscular & $172 / 1832$ & $0.91(0.78,1.07)$ & $0.91(0.78,1.08)$ & $43 / 1832$ & $0.92(0.67,1.27)$ & $0.92(0.66,1.27)$ \\
\hline Thigh subcutaneous & $172 / 1832$ & $1.01(0.87,1.18)$ & $1.02(0.88,1.19)$ & $43 / 1832$ & $1.14(0.87,1.50)$ & $1.14(0.86,1.50)$ \\
\hline \multicolumn{7}{|c|}{ Bioelectric impedance analysis } \\
\hline Total fat mass & $132 / 1425$ & $1.00(0.84,1.19)$ & $0.98(0.83,1.18)$ & $35 / 1425$ & $1.01(0.72,1.41)$ & $0.98(0.69,1.40)$ \\
\hline Percent fat & $132 / 1425$ & $1.00(0.84,1.19)$ & $0.99(0.83,1.18)$ & $35 / 1425$ & $1.05(0.75,1.49)$ & $1.03(0.73,1.46)$ \\
\hline \multicolumn{7}{|c|}{ Anthropometric measurements } \\
\hline $\begin{array}{l}\text { BMI, } \\
\text { per } 5 \mathrm{~kg} / \mathrm{m}^{2} \text { Increase }\end{array}$ & $172 / 1832$ & $1.01(0.82,1.24)$ & $1.01(0.82,1.24)$ & $43 / 1832$ & $1.05(0.71,1.58)$ & $1.02(0.67,1.53)$ \\
\hline $\mathrm{BMI}<25 \mathrm{~kg} / \mathrm{m}^{2}$ & $56 / 579$ & 1 & 1 & $12 / 579$ & 1 & 1 \\
\hline $25 \leq \mathrm{BMI}<30 \mathrm{~kg} / \mathrm{m}^{2}$ & $81 / 899$ & $0.86(0.61,1.21)$ & $0.84(0.59,1.19)$ & $22 / 899$ & $1.05(0.52,2.12)$ & $0.96(0.47,1.96)$ \\
\hline $\mathrm{BMI} \geq 30 \mathrm{~kg} / \mathrm{m}^{2}$ & $35 / 354$ & $0.94(0.61,1.44)$ & $0.95(0.62,1.46)$ & $9 / 354$ & $1.06(0.44,2.53)$ & $1.00(0.42,2.43)$ \\
\hline Waist circumference & $172 / 1832$ & $1.01(0.87,1.17)$ & $1.02(0.87,1.19)$ & 43/1832 & $0.97(0.72,1.32)$ & $0.95(0.69,1.31)$ \\
\hline
\end{tabular}

HR: Hazard ratio; $\mathrm{Cl}$ : Confidence interval; BMI: Body mass index.

Continuous adiposity measures were modeled per 1 standard deviation (SD) increase unless otherwise noted. The adiposity measures and corresponding 1 SD increments are: abdominal visceral fat $\left(85.7 \mathrm{~cm}^{2}\right)$, abdominal subcutaneous fat $\left(85.6 \mathrm{~cm}^{2}\right)$, thigh intermuscular $\left(16.0 \mathrm{~cm}^{2}\right)$, thigh subcutaneous $\left(39.2 \mathrm{~cm}^{2}\right)$, total fat mass $(6.8 \mathrm{~kg})$, percent fat $(5.3 \%)$, waist circumference $(10.3 \mathrm{~cm})$.

${ }^{1}$ Adjusted for age at study entry (continuous).

${ }^{2}$ Additionally adjusted for the following variables, measured at study entry: family history of prostate cancer (yes, no), smoking status (never, former, current), education (primary/secondary, college/university), physical activity ( $\leq 3$ hours/week, $\geq 4$ hours/week), and physician visit over past 12 months (yes, no). Models for fat depots and waist circumference were additionally adjusted for height (continuous). 
Table 1.3 (Continued) Association between adiposity measures and risk of prostate cancer among men in the AGES-Reykjavik Study, 2002-2015

\begin{tabular}{|c|c|c|c|c|c|c|}
\hline & \multicolumn{4}{|c|}{ Advanced prostate cancer } & \multicolumn{2}{|c|}{ Fatal prostate cancer } \\
\hline & $\begin{array}{r}\text { Events/ } \\
\text { Total }\end{array}$ & $\begin{array}{l}\text { Age-adjusted } \\
\text { HR }(95 \% \mathrm{CI})^{1}\end{array}$ & $\begin{array}{l}\text { Fully-adjusted } \\
\mathrm{HR}(95 \% \mathrm{CI})^{2}\end{array}$ & $\begin{array}{r}\text { Events/ } \\
\text { Total }\end{array}$ & $\begin{array}{l}\text { Age-adjusted } \\
\text { HR }(95 \% \mathrm{Cl})^{1}\end{array}$ & $\begin{array}{l}\text { Fully-adjusted } \\
\text { HR }(95 \% \mathrm{Cl})^{2}\end{array}$ \\
\hline \multicolumn{7}{|l|}{ CT imaging of fat depots } \\
\hline Abdominal visceral & 41/1832 & $1.31(0.99,1.74)$ & $1.31(1.00,1.72)$ & $31 / 1832$ & $1.21(0.86,1.71)$ & $1.24(0.89,1.73)$ \\
\hline Abdominal subcutaneous & $41 / 1832$ & $1.17(0.87,1.57)$ & $1.22(0.91,1.63)$ & $31 / 1832$ & $1.18(0.84,1.66)$ & $1.26(0.89,1.78)$ \\
\hline Thigh intermuscular & $41 / 1832$ & $1.00(0.73,1.36)$ & $1.02(0.75,1.40)$ & $31 / 1832$ & $1.22(0.88,1.70)$ & $1.27(0.91,1.78)$ \\
\hline Thigh subcutaneous & $41 / 1832$ & $1.21(0.92,1.59)$ & $1.25(0.95,1.64)$ & $31 / 1832$ & $1.29(0.94,1.77)$ & $1.37(1.00,1.88)$ \\
\hline \multicolumn{7}{|c|}{ Bioelectric impedance analysis } \\
\hline Total fat mass & $32 / 1425$ & $1.17(0.83,1.65)$ & $1.17(0.83,1.67)$ & $25 / 1425$ & $1.15(0.77,1.72)$ & $1.17(0.78,1.75)$ \\
\hline Percent fat & $32 / 1425$ & $1.20(0.84,1.71)$ & $1.19(0.83,1.69)$ & $25 / 1425$ & $1.20(0.79,1.81)$ & $1.20(0.80,1.81)$ \\
\hline \multicolumn{7}{|c|}{ Anthropometric measurements } \\
\hline $\begin{array}{l}\text { BMI, } \\
\text { per } 5 \mathrm{~kg} / \mathrm{m}^{2} \text { Increase }\end{array}$ & $41 / 1832$ & $1.45(0.98,2.16)$ & $1.52(1.02,2.27)$ & $31 / 1832$ & $1.46(0.91,2.34)$ & $1.56(0.97,2.53)$ \\
\hline $\mathrm{BMI}<25 \mathrm{~kg} / \mathrm{m}^{2}$ & $10 / 579$ & 1 & 1 & $7 / 579$ & 1 & 1 \\
\hline $25 \leq \mathrm{BMI}<30 \mathrm{~kg} / \mathrm{m}^{2}$ & $18 / 899$ & $1.14(0.53,2.48)$ & $1.19(0.54,2.61)$ & $16 / 899$ & $1.53(0.63,3.73)$ & $1.68(0.68,4.14)$ \\
\hline $\mathrm{BMI} \geq 30 \mathrm{~kg} / \mathrm{m}^{2}$ & $13 / 354$ & $2.18(0.95,5.03)$ & $2.54(1.08,6.00)$ & $8 / 354$ & $2.11(0.76,5.87)$ & $2.59(0.90,7.45)$ \\
\hline Waist circumference & $41 / 1832$ & $1.32(0.98,1.77)$ & $1.40(1.04,1.89)$ & $31 / 1832$ & $1.31(0.92,1.86)$ & $1.45(1.01,2.07)$ \\
\hline
\end{tabular}

HR: Hazard ratio; $\mathrm{Cl}$ : Confidence interval; BMI: Body mass index.

Unless otherwise noted, continuous adiposity measures were modeled per 1 standard deviation (SD) increase. The adiposity measures and corresponding 1 SD increments are: abdominal visceral fat $\left(85.7 \mathrm{~cm}^{2}\right)$, abdominal subcutaneous fat $\left(85.6 \mathrm{~cm}^{2}\right)$, thigh intermuscular $\left(16.0 \mathrm{~cm}^{2}\right)$, thigh subcutaneous $\left(39.2 \mathrm{~cm}^{2}\right)$, total fat mass $(6.8 \mathrm{~kg})$, percent fat $(5.3 \%)$, waist circumference $(10.3 \mathrm{~cm})$

${ }^{1}$ Adjusted for age at study entry (continuous).

${ }^{2}$ Additionally adjusted for the following variables, measured at study entry: family history of prostate cancer (yes, no), smoking status (never, former, current), education (primary/secondary, college/university), physical activity ( $\leq 3$ hours/week, $\geq 4$ hours/week), and physician visit over past 12 months (yes, no). Models for fat depots and waist circumference were additionally adjusted for height (continuous). 
Table 1.4 Association between abdominal visceral fat and risk of prostate cancer among 1832 men in the AGES-Reykjavik Study, 2002-2015: By BMI

\begin{tabular}{|c|c|c|c|c|}
\hline & \multicolumn{2}{|c|}{ Total prostate cancer } & \multicolumn{2}{|c|}{ High-grade prostate cancer } \\
\hline & $\begin{array}{r}\text { Events/ } \\
\text { Total }\end{array}$ & $\begin{array}{l}\text { Fully-adjusted } \\
\text { HR }(95 \% \mathrm{Cl})^{1}\end{array}$ & $\begin{array}{r}\text { Events/ } \\
\text { Total }\end{array}$ & $\begin{array}{l}\text { Fully-adjusted } \\
\text { HR }(95 \% \mathrm{Cl})^{1}\end{array}$ \\
\hline \multicolumn{5}{|l|}{ Abdominal visceral } \\
\hline $\mathrm{BMI}<27 \mathrm{~kg} / \mathrm{m}^{2}$ & $99 / 981$ & $1.20(0.91,1.57)$ & $26 / 981$ & $1.31(0.76,2.24)$ \\
\hline $\mathrm{BMI} \geq 27 \mathrm{~kg} / \mathrm{m}^{2}$ & $73 / 851$ & $1.05(0.83,1.34)$ & $17 / 851$ & $0.97(0.59,1.61)$ \\
\hline p-heterogeneity & & 0.82 & & 0.92 \\
\hline \multicolumn{5}{|c|}{ Abdominal subcutaneous } \\
\hline $\mathrm{BMI}<27 \mathrm{~kg} / \mathrm{m}^{2}$ & $99 / 981$ & $0.97(0.69,1.37)$ & $26 / 981$ & $1.60(0.82,3.11)$ \\
\hline $\mathrm{BMI} \geq 27 \mathrm{~kg} / \mathrm{m}^{2}$ & $73 / 851$ & $1.12(0.88,1.42)$ & $17 / 851$ & $1.14(0.70,1.85)$ \\
\hline p-heterogeneity & & 0.51 & & 0.67 \\
\hline \multicolumn{5}{|l|}{ Thigh intermuscular } \\
\hline $\mathrm{BMI}<27 \mathrm{~kg} / \mathrm{m}^{2}$ & $99 / 981$ & $0.90(0.65,1.22)$ & $26 / 981$ & $0.86(0.46,1.63)$ \\
\hline $\mathrm{BMI} \geq 27 \mathrm{~kg} / \mathrm{m}^{2}$ & $73 / 851$ & $0.99(0.79,1.24)$ & $17 / 851$ & $1.11(0.70,1.74)$ \\
\hline p-heterogeneity & & 0.78 & & 0.63 \\
\hline \multicolumn{5}{|l|}{ Thigh subcutaneous } \\
\hline $\mathrm{BMI}<27 \mathrm{~kg} / \mathrm{m}^{2}$ & $99 / 981$ & $1.12(0.84,1.51)$ & $26 / 981$ & $1.66(0.99,2.79)$ \\
\hline $\mathrm{BMI} \geq 27 \mathrm{~kg} / \mathrm{m}^{2}$ & $73 / 851$ & $1.08(0.88,1.33)$ & $17 / 851$ & $1.19(0.80,1.78)$ \\
\hline p-heterogeneity & & 0.59 & & 0.45 \\
\hline
\end{tabular}

HR: Hazard ratio; Cl: Confidence interval; BMI: Body mass index.

Continuous adiposity measures were modeled per 1 standard deviation (SD) increase: abdominal visceral fat $\left(85.7 \mathrm{~cm}^{2}\right)$, abdominal subcutaneous fat $\left(85.6 \mathrm{~cm}^{2}\right)$, thigh intermuscular $\left(16.0 \mathrm{~cm}^{2}\right)$, thigh subcutaneous $\left(39.2 \mathrm{~cm}^{2}\right)$.

${ }^{1}$ Adjusted for the following variables, measured at study entry: age (continuous), height (continuous), family history of prostate cancer (yes, no), smoking status (never, former, current), education (primary/secondary, college/university), physical activity ( $\leq 3$ hours/week, $\geq 4$ hours/week), and physician visit over past 12 months (yes, no). 
Table 1.4 (Continued) Association between abdominal visceral fat and risk of prostate cancer among 1832 men in the AGES-Reykjavik Study, 2002-2015: By BMI

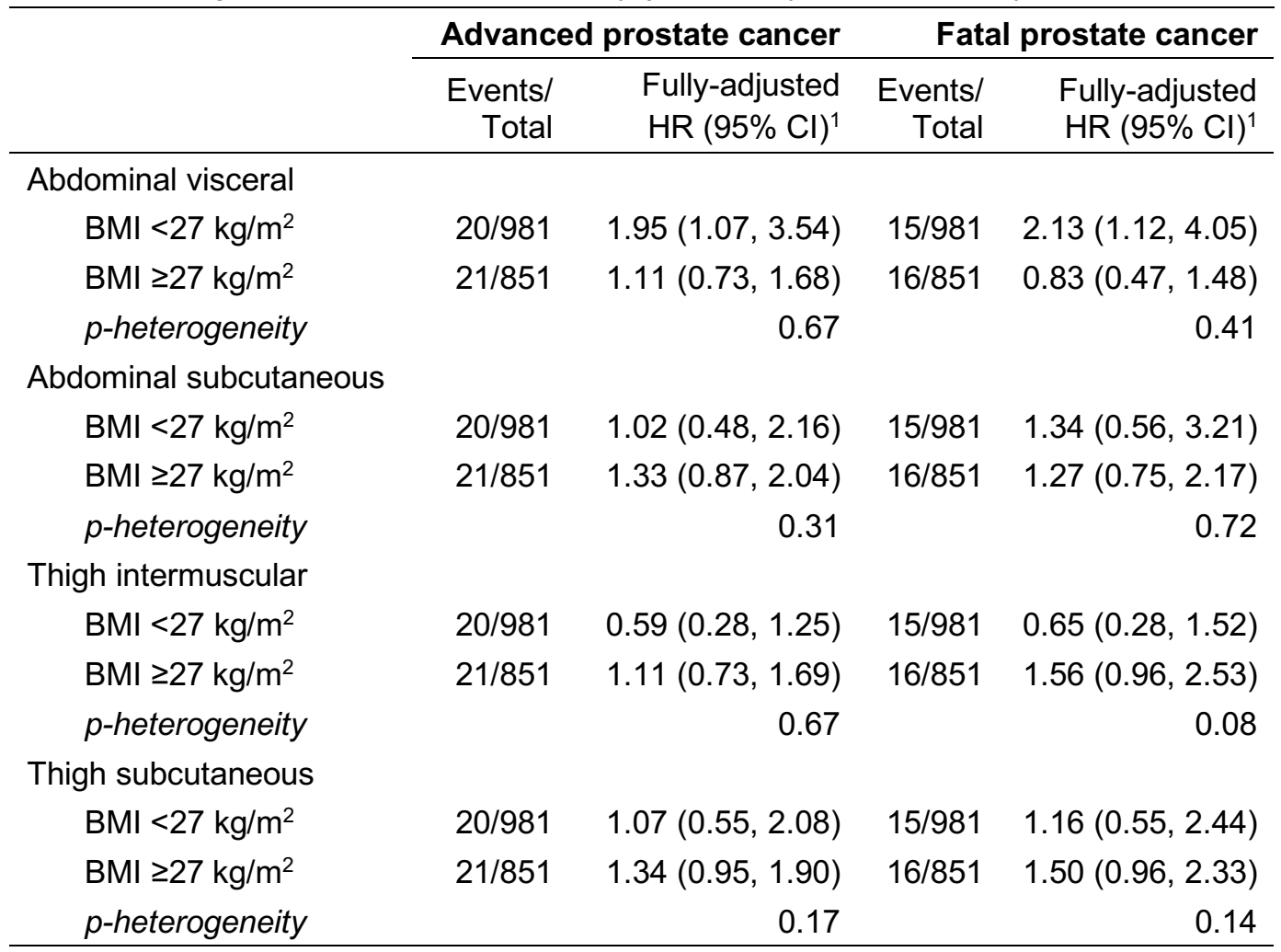

HR: Hazard ratio; Cl: Confidence interval; BMI: Body mass index.

Continuous adiposity measures were modeled per 1 standard deviation (SD) increase: abdominal visceral fat $\left(85.7 \mathrm{~cm}^{2}\right)$, abdominal subcutaneous fat $\left(85.6 \mathrm{~cm}^{2}\right)$, thigh intermuscular $\left(16.0 \mathrm{~cm}^{2}\right)$, thigh subcutaneous $\left(39.2 \mathrm{~cm}^{2}\right)$.

${ }^{1}$ Adjusted for the following variables, measured at study entry: age (continuous), height (continuous), family history of prostate cancer (yes, no), smoking status (never, former, current), education (primary/secondary, college/university), physical activity ( $\leq 3$ hours/week, $\geq 4$ hours/week), and physician visit over past 12 months (yes, no). 


\section{DISCUSSION}

In this prospective cohort of Icelandic men with objective measures of adiposity, visceral and thigh subcutaneous adiposity were the fat depots most strongly associated with risk of advanced and fatal prostate cancer, respectively. Among men with a lower BMI, visceral adiposity was the fat depot most strongly associated with both advanced and fatal prostate cancer. BMI and waist circumference were also associated with a higher risk of advanced and fatal prostate cancer. No adiposity measures were associated with total or high-grade prostate cancer.

To our knowledge, this is the first prospective study of directly measured fat distribution and risk of advanced prostate cancer. Previous retrospective and crosssectional studies incorporating CT measures of adiposity have reported mixed findings. ${ }^{4,5,18} \mathrm{~A}$ case-control study reported a positive association between visceral fat and total prostate cancer. ${ }^{4}$ In contrast, we found an association between prospectively measured visceral fat and risk of advanced and fatal prostate cancer, but not total prostate cancer. In cross-sectional studies of men undergoing radiotherapy for prostate cancer, higher visceral and abdominal subcutaneous fat were associated with higher National Comprehensive Cancer Network prostate cancer risk group, ${ }^{18}$ and abdominal subcutaneous adiposity was also associated with high-grade prostate cancer. ${ }^{5}$ One of these studies found that visceral fat and high-grade (Gleason grade $\geq 7$ ) prostate cancer

were positively associated among black men but not associated among non-black men, similar to our results in a population of white men. ${ }^{5}$ These previous studies differed in design, size, participant characteristics (e.g. age, race, adiposity measures), modeling of adiposity measures, and analytic approach. 
BMI has been associated with a higher risk of advanced and fatal, but not total, prostate cancer, ${ }^{3}$ which is in agreement with our findings. A meta-analysis showed an $8 \%$ higher risk of advanced prostate cancer (RR 1.08, 95\% Cl: $1.04-1.12 ; 23$ studies) and $11 \%$ higher risk of prostate cancer-specific mortality (RR 1.11, 95\% $\mathrm{Cl}: 1.06-1.17$; 12 studies) per $5 \mathrm{~kg} / \mathrm{m}^{2}$ increase in BMI. ${ }^{3}$ In the present study, we found that each 5 $\mathrm{kg} / \mathrm{m}^{2}$ increase in BMI was associated with a $50 \%$ higher risk of advanced and fatal prostate cancer. Different estimates across studies may be related to the timing of BMI measurement, length of follow-up, and patient characteristics. For example, studies suggest that the association between $\mathrm{BMI}$ and prostate cancer risk may differ according to age ${ }^{19,20}$ and race. ${ }^{21}$ Because age and race are key determinants of fat distribution, ${ }^{22}$ the heterogeneity of findings for BMI may be partly explained by variation in fat distribution patterns differentially associated with prostate cancer.

Findings for waist circumference, a surrogate of central adiposity, and prostate cancer have been mixed. Some studies have found higher waist circumference to be associated with a higher risk of advanced and high-grade prostate cancer, ${ }^{2,3}$ while other studies have been null. ${ }^{23}$ This is in line with our findings for a positive association between waist circumference and advanced prostate cancer, but null results for highgrade prostate cancer. Waist circumference is limited by the inability to differentiate visceral from subcutaneous adipose, which may partly explain heterogeneous findings.

Percent body fat, measured using bioelectric impedance, has been associated with high-grade prostate cancer in case-control studies..$^{24,25}$ In contrast, we found no association between prospectively measured percent body fat and high-grade prostate cancer. A prospective analysis of 10,564 initially cancer-free men in the Malmö Diet and 
Cancer cohort similarly found no association between percent body fat and risk of aggressive prostate cancer ( $\geq \mathrm{cT} 3$ or $\mathrm{N} 1$ or $\mathrm{M} 1$, or Gleason $\geq 8$, or pre-treatment PSA $\geq 50 \mathrm{ng} / \mathrm{mL})^{23}$

A prospective study among 129,502 men in the European Prospective Investigation into Cancer and Nutrition (EPIC) reported that central adiposity, assessed by waist circumference, was associated with a higher risk of advanced and high-grade prostate cancer, particularly among men with a healthy $\mathrm{BMI}^{2}$ We similarly found that the association between visceral adiposity and advanced prostate cancer was stronger among men with a lower versus higher BMI, though confidence intervals were wide. Further exploration of metabolically unhealthy, normal weight phenotypes with respect to prostate cancer outcomes is needed.

Fat distribution may be an important prognostic factor for prostate cancer outcomes by serving as a marker for metabolic, hormonal, and inflammatory milieus that play a role in prostate carcinogenesis. ${ }^{3,6-13}$ For example, visceral fat is inversely associated with bioavailable testosterone ${ }^{7,8}$ and adiponectin, ${ }^{4}$ and more strongly associated with insulin resistance and pro-inflammatory cytokines than subcutaneous fat $^{13}$ - factors that may influence prostate cancer progression. ${ }^{12,13,26,27}$

Further studies are needed to investigate whether the fat depots themselves exert systemic or local effects in ways that promote aggressive disease, or whether they are markers for a physical activity pattern or underlying hormonal milieu that influences both fat distribution and aggressive disease ${ }^{28}$ For example, fat may be preferentially deposited in the visceral depot among leaner men in the presence of a particular 
hormonal milieu. If this hormonal milieu is also a prognostic factor for advanced prostate cancer, this could partially explain the results of our analyses stratified by BMI.

These findings should be considered in light of potential limitations and strengths. Exposures were measured once at cohort entry, so we were unable to assess changes in fat depots over time. However, given the follow-up time, we were able to assess adiposity in a reasonable etiologic time window of exposure. ${ }^{29,30}$ It has been hypothesized that obese men may experience delayed detection (due to lower PSA values and biopsy accuracy) and therefore more advanced disease at diagnosis than leaner men, which might partially explain our findings of a higher risk of aggressive prostate cancer for men with higher overall obesity. ${ }^{31,32}$ However, we found that higher visceral fat was associated with a higher risk of aggressive disease even among leaner men based on BMI. We cannot rule out the possibility that our findings might be partially explained by differences in PSA testing, although our population was not subject to routine PSA testing and we adjusted for a measure of recent healthcare utilization to account for varying degrees of diagnosis opportunity. The number of advanced and fatal cancers was small and thus power was reduced. Lastly, our study population consisted of older white men, so results may not be generalizable to younger, more diverse groups of men.

The major strength of this study is that it is the first prospective analysis of CT quantified fat depots and prostate cancer risk. Our prospective design minimizes the likelihood of reverse causation, whereby the disease or its treatment influences fat distribution. Further, the use of gold-standard measures of fat distribution enabled us to examine the obesity-prostate cancer link with higher resolution than studies of BMI and 
waist circumference. This provides more insight into potential underlying mechanisms. The misclassification of fat distribution is a risk in studies relying on surrogate measures and may contribute to the variability in epidemiologic findings on obesity and prostate cancer. Precise measures of fat distribution are particularly important among older individuals, because BMI becomes a less reliable measure of adiposity with age due to the loss of lean body mass and redistribution of adipose toward the visceral compartment. ${ }^{33}$ Additional strengths of this study include its population-based sample, long duration of follow-up, complete and reliable outcome data obtained through registry linkage, and the availability of comprehensive questionnaire data.

In summary, our findings suggest that visceral and thigh subcutaneous adiposity are the most important fat depots for risk of advanced and fatal prostate cancer, and, further, that visceral adiposity is the most important depot for these outcomes among leaner men based on BMI. Studies of BMI or waist circumference alone may not capture these important sub-phenotypes, which may explain the heterogeneity of previous findings for obesity and prostate cancer. Further studies are needed to prospectively investigate fat distribution and prostate cancer outcomes, with attention to changes in fat depots over time, biological pathways, and potential heterogeneity by BMI. The identification of the adiposity phenotypes at highest risk of aggressive prostate cancer may help elucidate the mechanisms linking obesity with aggressive disease and target intervention strategies. 


\section{Guideline-based physical activity and survival} among US men with nonmetastatic prostate cancer

Barbra A. Dickerman, Edward Giovannucci, Claire H. Pernar, Lorelei A. Mucci, Miguel A. Hernán 


\section{ABSTRACT}

Purpose: In the absence of mortality data from randomized trials of prostate cancer patients, we emulated a target trial of guideline-based physical activity interventions and 10-year survival among US men with nonmetastatic prostate cancer.

Methods: We used observational data on 2,299 men in the Health Professionals Follow-up Study diagnosed with nonmetastatic prostate cancer from 1998-2010 and free of conditions that might preclude participation at baseline (defined as first postdiagnostic questionnaire). We estimated their survival under several guideline-based physical activity interventions starting at baseline and ending at the development of metastasis or conditions limiting physical ability. Information on physical activity and risk factors was updated every two years. We adjusted for baseline and time-varying risk factors for death using the parametric g-formula.

Results: We observed 250 deaths within 10 years of diagnosis. Compared with the observed $15.4 \%$ mortality risk, the estimated 10 -year risk ( $95 \%$ confidence interval, $\mathrm{Cl}$ ) of mortality was $13.0 \%(10.9-15.4 \%)$ and $11.1 \%(8.7-14.1 \%)$ for $\geq 1.25$ and $\geq 2.5$ hours/week of vigorous activity, and $13.9 \%$ (12.0-16.0\%) and $12.6 \%(10.6-14.7 \%)$ for $\geq 2.5$ and $\geq 5$ hours/week of moderate activity, respectively. Under strategies restricted to men free of chronic disease, the corresponding estimates were $14.2 \%(12.4-16.2 \%)$, $13.1 \%(11.2-15.3 \%), 14.3 \%(12.7-16.4 \%)$, and $13.7 \%(11.9-15.6 \%)$.

Conclusion: We estimated that these US men with nonmetastatic prostate cancer would have experienced clinically meaningful reductions in mortality had they all followed current physical activity recommendations until the development of conditions 
limiting physical ability. These findings may help guide clinical recommendations for prostate cancer patients and future trial design.

\section{INTRODUCTION}

Observational studies suggest that physical activity after prostate cancer diagnosis may decrease the risk of progression and mortality. ${ }^{1-6}$ Short-term randomized trials among prostate cancer survivors have demonstrated that physical activity interventions are feasible and improve body composition, biomarkers of cardiovascular health, and quality of life. ${ }^{7-9}$ To date, randomized trials have not reported on survival outcomes. To generate evidence supporting the incorporation of physical activity programs into routine cancer care, the American Society of Clinical Oncology recently called for large-scale trials of cancer survivors to evaluate the effect of physical activity interventions on outcomes such as survival. ${ }^{10}$

The feasibility of a randomized trial of physical activity and survival among men with nonmetastatic prostate cancer is limited by the long follow-up that would be required for this slowly-progressing disease. In the absence of data from such a trial, this effect needs to be estimated from observational studies. ${ }^{11}$ Previous observational studies of post-diagnosis physical activity and survival that did not adjust for prediagnosis activity have reported associations that may partially reflect the effect of lifelong physical activity habits rather than post-diagnosis changes in physical activity. Further, these studies did not report adjusted measures of absolute mortality and population attributable risks under realistic physical activity strategies. Therefore, the 
clinical impact of adhering to current guidelines for physical activity after prostate cancer diagnosis is unknown.

Here we evaluate the effects on mortality of several physical activity interventions initiated at the time of prostate cancer diagnosis. To do so, we emulate a target trial of sustained, guideline-based physical activity interventions in men with nonmetastatic prostate cancer. ${ }^{12}$ We applied the parametric g-formula, a generalization of standardization to time-varying exposures and confounding, to estimate the 10-year risk of all-cause mortality under several possible interventions.

\section{MATERIALS AND METHODS}

\section{Study data}

We leveraged data from the prospective Health Professionals Follow-up Study (HPFS), an ongoing cancer epidemiology cohort of 51,529 US male health professionals aged 40-75 years at enrollment in 1986. Participants have reported detailed clinical and lifestyle information at enrollment and every two years thereafter.

Physical activity has been reported every two years since baseline using a validated questionnaire on type, frequency, and intensity of each activity. ${ }^{13}$ Vigorous activity was defined as activity requiring $\geq 6.0$ metabolic equivalent of task (MET) scores (running, jogging, bicycling, lap swimming, tennis, squash/racquetball, calisthenics/rowing, other aerobics). Moderate activity was defined as activity requiring 3.0-5.9 METs (walking, digging, moderate-to-heavy outdoor activity, weight lifting, lowintensity exercise). Men reported the average number of weekly hours spent at each activity in the prior year. For reports that were a range, the midpoint was used as a 
measure of weekly time spent in that activity. Time spent in activities was summed to obtain total weekly duration of physical activity. In a validation study in HPFS, correlations between questionnaire- and diary-based activity scores were +0.58 for vigorous activity. ${ }^{13}$ We truncated physical activity values at the $99^{\text {th }}$ percentile to prevent implausible values from affecting our analyses.

Height and parental history of myocardial infarction before age 60 were ascertained in 1986. Biennial questionnaires have been mailed since then to update information on weight, cigarette smoking, functional impairment, and chronic diseases. A previous validation study in HPFS showed that self-reported and technician-measured weights were highly correlated (Pearson $r=0.97) .{ }^{14}$ Body mass index (BMI) was calculated as weight $(\mathrm{kg}) /$ height $(\mathrm{m})^{2}$.

Men were asked on the biennial questionnaires whether prostate cancer had been diagnosed in the prior two years. We verified the diagnosis (ICD-9: 185) using medical records and pathology reports from treating physicians and hospitals. In a standardized review, we abstracted information on diagnosis date, clinical stage, Gleason grade, prostate-specific antigen (PSA) levels, and initial treatment. We obtained information on subsequent metastasis development from prostate cancerspecific biennial questionnaires sent to all prostate cancer survivors and their physicians. 


\section{Target trial emulation}

We outlined the protocol of a target trial of physical activity interventions among men with nonmetastatic prostate cancer (Appendix Table 2.1), and emulated this trial using data from the HPFS.

\section{Eligibility criteria}

Eligible men for the current study were diagnosed with nonmetastatic prostate cancer (T1-4/N0-1/M0) between 1998 and 2010. At baseline (the first post-diagnostic questionnaire), we excluded men older than 80 years and who had developed metastasis since diagnosis, had a recent cardiovascular event (myocardial infarction or stroke), congestive heart failure, amyotrophic lateral sclerosis, or functional impairment (difficulty climbing a flight of stairs or walking eight blocks due to a physical impairment), under the assumption that a physical activity intervention would not be feasible among these men.

We also excluded men who did not have information on physical activity, BMI, smoking history, primary treatment, clinical stage, Gleason grade, or PSA level at diagnosis. After these exclusions, 2,299 men were available for the analysis. Figure 1.1 shows a flowchart of participant selection. 


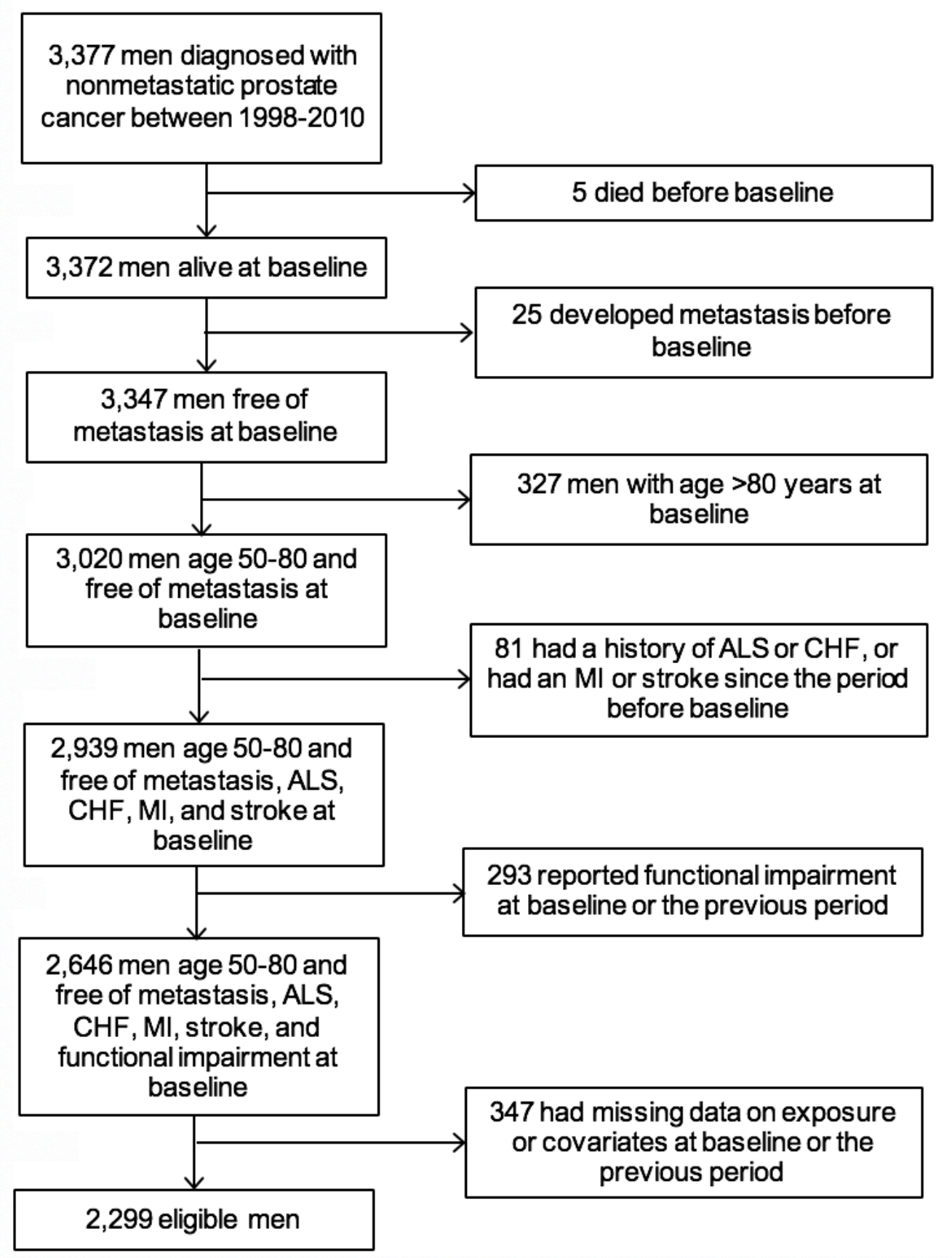

Figure 1.1 Flowchart for selection of study participants from the Health Professionals Follow-up Study. Baseline refers to the return date of the first questionnaire after prostate cancer diagnosis. ALS, amyotrophic lateral sclerosis; CHF, congestive heart failure; MI, myocardial infarction. 


\section{Outcome and follow-up}

Our outcome of interest was all-cause mortality within 10 years of diagnosis.

Deaths were ascertained through repeated mailings, telephone calls to nonrespondents, and searches of the National Death Index. We followed men from baseline (the date of first post-diagnostic questionnaire return) until death, incomplete follow-up (questionnaire non-response), 10 years after baseline, or administrative end of follow-up in June 2014, whichever happened first.

\section{Physical activity strategies}

We considered dynamic strategies of vigorous activity: at least (1) 1.25 hours/week, (2) 2.5 hours/week, (3) 3.75 hours/week, and of moderate activity: at least (4) 2.5 hours/week, (5) 5 hours/week, (6) 7.5 hours/week, initiated at baseline and continued over follow-up until the development of conditions that may limit physical ability (functional impairment, metastasis, myocardial infarction, stroke, congestive heart failure, or amyotrophic lateral sclerosis). These are examples of threshold interventions on the natural value of treatment. ${ }^{15}$

The durations and intensities of these interventions were selected to match current guidelines ${ }^{7,16}$ for physical activity among cancer survivors: engaging in strategy 1 or 4 at a minimum, and in strategy 2 or 5 for additional health benefits. We explored higher levels of physical activity through strategies 3 and 6 .

We defined activity in units of hours/week rather than MET-hours/week for consistency with the guidelines, practicality for trial implementation, and interpretability for patients. Also, we assumed that all types of activity resulting in the recommended 
intensity and duration will produce similar effects. ${ }^{17,18}$

\section{Statistical analysis}

We used the parametric g-formula, ${ }^{19}$ a generalization of standardization to timevarying exposures and confounders, to estimate the 10-year risk of death under each physical activity strategy. The g-formula appropriately handles treatment-confounder feedback that occurs when the measured time-varying confounders are affected by prior physical activity and, under the assumptions of no unmeasured confounding and no model misspecification, validly estimates the risk had all eligible individuals adhered to the specified strategies. ${ }^{20}$ The parametric g-formula has been previously applied to estimate the effect of lifestyle interventions on risk of coronary heart disease, ${ }^{21-23}$ type 2 diabetes,$^{24}$ stroke,$^{25}$ and asthma. ${ }^{26}$

We adjusted for the baseline covariates: age, parental history of myocardial infarction before age 60, primary treatment, clinical stage, Gleason grade, and PSA level at diagnosis, and pre-baseline values of BMI, smoking history, and vigorous and moderate physical activity; and the time-varying covariates: vigorous and moderate physical activity, BMI, and the development of conditions excluded at baseline (functional impairment, metastasis, myocardial infarction, stroke, congestive heart failure, and amyotrophic lateral sclerosis). All models included indicator variables for period of follow-up, baseline covariates, and time-varying covariates measured at the previous questionnaire. See Appendix 2.2 and Supplemental Material for details on the g-formula, covariates, and models. 
We compared the estimated 10-year risks had all eligible men followed each of the strategies with the risk under the observed levels of physical activity, i.e., no intervention or "natural course", via a risk ratio and a risk difference. The population attributable risk is one minus this risk ratio. We used nonparametric bootstrapping with 500 samples to obtain percentile-based $95 \%$ confidence intervals. We also generated adjusted survival curves and estimated the amount of survival time gained and time invested in physical activity over 10 years under each strategy.

\section{Sensitivity analyses for unmeasured confounding}

A key threat for the validity of our estimates is potential confounding by cancer severity or chronic disease if these conditions are severe enough to affect both physical activity and risk of death. The g-formula provides a natural way to partly address this problem by estimating the risk under physical activity interventions that are only applied at time $t$ to those who are sufficiently healthy at time $t$. Thus, in our main analysis, we considered potential interventions under which men were excused from adhering to the recommended physical activity levels after the development of functional impairment, metastasis, or a serious condition that might limit participation (myocardial infarction, stroke, congestive heart failure, amyotrophic lateral sclerosis). In sensitivity analyses, we expanded the definition of serious condition to also include angina pectoris, pulmonary embolism, heart rhythm disturbance, diabetes, chronic renal failure, rheumatoid arthritis, gout, ulcerative colitis or Crohn's disease, emphysema, Parkinson's disease, and multiple sclerosis. 
However, the validity of this approach relies critically on the assumption that the available data contains sufficient information to identify individuals who are not healthy enough to maintain the recommended physical activity levels. ${ }^{22}$ We therefore also conducted an alternative analysis in which physical activity and covariate data are lagged by two years. ${ }^{22}$ We also used a negative outcome control to detect potential unmeasured confounding by clinical disease ${ }^{27}$ Specifically, we examined the effect of physical activity on questionnaire non-response. We selected questionnaire nonresponse as an alternative outcome not directly affected by physical activity but for which the effect of physical activity would be similarly confounded (i.e. by disease severity). If the estimated effect of physical activity on non-response were null, then it would be less likely that our findings for physical activity and death were confounded through the same pathways as this negative control.

\section{Other sensitivity analyses}

We assessed the robustness of our estimates to various analytic decisions. Specifically, we (1) altered the covariate order when modeling the joint distribution of time-varying covariates reported in the same questionnaire, (2) defined different functional forms for the covariates, (3) additionally adjusted for a baseline healthy behavior score (regular multivitamin use, routine physical examinations, and screening via rectal examination, sigmoidoscopy, or colonoscopy), (4) kept the total time devoted to vigorous and moderate physical activity constant (something not implied by the guidelines) by replacing vigorous by moderate activity, and vice versa, (5) removed the cardiovascular baseline exclusions and intervened on men who developed these 
conditions over time, and (6) repeated analyses assuming that the development of functional impairment, metastasis, and chronic diseases occurred two years before the questionnaire in which they were reported.

All analyses were conducted using SAS 9.3 (SAS Institute, Inc., Cary, North Carolina). The SAS GFORMULA macro and its documentation are available at http://www.hsph.harvard.edu/causal/software. 


\section{RESULTS}

Table 2.1 shows baseline characteristics of the 2,299 eligible men. Mean age was 69 years, mean BMI was $26 \mathrm{~kg} / \mathrm{m}^{2}$, mean vigorous activity was 1.8 hours/week, and mean moderate activity was 7.1 hours/week. Most men had clinically localized (T1/T2) and low grade tumors. Of these men, 250 died within 10 years of prostate cancer diagnosis and 147 did not complete the full follow-up.

Table 2.1 Baseline characteristics of 2299 eligible participants after diagnosis with nonmetastatic prostate cancer ${ }^{a}$, Health Professionals Follow-up Study, 1998-2010

Characteristic, mean (SD) or \%

Age (years) $68.9(6.3)$

Body mass index $\left(\mathrm{kg} / \mathrm{m}^{2}\right)$

Vigorous physical activity (hours/week), current

Moderate physical activity (hours/week), current

Caucasian

Parental history of myocardial infarction (at age $\leq 60$ )

Current smoker

History of smoking before diagnosis

PSA at diagnosis $(\mathrm{ng} / \mathrm{mL})$

$<4$

4-10

68

$\geq 10$

Clinical stage at diagnosis

T1 72

$\mathrm{T} 2$

27

$\mathrm{T} 3, \mathrm{~T} 4, \mathrm{~N} 1 / \mathrm{M} 0$

Gleason grade at diagnosis

$<7$

64

7

27

$>7$

Primary treatment

Radical prostatectomy $\quad 50$

Radiation therapy $\quad 40$

Hormones 2

$\begin{array}{ll}\text { Watchful waiting } & 7\end{array}$

Other 1

${ }^{a}$ Baseline refers to the return date of the first questionnaire after prostate cancer diagnosis. 
Table 2.2 shows the estimated 10-year risk of death under strategies that excuse men from following the recommended physical activity levels after development of functional impairment, metastasis, or a serious cardiovascular or neurological condition. Compared with the $15.4 \%$ risk under no intervention, the risk $(95 \% \mathrm{Cl})$ was $13.0 \%$ (10.9-15.4\%) and $11.1 \%(8.7-14.1 \%)$ for $\geq 1.25$ and $\geq 2.5$ hours/week of vigorous activity, and $13.9 \%(12.0-16.0 \%)$ and $12.6 \%(10.6-14.7 \%)$ for $\geq 2.5$ and $\geq 5$ hours/week of moderate activity, respectively.

Table 2.2 Estimated risk of all-cause mortality under several physical activity strategies among men with nonmetastatic prostate cancer, Health Professionals Follow-up Study. All strategies excuse men from following the recommended physical activity levels after development of functional impairment, metastasis, myocardial infarction, stroke, congestive heart failure, or amyotrophic lateral sclerosis.

\begin{tabular}{lrrrrrr}
\hline & $\begin{array}{r}\text { 10-year } \\
\text { risk (\%) }\end{array}$ & $\mathbf{9 5 \%} \mathbf{~ C l}$ & Risk ratio & $\mathbf{9 5 \%} \mathbf{C l}$ & $\begin{array}{r}\text { Risk } \\
\text { difference } \\
\text { (\%) }\end{array}$ & $\mathbf{9 5 \% ~ C l ~}$ \\
\hline $\begin{array}{l}\text { No intervention } \\
\text { Vigorous activity }\end{array}$ & 15.4 & $(13.7,17.4)$ & 1.0 & -- & 0 & -- \\
$\geq 1.25 \mathrm{~h} /$ week & 13.0 & $(10.9,15.4)$ & 0.84 & $(0.75,0.94)$ & -2.4 & $(-3.9,-0.9)$ \\
$\geq 2.5 \mathrm{~h} /$ week & 11.1 & $(8.7,14.1)$ & 0.72 & $(0.58,0.88)$ & -4.3 & $(-6.6,-1.8)$ \\
$\geq 3.75 \mathrm{~h} /$ week & 10.5 & $(8.0,13.5)$ & 0.68 & $(0.53,0.85)$ & -5.0 & $(-7.3,-2.3)$ \\
Moderate activity & & & & & & \\
$\geq 2.5$ h/week & 13.9 & $(12.0,16.0)$ & 0.90 & $(0.84,0.94)$ & -1.6 & $(-2.4,-0.9)$ \\
$\geq 5$ h/week & 12.6 & $(10.6,14.7)$ & 0.81 & $(0.73,0.88)$ & -2.9 & $(-4.2,-1.8)$ \\
$\geq 7.5$ h/week & 12.2 & $(10.3,14.4)$ & 0.79 & $(0.71,0.86)$ & -3.2 & $(-4.5,-2.1)$ \\
\hline
\end{tabular}

Estimates based on the parametric g-formula adjusted for age, parental history of myocardial infarction, primary treatment, clinical stage at diagnosis, Gleason grade at diagnosis, PSA level at diagnosis, smoking history, body mass index, vigorous and moderate physical activity, and the development of functional impairment, metastasis, myocardial infarction, stroke, congestive heart failure, or amyotrophic lateral sclerosis.

a The observed risk was $15.4 \%$. There were 250 observed deaths among 2,299 men over 8,972 person-years of follow-up. Risk under no intervention is the referent. 
Figure 1.2 shows the estimated survival curves under these strategies. We estimated an average survival $1.3,2.4,0.9$, and 1.7 months longer for $\geq 1.25$ and $\geq 2.5$ hours/week of vigorous, and $\geq 2.5$ and $\geq 5$ hours/week of moderate activity, respectively, compared with no intervention (Appendix Table 2.3.1). These averages should be interpreted in the context that most men did not die over follow-up. We estimated that an additional $0.7,1.5,0.5$, and 1.2 months of physical activity were invested in the respective strategies over 10 years, compared with no intervention (Appendix Table 2.3.1).
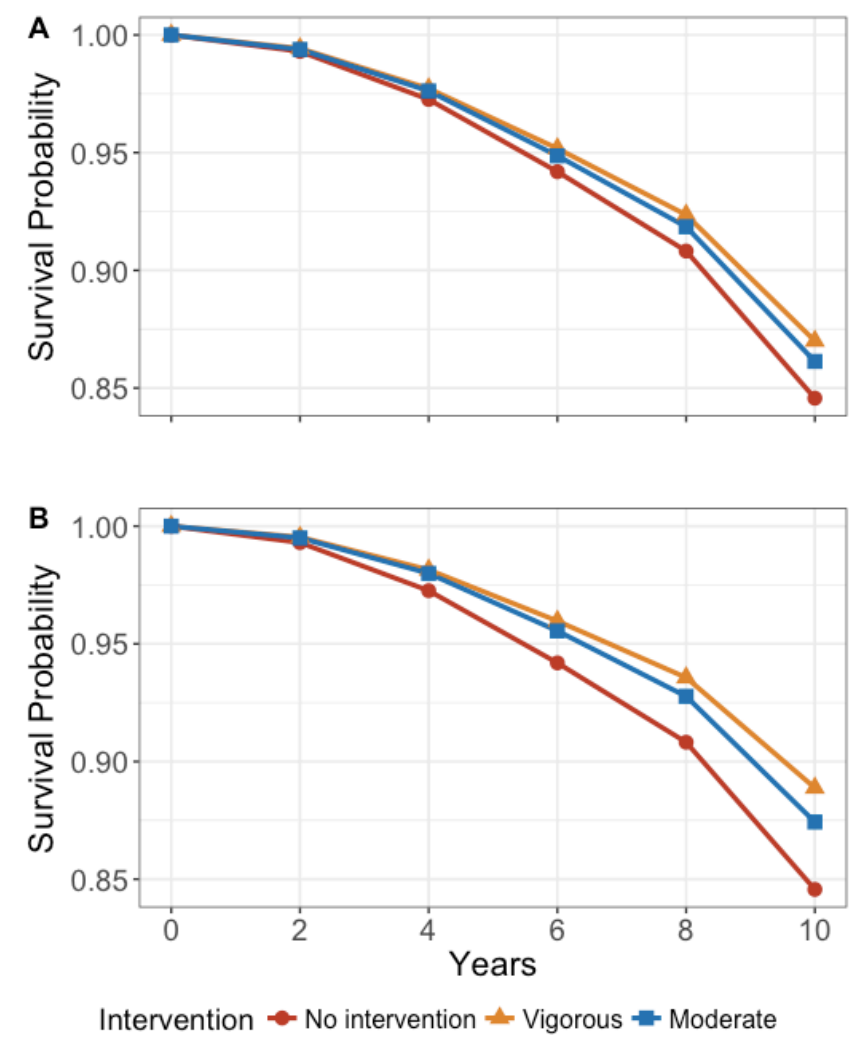

Figure 1.2 Estimated survival curves under physical activity strategies among men with nonmetastatic prostate cancer, Health Professionals Follow-up Study. All strategies excuse men from following the recommended physical activity levels after development of functional impairment, metastasis, myocardial infarction, stroke, congestive heart failure, or amyotrophic lateral sclerosis. Panel A: At least 1.25 hours/week vigorous or at least 2.5 hours/week moderate activity. Panel B: At least 2.5 hours/week vigorous or at least 5 hours/week moderate activity. 


\section{Expanding the set of chronic conditions that would excuse men from following}

the recommended physical activity levels attenuated the differences across

interventions, although risk was still lower with increasing duration and intensity of

physical activity (Table 2.3).

Table 2.3 Estimated risk of all-cause mortality under several physical activity strategies among men with nonmetastatic prostate cancer, Health Professionals Follow-up Study. All strategies excuse men from following the recommended physical activity levels after development of functional impairment, metastasis, or a serious chronic condition. ${ }^{a}$

\begin{tabular}{lrrrrrr}
\hline & $\begin{array}{r}\text { 10-year } \\
\text { risk (\%) }\end{array}$ & $\mathbf{9 5 \%} \mathbf{~ C l}$ & Risk ratio & $\mathbf{9 5 \%} \mathbf{~ C l}$ & $\begin{array}{r}\text { Risk } \\
\text { difference } \\
\text { Strategy }\end{array}$ & $\mathbf{9 5 \% ~ C l ~}$ \\
\hline $\begin{array}{l}\text { No intervention } \\
\text { Vigorous activity }\end{array}$ & 15.5 & $(13.8,17.4)$ & 1.0 & -- & 0 & - \\
$\geq 1.25$ h/week & 14.2 & $(12.4,16.2)$ & 0.92 & $(0.85,0.97)$ & -1.3 & $(-2.3,-0.5)$ \\
$\geq 2.5$ h/week & 13.1 & $(11.2,15.3)$ & 0.84 & $(0.75,0.93)$ & -2.4 & $(-3.9,-1.0)$ \\
$\geq 3.75$ h/week & 12.8 & $(10.9,14.9)$ & 0.83 & $(0.72,0.92)$ & -2.7 & $(-4.4,-1.2)$ \\
Moderate activity & & & & & & \\
$\geq 2.5$ h/week & 14.3 & $(12.7,16.4)$ & 0.93 & $(0.89,0.96)$ & -1.1 & $(-1.6,-0.6)$ \\
$\geq 5$ h/week & 13.7 & $(11.9,15.6)$ & 0.89 & $(0.83,0.92)$ & -1.8 & $(-2.7,-1.2)$ \\
$\geq 7.5$ h/week & 13.4 & $(11.8,15.5)$ & 0.87 & $(0.81,0.91)$ & -2.1 & $(-2.9,-1.3)$ \\
\hline
\end{tabular}

Estimates based on the parametric g-formula adjusted for age, parental history of myocardial infarction, primary treatment, clinical stage at diagnosis, Gleason grade at diagnosis, PSA level at diagnosis, smoking history, body mass index, vigorous and moderate physical activity, and the development of functional impairment, metastasis, myocardial infarction, stroke, congestive heart failure, or amyotrophic lateral sclerosis.

${ }^{a}$ Myocardial infarction, stroke, congestive heart failure, amyotrophic lateral sclerosis, or any of the following conditions: angina pectoris, pulmonary embolism, heart rhythm disturbance, diabetes, chronic renal failure, rheumatoid arthritis, gout, ulcerative colitis or Crohn's disease, emphysema, Parkinson's disease, and multiple sclerosis.

b The observed risk was $15.4 \%$. There were 250 observed deaths among 2,299 men over 8,972 person-years of follow-up. Risk under no intervention is the referent.

The estimated risk ratio of questionnaire non-response, our negative outcome control, was close to null (Appendix Table 2.4.1). Estimates were similar when we altered the order in which we modeled time-varying covariates reported in the same questionnaire, defined different functional forms for covariates, additionally adjusted for a baseline healthy behavior score, and included those with a recent cardiovascular event or congestive heart failure in our study population (data not shown). Estimates 
were also similar when we assumed that the development of functional impairment, metastasis, and chronic diseases occurred two years before the questionnaire in which these conditions were reported (Appendix Table 2.4.2). Risk ratio estimates were attenuated in analyses that kept the total time devoted to vigorous and moderate physical activity constant and that imposed a two-year lag (Appendix Table 2.4.3, 2.4.4; RR 0.87 and 0.85 , respectively, for 1.25 hours/week of vigorous activity, compared with 0.84 in Table 2.2; RR 0.93 and 0.95, respectively, for 2.5 hours/week of moderate activity, compared with 0.90 in Table 2.2). 


\section{DISCUSSION}

In the US, there are an estimated 160,000 men diagnosed with prostate cancer each year and 3 million survivors alive today ${ }^{28}$ Most of these men will die from causes other than their cancer. ${ }^{29}$ We used observational data to generate the first survival estimates of adhering to current physical activity guidelines after diagnosis with nonmetastatic prostate cancer until it becomes impractical. Our results suggest that 13$16 \%$ of deaths in our population would have been prevented over a 10 -year period had all men performed $\geq 1.25$ hours/week of vigorous activity and $5-10 \%$ of deaths would have been prevented had they performed $\geq 2.5$ hours/week of moderate activity until the development of metastasis or conditions limiting physical ability. Survival benefits appeared to increase for double these durations but plateaued for durations beyond that. All estimates reflect survival benefits during the 10 -year study period but not potential future benefits beyond it.

No randomized trial has directly evaluated the effect of physical activity on 10year survival among men with nonmetastatic prostate cancer. Short-term trials among prostate cancer survivors have demonstrated that physical activity interventions are feasible and improve body composition, cardiovascular biomarkers, and quality of life, ${ }^{7-9}$ but our survival curves suggest that longer trials are needed to detect survival differences as it may take two to four years for differences to emerge across strategies. The ongoing INTERVAL trial ${ }^{30}$ of men with metastatic castrate-resistant prostate cancer aims to evaluate 3-year survival but has not yet reported results.

Observational studies have compared relative rates of death among prostate cancer survivors reporting different levels of post-diagnosis physical activity. ${ }^{1,3-5}$ Three 
studies of US and Swedish men included those with nonmetastatic or localized prostate cancer, ${ }^{1,3,4}$ and one study of Canadian men also included those with metastatic disease. ${ }^{5}$ Physical activity exposure has been defined as total activity, ${ }^{1,3-5}$ walking or biking, ${ }^{3,4}$ household work, ${ }^{3}$ "exercising," ${ }^{3}$ vigorous activity, ${ }^{1,5}$ or nonvigorous activity, ${ }^{1}$ in units of MET-h/week or time/week. Two studies adjusted for pre-diagnosis activity, ${ }^{1,5}$ and two did not. .,4 $^{3,}$ wo presented unadjusted Kaplan-Meier curves..$^{3,5}$

Of the studies with an exposure definition most comparable to ours, the Prostate Cohort Study reported an all-cause mortality hazard ratio of $0.65(95 \% \mathrm{Cl}: 0.46-0.92)$ for $>3.5$ vs. 0 hours/week of post-diagnosis vigorous activity in Canadian men, which is consistent with our findings for a similar contrast. ${ }^{5} \mathrm{~A}$ previous analysis in the HPFS reported an all-cause mortality hazard ratio of $0.51(95 \% \mathrm{Cl}: 0.36-0.72)$ for $\geq 3$ vs. $<1$ hour/week of post-diagnosis vigorous activity, after adjustment for mortality risk factors and pre-diagnosis physical activity. ${ }^{1}$ This is similar to our point estimate from conventional pooled logistic models, but more protective than our g-formula estimate (RR 0.66) comparing realistic strategies in which men follow the guidelines until the development of conditions limiting physical ability (Appendix Table 2.3.2). Also, unlike previous analyses, our approach allows us to report adjusted estimates of absolute risk and population attributable risk for physical activity that is sustained until impractical.

Our novel approach to comparative effectiveness research for cancer care has several additional strengths. We designed our study by explicitly specifying the protocol of the target trial and its observational emulation, an approach promoted in observational studies to reduce risk of bias and improve data interpretation. ${ }^{12} \mathrm{We}$ analyzed our study by applying the parametric g-formula to high-quality observational 
data, allowing us to estimate clinically-meaningful absolute risks under long-term strategies that realistically depend on evolving risk factors. Lastly, we performed several analyses to address potential reverse causation that leveraged stopping criteria as a natural way to address confounding by clinical disease as well as negative outcome controls as a tool for detecting bias in observational studies. ${ }^{27}$

As with all analyses of observational data, the validity of our estimates relies on assumptions of no unmeasured confounding, measurement error, or model misspecification. Though we adjusted for many common causes of physical activity and death, the possibility of unmeasured or residual confounding by disease severity cannot be excluded. However, evidence for a protective effect of physical activity was robust to sensitivity analyses for reverse causation. We relied on self-reported information, which is subject to measurement error. However, previous validation studies in HPFS have shown that self-reported and technician-measured weight are highly correlated (Pearson $r=0.97$ ) and that the physical activity questionnaire is reproducible and provides a useful measure of average weekly activity. ${ }^{13,14}$ Finally, our estimates may not be generalizable to other populations with different distributions of risk factors or effect modifiers.

In summary, we estimated that these US men with nonmetastatic prostate cancer would have experienced clinically meaningful reductions in mortality had they all followed current physical activity recommendations until the development of conditions limiting physical ability. These findings may help guide clinical recommendations for prostate cancer patients and future trial design. 
A metabolomics analysis of adiposity and advanced prostate cancer risk in the Health Professionals Follow-up Study

Barbra A. Dickerman, Edward Giovannucci, Ericka M. Ebot, Lorelei A. Mucci 


\section{ABSTRACT}

Background: Obesity is associated with a higher risk of advanced prostate cancer, but those with the same measured body mass index (BMI) may differ in their underlying metabolic health. We aimed to identify metabolites associated with various adiposity measures and evaluate the association between metabolically-defined obesity and risk of advanced prostate cancer.

Methods: We leveraged existing, pre-diagnostic metabolomics data from nested casecontrol studies of multiple outcomes among men in the Health Professionals Follow-up Study (HPFS). Among 236 controls, we identified adiposity-associated metabolites by calculating partial Pearson correlations between 198 metabolites and several adiposity measures: BMI, waist circumference, and derived fat mass from validated prediction equations. Lasso regression was applied to further select metabolites for prediction models of adiposity measures. Lasso prediction models were used to calculate metabolic scores representing metabolic obesity. In an independent set of 212 advanced prostate cancer cases (T3b/T4/N1/M1 or lethal during follow-up) and 212 matched controls, we used logistic regression to evaluate the association between adiposity measures and metabolic scores with risk of advanced prostate cancer. We also estimated risk for men cross-classified by their adiposity measures and metabolic scores. Lastly, we conducted stratified analyses by time until prostate cancer diagnosis. Results: All adiposity measures were associated with higher concentrations of glutamate and carnitine and lower concentrations of glutamine and glycine, in addition to alterations in various lipids. Among all men, no adiposity measure or metabolic score was associated with risk of advanced prostate cancer. Estimates for self-reported BMI 
and risk of advanced prostate cancer were higher among men closer to time of diagnosis (<5.5 years: OR for Q4 vs. Q1 of BMI 1.99, 95\% Cl: 0.88-4.58; $\geq 5.5$ years:

OR $0.60,95 \% \mathrm{Cl}: 0.26-1.37 ;$ p-heterogeneity=0.05). Men with a low waist circumference or derived fat mass but high metabolic score were at the highest risk of advanced disease.

Conclusions: BMI, waist circumference, and derived fat mass were associated with a broad range of metabolic alterations. While neither adiposity nor metabolic scores were associated with risk of advanced prostate cancer, the identified metabolites may inform future research on the mechanisms linking obesity with various disease outcomes. 


\section{INTRODUCTION}

Obesity is associated with a higher risk of advanced prostate cancer. ${ }^{1}$ However, those with the same measured body mass index (BMI) may differ in their underlying metabolic health and subsequent disease risk..$^{2-5}$ The integration of metabolomics and anthropometric data offers great potential to better identify men at highest risk, elucidate underlying mechanisms, and target intervention strategies.

Previous metabolomics-based studies have identified metabolic alterations associated with BMI and waist circumference, but not fat mass. ${ }^{4,6-10}$ Some of these studies further examined how the identified metabolites individually related to later risk of diabetes and breast cancer. ${ }^{6,8}$ No study to date has examined whether obesityrelated metabolic alterations could, collectively as a measure of metabolic obesity, facilitate the identification of men at risk of advanced prostate cancer by adding information over standard clinical markers.

Here we analyzed metabolomics data among men in the Health Professionals Follow-up Study (HPFS) to identify plasma metabolites associated with various adiposity measures (BMI, waist circumference, and derived fat mass) and use selected metabolites to evaluate the association between metabolically-defined obesity and risk of advanced prostate cancer. 


\section{METHODS}

\section{Study population}

The HPFS is an ongoing prospective cohort study of 51,529 U.S. male health professionals aged 40-75 years at enrollment in 1986. Participants have been followed every two years since enrollment to update clinical and lifestyle information and ascertain incident diseases. Blood samples were collected from 18,225 (35\%) participants from 1993-1995. Samples were mailed to our laboratory overnight on cold packs, and then centrifuged to collect and store plasma in liquid nitrogen freezers. Participants reported the timing of blood collection and fasting on a questionnaire returned with the samples.

\section{Metabolites associated with adiposity}

For the identification of adiposity-associated metabolites, we included 236 controls from nested case-control studies of amyotrophic lateral sclerosis (ALS) and Parkinson's disease (PD) in HPFS. We selected controls only to minimize the chance that latent disease influenced metabolite or adiposity measures.

\section{Metabolically-defined obesity and advanced prostate cancer risk}

To evaluate the association between metabolically-defined obesity and advanced prostate cancer risk, we included an independent set of 212 advanced prostate cancer cases $(\mathrm{T} 3 \mathrm{~b} / \mathrm{T} 4 / \mathrm{N} 1 / \mathrm{M} 1$ or lethal [distant metastasis or death due to prostate cancer] during follow-up) and 212 matched controls. Cases were all men diagnosed with advanced prostate cancer between blood draw and September 2010. For each case, 
one control was selected who was alive and cancer-free at the time of the case's diagnosis. Matching criteria were age ( \pm 1 year), recent PSA testing prior to blood draw (since January 1, 1992; yes/no), and time of day, season, and year of blood collection.

Prostate cancer diagnoses were self-reported on the biennial questionnaires and verified in a standardized review of medical records and pathology reports. We obtained information on subsequent metastasis development from prostate cancer-specific biennial questionnaires sent to all prostate cancer survivors and their physicians. Prostate cancer-specific deaths were verified through review of medical records and death certificates. Follow-up is $>96 \%$ complete for prostate cancer incidence and $>98 \%$ for mortality.

\section{Adiposity measures and covariates}

We examined BMl, waist circumference, and derived fat mass assessed in the questionnaire closest to the date of blood collection. Participants reported weight and height in 1986 and updated weight every two years thereafter. They reported waist circumference in 1987 and 1996. A previous validation study in HPFS showed that selfreported weights and waist circumferences were highly correlated with technicianmeasured values (Pearson $r=0.97$ and $r=0.95$, respectively). ${ }^{11}$ We derived fat mass using a prediction equation developed in the NHANES. ${ }^{12}$ Previous validation in NHANES showed that this equation was highly predictive of DXA-measured fat mass $\left(R^{2}=0.90\right) .{ }^{12}$ We assessed cigarette smoking, physical activity, and history of diabetes in the questionnaire before blood collection. 
We excluded participants missing data on adiposity measures for the respective analyses. We excluded one participant with a BMI $>55 \mathrm{~kg} / \mathrm{m}^{2}$ from the BMI analyses to prevent extreme values from affecting our analyses. Appendix Figure 3 shows a flowchart of participant selection.

\section{Metabolite profiling}

All included men had existing metabolomics data measured in pre-diagnostic blood samples. Polar metabolites and lipids were profiled using a combination of liquid chromatography tandem mass spectrometry (LC-MS) methods described

previously. ${ }^{13,14}$ Briefly, profiling data were acquired using a Nexera X2 U-HPLC (Shimazdu Corp.) coupled to Q Extractive hybrid quadrupole and Exactive Plus orbitrap mass spectrometers (Thermo Fisher Scientific). Polar metabolites were separated using hydrophilic interaction liquid chromatography with positive ion mode detection (HILICpos), and polar and non-polar lipids were profiled using C8 chromatography with positive ion mode detection (C8-pos). Metabolite identities were confirmed using authentic reference standards or reference samples. The nomenclature for lipid analytes included the number of carbons and number of double bonds in the lipid acyl chain. Metabolite levels were reported as measured LC-MS peak areas, which are proportional to metabolite concentration.

A pilot study in the HPFS and Nurses' Health Study assessed the reliability of this platform using our archival plasma samples as well as within-person variability. ${ }^{14}$ Most metabolites performed well, particularly lipids and lipid metabolites (coefficient of 
variation $[C V]=8 \%$, range $1-14 \%$ ). Fasting status had little influence on the CVs. Almost $90 \%$ of metabolites were stable over two years within individuals.

Among known metabolites, we excluded 89 metabolites with mean $\mathrm{CV}>25 \%$ or ICC $<0.4$ based on quality control samples embedded in each project and 12 metabolites with undetectable levels for $\geq 10 \%$ of participants. We restricted to metabolites measured across all endpoints, matching by HMDB ID. This left 198 metabolites for analysis. Appendix 3.1 includes details on metabolite selection. For metabolites with undetectable levels for $<10 \%$ of participants, we imputed half the lowest value of the metabolite if the participant's sample was missing $\leq 25 \%$ of metabolites (which applied in all cases). Metabolite peak areas were In-transformed to improve normality and then standardized (to mean $=0, S D=1$ ) within each project to facilitate analyses across projects.

\section{Statistical analysis}

Among the 236 controls in the ALS and PD studies, we identified metabolites associated with adiposity by calculating partial Pearson correlations between 198 metabolites and each adiposity measure, adjusting for age and smoking status at time of blood draw. We obtained conservative estimates of false discovery rate (FDR) via the Benjamini-Hochberg procedure. ${ }^{15}$ We carried forward metabolites with an FDR p-value $<0.05$ and $|r| \geq 0.15$. Lasso regression models were used to further select metabolites for prediction models of adiposity measures. This approach allowed us to account for the relative importance of each identified metabolite in the presence of the others and generate a parsimonious model. 
In an independent set of 212 advanced prostate cancer cases and 212 matched controls, we applied the Lasso models to predict metabolic scores given men's underlying levels of adiposity-associated metabolites. The BMI metabolic score, for example, ranks men by their expected BMI given their underlying levels of BMIassociated metabolites. These metabolic scores were not intended to be surrogates of obesity, but rather to provide information about metabolic obesity that may be relevant in the pathophysiology of disease. Their estimation was informed by the relationships between adiposity measures and metabolic profiles observed in the reference population, which are shaped by upstream lifestyle and genetic factors. A high metabolic score can be interpreted as having a metabolic profile consistent with an average man of high adiposity in the reference population.

We used logistic regression to estimate odds ratios (OR) and 95\% confidence intervals (Cls) for advanced prostate cancer for each adiposity measure and metabolic score, fit in separate models. Under this study design, the odds ratio from logistic regression estimates the rate ratio that would be obtained from a cohort design. We used unconditional logistic regression for efficiency, adjusting for age, diabetes, physical activity, smoking status, family history of prostate cancer, and recent PSA screening (a matching factor). We did not adjust for other matching factors unrelated to our exposures or outcome of interest. Additionally adjusting all models for fasting status and adjusting fat mass models for height did not change our estimates (data not shown). Further, we estimated risk for men cross-classified by their adiposity measures and metabolic scores, split at the median. Lastly, we conducted stratified analyses to evaluate whether estimates varied by median time until prostate cancer diagnosis $(<5.5$ 
vs. $\geq 5.5$ years). Controls contributed to the stratum containing their matched case. Tests for heterogeneity were performed using likelihood ratio tests comparing models with and without a product term between the exposure of interest and an indicator for median time until diagnosis.

All analyses were conducted using RStudio 1.1.423. 


\section{RESULTS}

Population characteristics

Table 3.1 shows baseline characteristics of the 660 eligible men. Mean age at blood draw was 65 years, mean BMI was $26 \mathrm{~kg} / \mathrm{m}^{2}$, mean waist circumference was 96 $\mathrm{cm}$, and mean derived fat mass was $22 \mathrm{~kg}$. Controls in the PD study were more likely to be former smokers compared with men in the ALS and prostate cancer studies.

Advanced prostate cancer cases were more likely to be current smokers than their matched controls.

Table 3.1 Baseline characteristics of participants from nested case-control studies of various outcomes, Health Professionals Follow-up Study. ${ }^{a}$

\begin{tabular}{lrrrr}
\hline & ALS study & PD study & \multicolumn{2}{r}{ Prostate cancer study } \\
\hline Characteristic, & $\begin{array}{r}\text { Controls } \\
(\mathrm{N}=52)\end{array}$ & $\begin{array}{r}\text { Controls } \\
(\mathrm{N}=184)\end{array}$ & $\begin{array}{r}\text { Controls } \\
(\mathrm{N}=212)\end{array}$ & $\begin{array}{r}\text { Advanced cases } \\
\text { mean (SD) or \% }\end{array}$ \\
\hline Age at blood draw (years) & $62.7(8.3)$ & $65.3(8.0)$ & $65.3(8.4)$ & $65.4(8.5)$ \\
Race/ethnicity, White & 94 & 98 & 97 & 99 \\
Year of blood donation & & & & \\
$\quad 1993-1994$ & 87 & 94 & 97 & 97 \\
$\quad 1995-1996$ & 13 & 6 & 3 & 3 \\
Fasting $\geq 8$ hours at blood draw & 50 & 55 & 62 & 67 \\
Body mass index (kg/m $\left.{ }^{2}\right)$ & $25.9(2.5)$ & $25.5(2.8)$ & $25.8(3.6)$ & $25.8(4.1)$ \\
Waist circumference (cm) & $94.7(8.1)$ & $95.5(8.5)$ & $96.5(10.3)$ & $95.9(10.0)$ \\
Derived fat mass (kg) & $21.9(4.3)$ & $21.6(5.2)$ & $22.5(6.4)$ & $21.9(6.0)$ \\
Total physical activity (METs/week) & $39.7(29.3)$ & $34.4(27.4)$ & $32.5(25.6)$ & $31.1(28.9)$ \\
Smoking status at blood draw & & & & \\
$\quad$ Never & 50 & 39 & 46 & 43 \\
$\quad$ Former & 46 & 58 & 50 & 51 \\
$\quad$ Current & 4 & 3 & 4 & 7 \\
History of diabetes mellitus & 4 & 4 & 6 & 7 \\
Family history of prostate cancer & -- & -- & 9 & 9 \\
Recent PSA screening & -- & -- & 60 & 62 \\
\hline ALS: Amyotr & -- & &
\end{tabular}

ALS: Amyotrophic lateral sclerosis. PD: Parkinson's disease. MET: Metabolic equivalent.

Frequencies of polytomous variables may not sum to $100 \%$ due to rounding.

a Baseline characteristics were measured in questionnaire preceding blood draw unless otherwise noted.

b Derived fat mass from validated anthropometric prediction models developed in the NHANES.

${ }^{c}$ PSA screening since January 1, 1992. 
Mean age at advanced prostate cancer diagnosis was 72 years (range, 50-92). Of the 212 advanced cases, $53 \%$ of these men had T3b/T4/N1/M1 tumors at diagnosis and the remaining $47 \%$ were classified as such because of metastasis or fatal prostate cancer over follow-up. Among the $88 \%$ of cases for whom grade was available, $33 \%$ had high-grade tumors (Gleason grade $\geq 8$ ). Among the $85 \%$ of cases for whom PSA at diagnosis was available, the median PSA was $8.5 \mathrm{ng} / \mathrm{mL}$. The median time between blood draw and prostate cancer diagnosis was 5.5 years (range, 0.1-16.2).

\section{Metabolites associated with adiposity}

Metabolite profiling resulted in 198 identified metabolites, including lipids (e.g. 12 cholesterol ester [CE], 11 diacylglycerol [DAG], 10 lysophosphatidylcholine [LPC], 6 lysophosphatidylethanolamine [LPE], 35 phosphatidylcholine [PC], 20

phosphatidylethanolamine [PE], 9 sphingomyeline [SM], 42 triacylglycerol [TAG]), amino acids, and other small molecules (Appendix 3.1).

We identified 50 metabolites associated with BMI, 43 with waist circumference, and 35 with derived fat mass (Table 3.2). There was considerable overlap in identified metabolites across adiposity measures; 30 metabolites were associated with all three measures. All adiposity measures were associated with higher concentrations carnitine, glutamate, DAG, and TAG. All were associated with lower concentrations of glycine, glutamine, CE, LPC, and LPE. Mixed associations were found for PC. BMI and waist circumference were also associated with higher SM. Waist circumference and fat mass were both associated with higher 1-methyladenosine. Results from sensitivity analyses restricted to men with data on all three adiposity measures are shown in Appendix 
Table 3. In our models to derive metabolic scores, there were 15 metabolites with a non-zero coefficient in models for BMI, 15 for waist circumference, and 27 for derived fat mass (Appendix 3.3). Each model retained the amino acids and top hit for carnitines, CE, LPC, and LPE, in addition to a selection of lipids. 


\begin{tabular}{|c|c|c|c|c|c|c|c|}
\hline \multirow[b]{2}{*}{ HMDB ID } & \multirow[b]{2}{*}{ Metabolite } & \multicolumn{2}{|c|}{ Body mass index } & \multicolumn{2}{|c|}{ Waist circumference } & \multicolumn{2}{|c|}{ Derived fat mass } \\
\hline & & $\begin{array}{c}\text { Pearson } \\
\text { correlation } \\
\text { coefficient }\end{array}$ & $\begin{array}{l}\text { FDR P- } \\
\text { value }\end{array}$ & $\begin{array}{c}\text { Pearson } \\
\text { correlation } \\
\text { coefficient }\end{array}$ & $\begin{array}{l}\text { FDR P. } \\
\text { value }\end{array}$ & $\begin{array}{r}\text { Pearson } \\
\text { correlation } \\
\text { coefficient }\end{array}$ & $\begin{array}{r}\text { FDR P- } \\
\text { value }\end{array}$ \\
\hline \multicolumn{8}{|l|}{ Amino acids } \\
\hline HMDB00148 & glutamate & 0.31 & 8.3E-05 & 0.25 & $9.0 \mathrm{E}-03$ & 0.28 & $1.8 \mathrm{E}-03$ \\
\hline HMDB00123 & glycine & -0.29 & 2.9E-04 & -0.20 & 0.02 & -0.25 & $5.3 \mathrm{E}-03$ \\
\hline HMDB00641 & glutamine & -0.19 & 0.02 & -0.19 & 0.03 & -0.19 & 0.04 \\
\hline \multicolumn{8}{|l|}{ Carnitines } \\
\hline HMDB06347 & C26 carnitine & 0.18 & 0.03 & 0.18 & 0.04 & -- & -- \\
\hline HMDB00688 & C5 carnitine & 0.18 & 0.03 & -- & -- & -- & -- \\
\hline HMDB00705 & C6 carnitine & 0.18 & 0.03 & 0.18 & 0.03 & -- & -- \\
\hline HMDB02013 & C4 carnitine & 0.16 & 0.05 & -- & -- & -- & -- \\
\hline HMDB13326 & C12:1 carnitine & -- & -- & 0.17 & 0.05 & -- & -- \\
\hline HMDB00222 & C16 carnitine & -- & -- & -- & -- & 0.18 & 0.05 \\
\hline \multicolumn{8}{|l|}{ Lipids } \\
\hline \multicolumn{8}{|l|}{$C E$} \\
\hline HMDB10375 & C22:5 CE & -0.20 & 0.02 & -0.22 & 0.01 & -0.23 & 0.01 \\
\hline HMDB00918 & C18:1 CE & -0.17 & 0.04 & -0.17 & 0.04 & -0.19 & 0.04 \\
\hline HMDB00610 & C18:2 CE & -0.17 & 0.04 & -0.18 & 0.04 & -- & -- \\
\hline HMDB06733 & C22:6 CE & -- & -- & -- & -- & -0.19 & 0.04 \\
\hline
\end{tabular}

CE, cholesterol ester; DAG, diacylglycerol; LPC, lysophosphatidylcholine; LPE, lysophosphatidylethanolamine; PC, phosphatidylcholine; PE, phosphatidylethanolamine; SM, sphingomyeline; TAG, triacylglycerol.

Estimates from partial Pearson correlation, adjusted for age at blood draw (continuous) and smoking status (ever/never). The number of men contributing to each analysis was 234 for BMI, 233 for waist circumference, 219 for derived fat mass.

Boldface indicates a metabolite with a non-zero coefficient when all metabolites associated with that adiposity measure were entered into Lasso. 


\begin{tabular}{|c|c|c|c|c|c|c|c|}
\hline \multirow[b]{2}{*}{ HMDB ID } & \multirow[b]{2}{*}{ Metabolite } & \multicolumn{2}{|c|}{ Body mass index } & \multicolumn{2}{|c|}{ Waist circumference } & \multicolumn{2}{|c|}{ Derived fat mass } \\
\hline & & $\begin{array}{c}\text { Pearson } \\
\text { correlation } \\
\text { coefficient }\end{array}$ & $\begin{array}{l}\text { FDR P- } \\
\text { value }\end{array}$ & $\begin{array}{c}\text { Pearson } \\
\text { correlation } \\
\text { coefficient }\end{array}$ & $\begin{array}{l}\text { FDR P- } \\
\text { value }\end{array}$ & $\begin{array}{c}\text { Pearson } \\
\text { correlation } \\
\text { coefficient }\end{array}$ & $\begin{array}{r}\text { FDR P- } \\
\text { value }\end{array}$ \\
\hline \multicolumn{8}{|l|}{ DAG } \\
\hline \multicolumn{8}{|l|}{ Saturated } \\
\hline HMDB07098 & C32:0 DAG & 0.23 & 7.4E-03 & 0.21 & 1.6E-02 & 0.22 & 0.01 \\
\hline \multicolumn{8}{|l|}{ Unsaturated } \\
\hline HMDB07102 & C34:1 DAG & 0.24 & 4.8E-03 & 0.24 & $9.0 \mathrm{E}-03$ & 0.24 & 7.9E-03 \\
\hline HMDB07099 & C32:1 DAG & 0.23 & $6.9 \mathrm{E}-03$ & 0.24 & $9.0 \mathrm{E}-03$ & 0.24 & 7.9E-03 \\
\hline HMDB07103 & C34:2 DAG & 0.22 & 8.1E-03 & 0.23 & $9.0 \mathrm{E}-03$ & 0.23 & 0.01 \\
\hline HMDB07132 & C34:3 DAG & 0.22 & $9.8 \mathrm{E}-03$ & 0.23 & $9.0 \mathrm{E}-03$ & 0.22 & 0.01 \\
\hline HMDB07218 & C36:2 DAG & 0.21 & 0.01 & 0.23 & $9.0 \mathrm{E}-03$ & 0.22 & 0.01 \\
\hline HMDB07216 & C36:1 DAG & 0.21 & 0.01 & 0.22 & 0.01 & 0.23 & 0.01 \\
\hline HMDB07219 & C36:3 DAG & 0.20 & 0.02 & 0.21 & 0.01 & 0.20 & 0.03 \\
\hline HMDB07248 & C36:4 DAG & 0.17 & 0.04 & 0.17 & 0.04 & -- & -- \\
\hline HMDB07199 & C38:5 DAG & 0.16 & 0.05 & -- & -- & -- & -- \\
\hline \multicolumn{8}{|l|}{$L P C$} \\
\hline HMDB10386 & C18:2 LPC & -0.34 & 1.4E-05 & -0.23 & $9.0 \mathrm{E}-03$ & -0.29 & 1.1E-03 \\
\hline HMDB10397 & C20:5 LPC & -0.34 & 1.4E-05 & -0.24 & $9.0 \mathrm{E}-03$ & -0.29 & $1.1 \mathrm{E}-03$ \\
\hline HMDB02815 & C18:1 LPC & -0.29 & 2.9E-04 & -0.21 & 0.02 & -0.26 & 5.3E-03 \\
\hline HMDB10404 & C22:6 LPC & -0.25 & $2.2 \mathrm{E}-03$ & -0.18 & 0.03 & -0.26 & 5.3E-03 \\
\hline
\end{tabular}

CE, cholesterol ester; DAG, diacylglycerol; LPC, lysophosphatidylcholine; LPE, lysophosphatidylethanolamine; PC, phosphatidylcholine; PE, phosphatidylethanolamine; SM, sphingomyeline; TAG, triacylglycerol.

Estimates from partial Pearson correlation, adjusted for age at blood draw (continuous) and smoking status (ever/never). The number of men contributing to each analysis was 234 for BMI, 233 for waist circumference, 219 for derived fat mass.

Boldface indicates a metabolite with a non-zero coefficient when all metabolites associated with that adiposity measure were entered into Lasso. 
Table 3.2 (Continued) Metabolites associated with adiposity measures (FDR p-value $<0.05$ and $|r| \geq 0.15$ ) among controls from nested case-control studies, Health Professionals Follow-up Study.

\begin{tabular}{|c|c|c|c|c|c|c|c|}
\hline \multirow[b]{2}{*}{ HMDB ID } & \multirow[b]{2}{*}{ Metabolite } & \multicolumn{2}{|c|}{ Body mass index } & \multicolumn{2}{|c|}{ Waist circumference } & \multicolumn{2}{|c|}{ Derived fat mass } \\
\hline & & $\begin{array}{c}\text { Pearson } \\
\text { correlation } \\
\text { coefficient }\end{array}$ & $\begin{array}{l}\text { FDR P- } \\
\text { value }\end{array}$ & $\begin{array}{c}\text { Pearson } \\
\text { correlation } \\
\text { coefficient }\end{array}$ & $\begin{array}{c}\text { FDR P- } \\
\text { value }\end{array}$ & $\begin{array}{c}\text { Pearson } \\
\text { correlation } \\
\text { coefficient }\end{array}$ & $\begin{array}{l}\text { FDR P } \\
\text { value }\end{array}$ \\
\hline \multicolumn{8}{|l|}{ LPE } \\
\hline HMDB11503 & C16:0 LPE & -0.29 & 2.9E-04 & -0.17 & 0.04 & -0.23 & 0.01 \\
\hline HMDB11507 & C18:2 LPE & -0.26 & 1.3E-03 & -- & -- & -- & - \\
\hline HMDB11506 & C18:1 LPE & -0.22 & 8.1E-03 & -- & -- & -0.18 & 0.05 \\
\hline HMDB11130 & C18:0 LPE & -0.20 & 0.02 & -- & -- & -- & - \\
\hline HMDB11526 & C22:6 LPE & -0.19 & 0.02 & -- & -- & -- & - \\
\hline \multicolumn{8}{|l|}{$P C$} \\
\hline HMDB08047 & C38:3 PC & 0.20 & 0.02 & 0.20 & 0.02 & -- & - \\
\hline HMDB07983 & C36:4 PC-A & -0.20 & 0.02 & -- & -- & -- & - \\
\hline HMDB08057 & C40:6 PC & 0.19 & 0.03 & 0.19 & 0.03 & -- & - \\
\hline HMDB08048 & C38:4 PC & 0.18 & 0.03 & -- & -- & -- & - \\
\hline HMDB08511 & C40:10 PC & -0.16 & 0.05 & -- & -- & -0.18 & 0.05 \\
\hline HMDB11210 & C34:2 PC plasmalogen & -0.16 & 0.05 & -- & -- & -- & - \\
\hline \multicolumn{8}{|l|}{$S M$} \\
\hline HMDB12103 & C22:0 SM & 0.21 & 0.01 & 0.18 & 0.03 & -- & - \\
\hline HMDB12102 & C20:0 SM & 0.17 & 0.05 & -- & -- & -- & - \\
\hline HMDB01348 & C18:0 SM & -- & -- & 0.18 & 0.04 & -- & - \\
\hline
\end{tabular}

CE, cholesterol ester; DAG, diacylglycerol; LPC, Iysophosphatidylcholine; LPE, lysophosphatidylethanolamine; PC, phosphatidylcholine; PE, phosphatidylethanolamine; SM, sphingomyeline; TAG, triacylglycerol.

Estimates from partial Pearson correlation, adjusted for age at blood draw (continuous) and smoking status (ever/never). The number of men contributing to each analysis was 234 for BMI, 233 for waist circumference, 219 for derived fat mass.

Boldface indicates a metabolite with a non-zero coefficient when all metabolites associated with that adiposity measure were entered into Lasso. 


\begin{tabular}{|c|c|c|c|c|c|c|c|}
\hline \multirow[b]{2}{*}{ HMDB ID } & \multirow[b]{2}{*}{ Metabolite } & \multicolumn{2}{|c|}{ Body mass index } & \multicolumn{2}{|c|}{ Waist circumference } & \multicolumn{2}{|c|}{ Derived fat mass } \\
\hline & & $\begin{array}{c}\text { Pearson } \\
\text { correlation } \\
\text { coefficient }\end{array}$ & $\begin{array}{l}\text { FDR P- } \\
\text { value }\end{array}$ & $\begin{array}{c}\text { Pearson } \\
\text { correlation } \\
\text { coefficient }\end{array}$ & $\begin{array}{c}\text { FDR P- } \\
\text { value }\end{array}$ & $\begin{array}{r}\text { Pearson } \\
\text { correlation } \\
\text { coefficient }\end{array}$ & $\begin{array}{r}\text { FDR P. } \\
\text { value }\end{array}$ \\
\hline \multicolumn{8}{|l|}{ TAG } \\
\hline \multicolumn{8}{|l|}{ Unsaturated } \\
\hline HMDB05369 & C52:2 TAG & 0.23 & $6.5 \mathrm{E}-03$ & 0.23 & $9.0 \mathrm{E}-03$ & 0.23 & 0.01 \\
\hline HMDB05360 & C50:1 TAG & 0.23 & 7.2E-03 & 0.21 & 0.02 & 0.22 & 0.01 \\
\hline HMDB05384 & C52:3 TAG & 0.22 & 8.1E-03 & 0.22 & 0.01 & 0.21 & 0.02 \\
\hline HMDB05433 & C50:3 TAG & 0.22 & $9.5 \mathrm{E}-03$ & 0.23 & $9.0 \mathrm{E}-03$ & 0.22 & 0.01 \\
\hline HMDB05377 & C50:2 TAG & 0.22 & 9.7E-03 & 0.21 & 0.01 & 0.22 & 0.01 \\
\hline HMDB05367 & C52:1 TAG & 0.20 & 0.02 & 0.20 & 0.02 & 0.21 & 0.02 \\
\hline HMDB05376 & C48:2 TAG & 0.19 & 0.02 & 0.20 & 0.02 & 0.21 & 0.02 \\
\hline HMDB05432 & C48:3 TAG & 0.18 & 0.03 & 0.20 & 0.02 & 0.20 & 0.02 \\
\hline HMDB10412 & C46:1 TAG & 0.18 & 0.03 & 0.17 & 0.04 & 0.20 & 0.03 \\
\hline HMDB05403 & C54:2 TAG & 0.18 & 0.03 & 0.20 & 0.02 & 0.20 & 0.03 \\
\hline HMDB05363 & C52:4 TAG & 0.18 & 0.03 & 0.18 & 0.03 & -- & -- \\
\hline HMDB05359 & C48:1 TAG & 0.17 & 0.04 & 0.17 & 0.05 & 0.18 & 0.05 \\
\hline HMDB10419 & C46:2 TAG & 0.17 & 0.05 & 0.17 & 0.05 & 0.19 & 0.04 \\
\hline HMDB05405 & C54:3 TAG & -- & -- & 0.18 & 0.04 & -- & -- \\
\hline HMDB05435 & C50:4 TAG & -- & -- & 0.17 & 0.04 & -- & -- \\
\hline \multicolumn{8}{|c|}{ Purine nucleosides } \\
\hline HMDB03331 & 1-methyladenosine & -- & -- & 0.18 & 0.03 & 0.18 & 0.05 \\
\hline
\end{tabular}

CE, cholesterol ester; DAG, diacylglycerol; LPC, lysophosphatidylcholine; LPE, lysophosphatidylethanolamine; PC, phosphatidylcholine; PE, phosphatidylethanolamine; SM, sphingomyeline; TAG, triacylglycerol.

Estimates from partial Pearson correlation, adjusted for age at blood draw (continuous) and smoking status (ever/never). The number of men contributing to each analysis was 234 for BMI, 233 for waist circumference, 219 for derived fat mass.

Boldface indicates a metabolite with a non-zero coefficient when all metabolites associated with that adiposity measure were entered into Lasso. 
Metabolically-defined obesity and advanced prostate cancer risk

Table 3.3 shows the estimated odds ratios for advanced prostate cancer over the study period in an independent set of men by self-reported adiposity and calculated metabolic scores. Estimates for all continuously-modeled measures were close to null, though confidence intervals were wide. No trends were noted across increasing quartiles of self-reported adiposity or metabolic scores. Estimates were similar in sensitivity analyses excluding men diagnosed in the first two years after blood draw to minimize the chance that latent disease influenced metabolite or adiposity measures (data not shown). Estimates for self-reported BMI and risk of advanced prostate cancer were higher among men closer to time of diagnosis ( $<5.5$ years: OR for Q4 vs. Q1 of BMI 1.99, 95\% Cl: 0.88-4.58; $\geq 5.5$ years: OR 0.60, 95\% Cl: 0.26-1.37; $p-$ heterogeneity=0.05) (Table 3.4).

Table 3.3 Estimated odds ratios (OR) and 95\% confidence intervals (Cls) of advanced prostate cancer by adiposity measures and metabolic scores (predicted adiposity using metabolites), Health Professionals Follow-up Study.

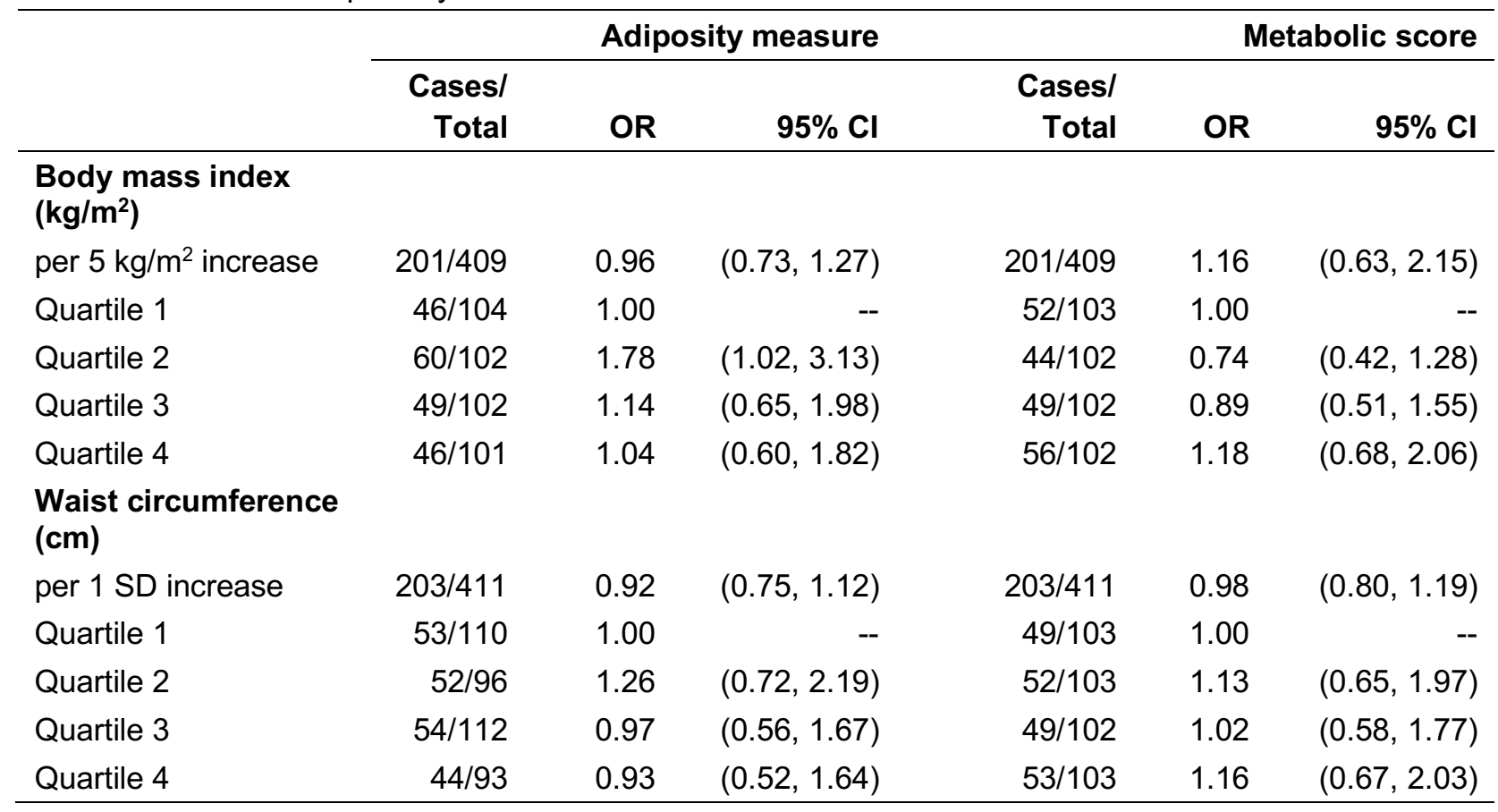


Table 3.3 (Continued) Estimated odds ratios (OR) and 95\% confidence intervals (Cls) of advanced prostate cancer by adiposity measures and metabolic scores (predicted adiposity using metabolites), Health Professionals Follow-up Study.

\begin{tabular}{lrrrrrr}
\hline & \multicolumn{3}{c}{ Adiposity measure } & \multicolumn{2}{c}{ Metabolic score } \\
\cline { 2 - 7 } & $\begin{array}{r}\text { Cases/ } \\
\text { Total }\end{array}$ & \multicolumn{1}{c}{ OR } & $\mathbf{9 5 \% ~ C l}$ & $\begin{array}{r}\text { Cases/ } \\
\text { Total }\end{array}$ & OR & $\mathbf{9 5 \% ~ C l}$ \\
\hline Derived fat mass $\mathbf{( k g )}$ & & & & & & \\
per 1 SD increase & $195 / 390$ & 0.89 & $(0.73,1.10)$ & $195 / 390$ & 1.03 & $(0.84,1.26)$ \\
Quartile 1 & $50 / 98$ & 1.00 & -- & $45 / 98$ & 1.00 & - \\
Quartile 2 & $53 / 97$ & 1.14 & $(0.65,2.01)$ & $44 / 97$ & 0.97 & $(0.55,1.71)$ \\
Quartile 3 & $44 / 97$ & 0.77 & $(0.43,1.36)$ & $55 / 97$ & 1.53 & $(0.87,2.72)$ \\
Quartile 4 & $48 / 98$ & 0.88 & $(0.49,1.58)$ & $51 / 98$ & 1.27 & $(0.72,2.26)$ \\
\hline
\end{tabular}

Estimates from unconditional logistic regression models adjusted for age (years, continuous), diabetes (yes/no), physical activity (METs/week, continuous), smoking status (ever/never), family history of prostate cancer (yes/no), and recent PSA screening (yes/no). Adiposity measures and metabolic scores were fit in separate models.

Quartiles of BMI: [18.6, 23.2], (23.2, 25.2], (25.2, 27.5], (27.5, 42.4].

Quartiles of BMI metabolic score: [20.5, 24.4], (24.4, 25.4], (25.4, 26.6], (26.6, 30.1].

Quartiles of waist circumference: [73.7, 88.9], (88.9, 94.6], (94.6, 102.0], (102.0, 135.0].

Quartiles of waist circumference metabolic score: [84.6, 92.4], (92.4, 95.1], (95.1, 97.7], (97.7, 108.0].

Quartiles of derived fat mass: [7.9, 17.8], (17.8, 21.4], $(21.4,25.3],(25.3,45.9]$.

Quartiles of fat mass metabolic score: [10.8,19.3], (19.3,21.5], $(21.5,24.0],(24.0,33.9]$.

Standard deviation of derived fat mass: $6.2 \mathrm{~kg}$.

Standard deviation of fat mass metabolic score: $3.7 \mathrm{~kg}$.

Standard deviation of waist circumference: $10.2 \mathrm{~cm}$.

Standard deviation of waist circumference metabolic score: $4.0 \mathrm{~cm}$. 
Table 3.4 Stratified analysis by time to diagnosis: Estimated odds ratios (OR) and 95\% confidence intervals (Cls) of advanced prostate cancer by self-reported adiposity and metabolic scores (predicted adiposity using metabolites), Health Professionals Follow-up Study.

\begin{tabular}{|c|c|c|c|c|c|c|c|c|}
\hline & \multicolumn{4}{|c|}{$<5.5$ years to diagnosis } & \multicolumn{4}{|c|}{$\geq 5.5$ years to diagnosis } \\
\hline & \multicolumn{2}{|c|}{ Adiposity measure } & \multicolumn{2}{|c|}{ Metabolic score } & \multicolumn{2}{|c|}{ Adiposity measure } & \multicolumn{2}{|c|}{ Metabolic score } \\
\hline & OR & $95 \% \mathrm{Cl}$ & OR & $95 \% \mathrm{Cl}$ & OR & $95 \% \mathrm{Cl}$ & OR & $95 \% \mathrm{Cl}$ \\
\hline \multicolumn{9}{|c|}{ Body mass index $\left(\mathrm{kg} / \mathrm{m}^{2}\right)$} \\
\hline per $5 \mathrm{~kg} / \mathrm{m}^{2}$ increase & 1.21 & $(0.80,1.83)$ & 1.48 & $(0.60,3.72)$ & 0.85 & $(0.55,1.31)$ & 1.27 & $(0.52,3.13)$ \\
\hline p-heterogeneity & & & & & & 0.13 & & 0.89 \\
\hline Quartile 1 & 1.00 & -- & 1.00 & -- & 1.00 & -- & 1.00 & -- \\
\hline Quartile 2 & 1.75 & $(0.75,4.15)$ & 0.92 & $(0.40,2.13)$ & 2.10 & $(0.97,4.60)$ & 0.62 & $(0.28,1.34)$ \\
\hline Quartile 3 & 1.99 & $(0.87,4.64)$ & 1.19 & $(0.51,2.77)$ & 0.83 & $(0.38,1.82)$ & 0.71 & $(0.32,1.55)$ \\
\hline Quartile 4 & 1.99 & $(0.88,4.58)$ & 1.59 & $(0.67,3.84)$ & 0.60 & $(0.26,1.37)$ & 1.29 & $(0.60,2.83)$ \\
\hline p-heterogeneity & & & & & & 0.05 & & 0.83 \\
\hline \multicolumn{9}{|c|}{ Waist circumference (cm) } \\
\hline per $1 S D$ increase & 0.92 & $(0.69,1.23)$ & 1.04 & $(0.77,1.40)$ & 0.88 & $(0.65,1.19)$ & 0.94 & $(0.70,1.26)$ \\
\hline p-heterogeneity & & & & & & 0.89 & & 0.67 \\
\hline Quartile 1 & 1.00 & -- & 1.00 & -- & 1.00 & -- & 1.00 & -- \\
\hline Quartile 2 & 1.06 & $(0.45,2.49)$ & 1.22 & $(0.52,2.87)$ & 1.51 & $(0.68,3.44)$ & 1.08 & $(0.51,2.31)$ \\
\hline Quartile 3 & 1.29 & $(0.54,3.12)$ & 0.85 & $(0.38,1.91)$ & 0.71 & $(0.34,1.46)$ & 1.27 & $(0.56,2.87)$ \\
\hline Quartile 4 & 0.81 & $(0.34,1.96)$ & 1.55 & $(0.68,3.61)$ & 0.97 & $(0.42,2.23)$ & 0.89 & $(0.40,1.96)$ \\
\hline p-heterogeneity & & & & & & 0.42 & & 0.53 \\
\hline
\end{tabular}


Table 3.4 (Continued) Stratified analysis by time to diagnosis: Estimated odds ratios (OR) and 95\% confidence intervals (Cls) of advanced prostate cancer by self-reported adiposity and metabolic scores (predicted adiposity using metabolites), Health Professionals Follow-up Study.

\begin{tabular}{|c|c|c|c|c|c|c|c|c|}
\hline & \multicolumn{4}{|c|}{$<5.5$ years to diagnosis } & \multicolumn{4}{|c|}{$\geq 5.5$ years to diagnosis } \\
\hline & \multicolumn{2}{|c|}{ Adiposity measure } & \multicolumn{2}{|c|}{ Metabolic score } & \multicolumn{2}{|c|}{ Adiposity measure } & \multicolumn{2}{|c|}{ Metabolic score } \\
\hline & OR & $95 \% \mathrm{Cl}$ & OR & $95 \% \mathrm{Cl}$ & OR & $95 \% \mathrm{Cl}$ & OR & $95 \% \mathrm{Cl}$ \\
\hline \multicolumn{9}{|c|}{ Derived fat mass $(\mathrm{kg})$} \\
\hline per $1 \mathrm{SD}$ increase & 0.95 & $(0.71,1.28)$ & 1.04 & $(0.78,1.38)$ & 0.78 & $(0.56,1.07)$ & 1.01 & $(0.74,1.38)$ \\
\hline p-heterogeneity & & & & & & 0.43 & & 0.95 \\
\hline Quartile 1 & 1.00 & -- & 1.00 & -- & 1.00 & -- & 1.00 & -- \\
\hline Quartile 2 & 1.30 & $(0.54,3.16)$ & 1.59 & $(0.68,3.77)$ & 0.93 & $(0.41,2.13)$ & 0.52 & $(0.22,1.21)$ \\
\hline Quartile 3 & 1.04 & $(0.42,2.54)$ & 1.75 & $(0.73,4.26)$ & 0.53 & $(0.24,1.19)$ & 1.21 & $(0.54,2.71)$ \\
\hline Quartile 4 & 1.04 & $(0.43,2.50)$ & 1.65 & $(0.69,3.99)$ & 0.65 & $(0.27,1.54)$ & 0.94 & $(0.41,2.16)$ \\
\hline p-heterogeneity & & & & & & 0.79 & & 0.30 \\
\hline
\end{tabular}

Estimates from unconditional logistic regression models adjusted for age (years, continuous), diabetes (yes/no), physical activity (METs/week, continuous), smoking status (ever/never), family history of prostate cancer (yes/no), and recent PSA screening (yes/no).

$<5.5$ years to diagnosis: 195 men (99 cases) contributed to BMI models, 199 men (100 cases) to waist circumference models, 189 men (98 cases) to fat mass models.

$\geq 5.5$ years to diagnosis: 203 men (102 cases) contributed to BMI models, 204 men (103 cases) to waist circumference models, 187 men (97 cases) to fat mass models. 
Figures 2.1-2.3 show estimated odds ratios for men cross-classified by selfreported adiposity and metabolic scores. For waist circumference and derived fat mass, the estimated odds of advanced prostate cancer were highest among men with a low self-reported adiposity but high metabolic score. However, confidence intervals were wide and overlapping. These findings were qualitatively similar under alternate classification decisions (Appendix 3.4).

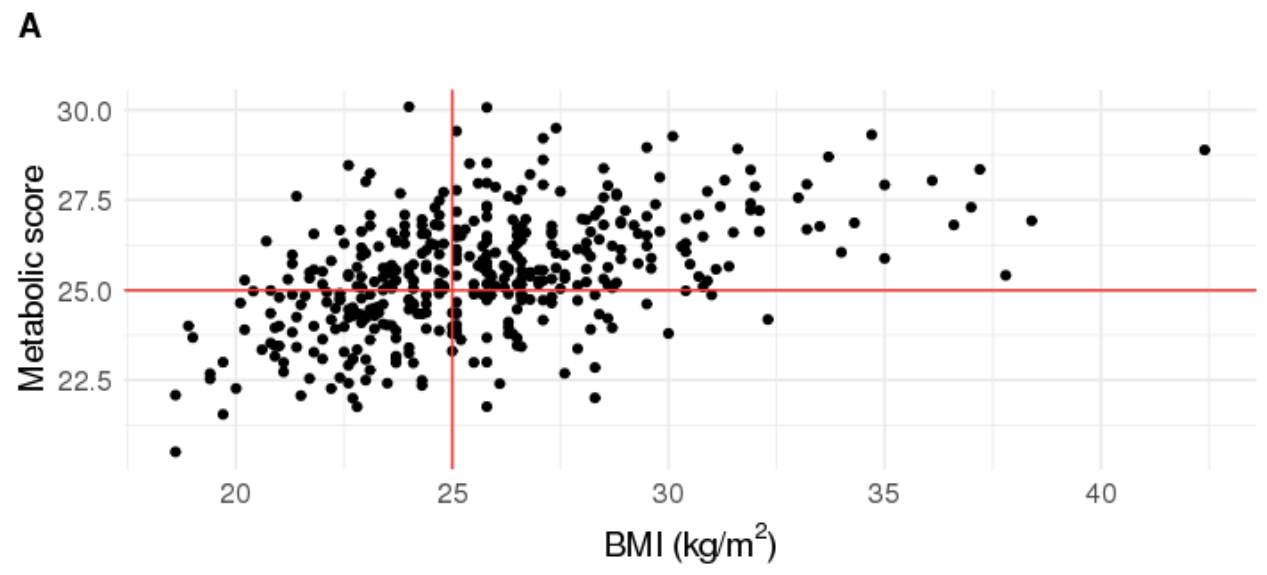

B Odds ratio for advanced prostate cancer by BMI-metabolic group

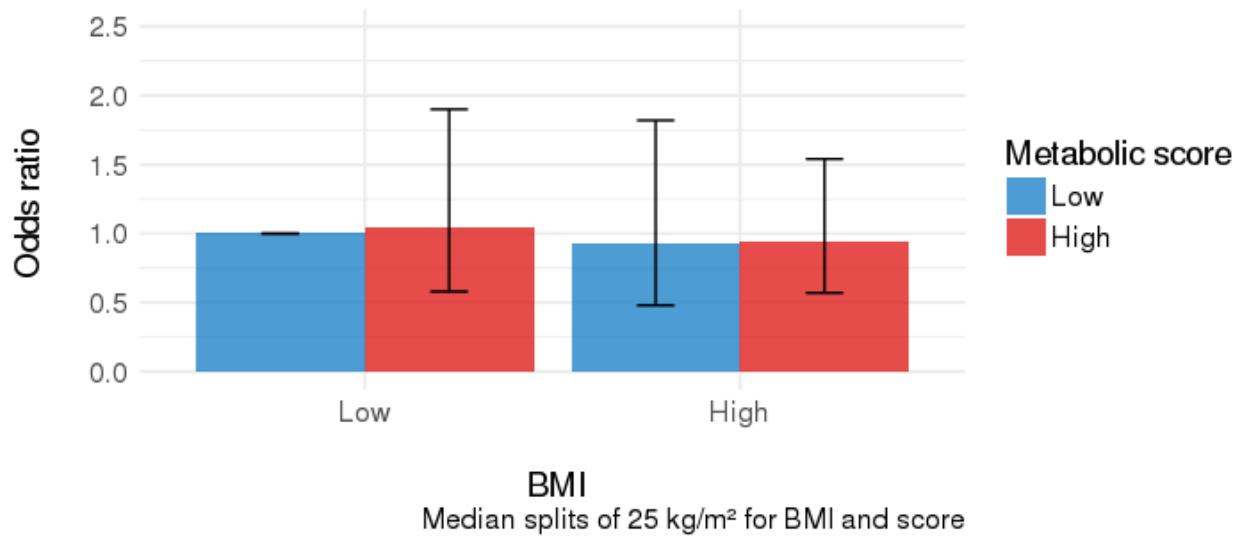

Figure 2.1 BMI-metabolic groups and advanced prostate cancer. Panel A: Scatterplot of self-reported BMI vs. metabolic score (predicted BMI based on metabolites) with median split lines drawn in red. Men were classified into BMI-metabolic groups based on having a self-reported BMI above vs. below the median and a metabolic score above vs. below the median. Panel B: Odds ratios for advanced prostate cancer by BMImetabolic group. Estimates adjusted for age, diabetes, physical activity, smoking status, family history of prostate cancer, and recent PSA screening. 


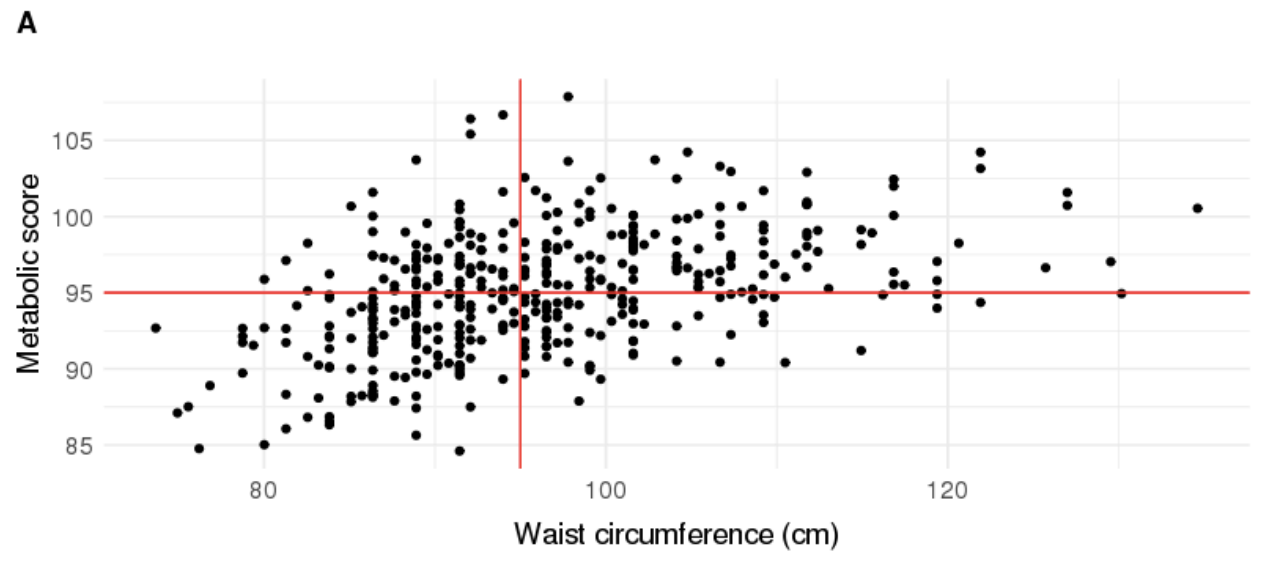

B Odds ratio for advanced prostate cancer by waist circumference-metabolic group

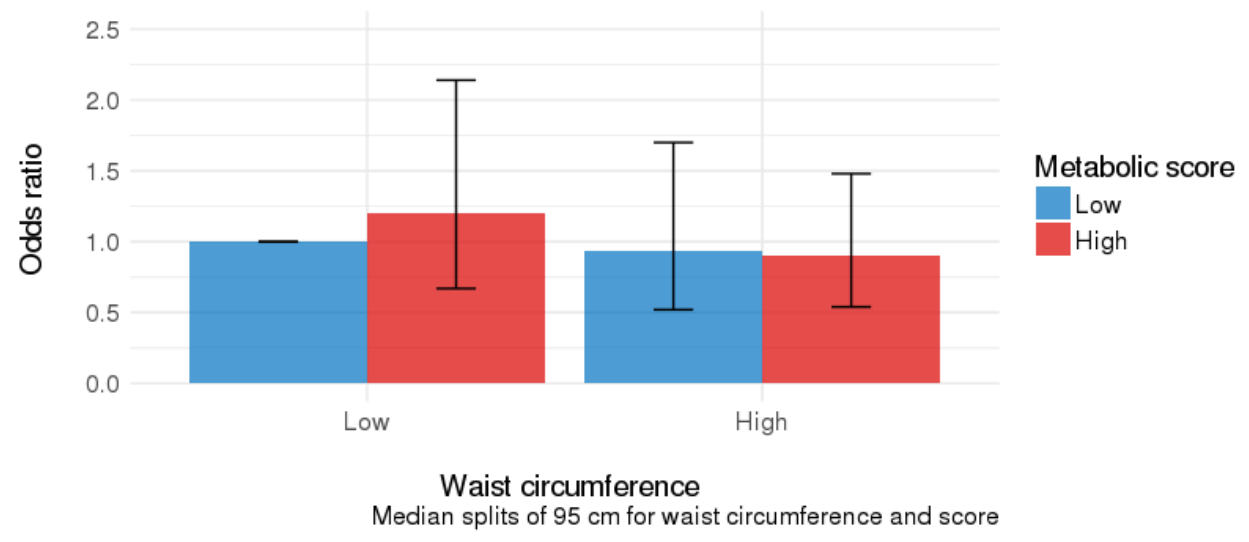

Figure 2.2 Waist circumference-metabolic groups and advanced prostate cancer. Panel A: Scatterplot of self-reported waist circumference vs. metabolic score (predicted waist circumference based on metabolites) with median split lines drawn in red. Men were classified into waist circumference-metabolic groups based on having a self-reported waist circumference above vs. below the median and a metabolic score above vs. below the median. Panel B: Odds ratios for advanced prostate cancer by waist circumference-metabolic group. Estimates adjusted for age, diabetes, physical activity, smoking status, family history of prostate cancer, and recent PSA screening. 


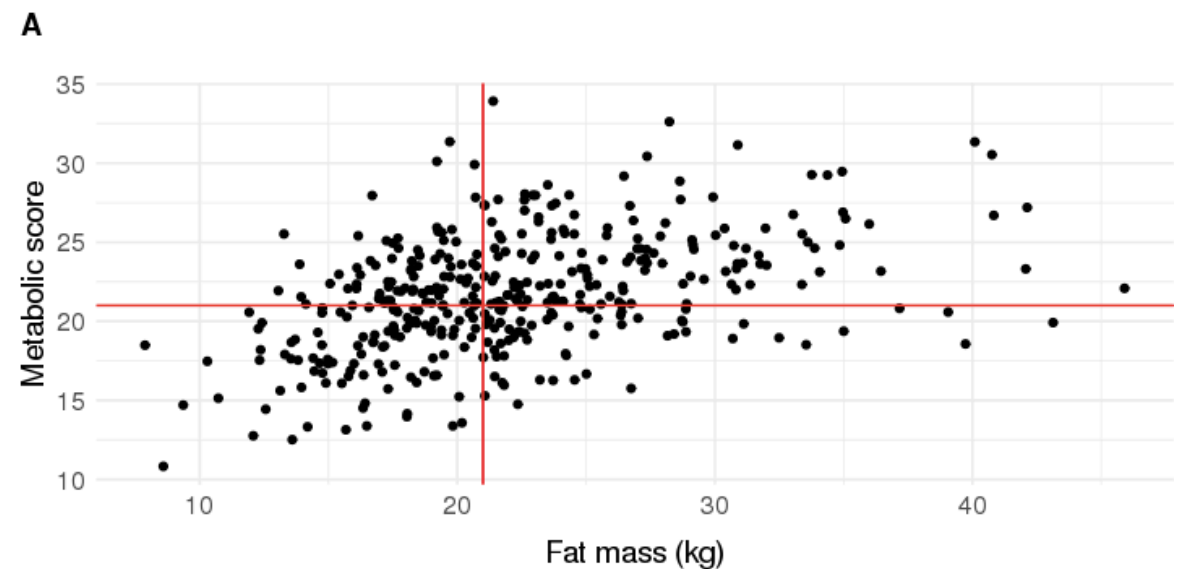

B Odds ratio for advanced prostate cancer by fat mass-metabolic group

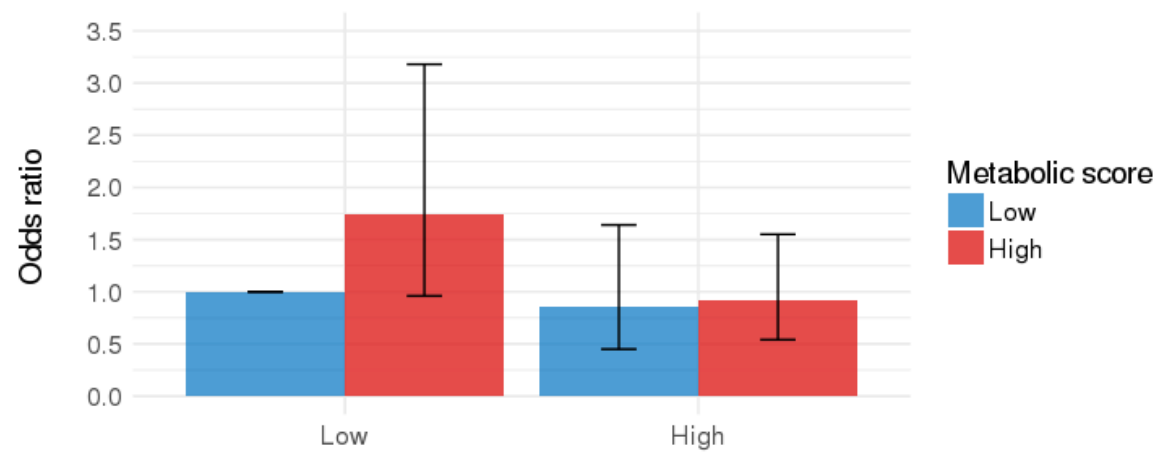

Fat mass

Median splits of $21 \mathrm{~kg}$ for fat mass and score

Figure 2.3 Fat mass-metabolic groups and advanced prostate cancer. Panel A: Scatterplot of derived fat mass vs. metabolic score (predicted fat mass based on metabolites) with median split lines drawn in red. Men were classified into fat massmetabolic groups based on having a derived fat mass above vs. below the median and a metabolic score above vs. below the median. Panel B: Odds ratios for advanced prostate cancer by fat mass-metabolic group. Estimates adjusted for age, diabetes, physical activity, smoking status, family history of prostate cancer, and recent PSA screening. 


\section{DISCUSSION}

In this metabolomics analysis of 198 identified metabolites, we found that 50 metabolites were associated with BMI, 43 with waist circumference, and 35 with derived fat mass, with considerable overlap across adiposity measures. In an independent set of men, neither adiposity nor metabolic scores representing metabolic obesity were associated with risk of advanced prostate cancer. However, estimates for self-reported $\mathrm{BMI}$ and risk of advanced prostate cancer were higher among men within 5.5 years of diagnosis. Further, we found suggestive evidence that men with a low waist circumference or fat mass but high metabolic score were at the highest risk of advanced disease, although power for these analyses was lower.

Among lipid metabolites, we identified inverse associations for CE, LPC, and LPE, positive associations for DAG, TAG, and SM, and mixed associations for PC with adiposity measures. These findings are largely consistent with a cross-sectional study of 217 metabolites among 2,383 Framingham Offspring participants, with the exception of CE and TAG, which showed positive and mixed associations in that study, respectively. ${ }^{4}$ That study also found that LPC $18: 2$ was the lipid metabolite most strongly associated with $\mathrm{BMI}$ and waist circumference,${ }^{4}$ in line with our findings for BMI and derived fat mass. A prospective study identified low LPC 18:2 as a predictor of incident pre-diabetes and diabetes over a 7-year period, independent of BMI. ${ }^{16}$

We found that carnitines were positively associated with all adiposity measures. Carnitine, which can be endogenously synthesized or absorbed from dietary sources such as meat, plays an important role in energy production by transporting long-chain fatty acids into the mitochondria for $\beta$-oxidation. ${ }^{17,18}$ Acylcarnitine accumulation may 
result from fatty acid oxidation defects in obese and insulin resistant individuals. ${ }^{19} \mathrm{We}$ found that waist circumference and derived fat mass were associated with two acylcarnitines not associated with BMI: $\mathrm{C} 16$ and $\mathrm{C} 12: 1$. A recent cross-sectional study reported that higher levels of these two metabolites may help distinguish overweight individuals with high versus low visceral fat area ( $\geq 100 \mathrm{vs.}<100 \mathrm{~cm}^{2}$ at L4). ${ }^{20}$

Among amino acids, we identified positive associations for glutamate and inverse associations for glutamine and glycine with all adiposity measures, which is consistent with previous findings. ${ }^{4,6-10}$ Several studies have also identified positive associations between branched chain amino acids (valine, leucine, isoleucine) and aromatic amino acids (tyrosine, phenylalaine) with BMI and/or waist circumference..$^{4,6,7,9}$ All but isoleucine were included in our study, but we did not replicate those findings. This may be due to a different distribution of BMI or modifiers, such as diet and physical activity, in our study population compared with others.

Prospective studies have reported associations between glutamate, glutamine, and glycine with future diabetes risk, ${ }^{6,21}$ and experimental studies have demonstrated that glutamine supplementation in humans and mice leads to improved glucose tolerance. ${ }^{6,22}$ This suggests that some of these metabolites may not only be biomarkers of obesity but also effectors of its later sequelae. While we found no evidence for an association between metabolically-defined obesity and advanced prostate cancer in the present study, the identified metabolites may prove relevant for other disease outcomes.

Prior meta-analyses reported an advanced prostate cancer relative risk of 1.09 (95\% Cl: 1.02-1.16; 13 studies) and a prostate cancer-specific mortality relative risk of 
1.15 (95\% Cl: $1.06-1.25 ; 6$ studies) per $5 \mathrm{~kg} / \mathrm{m}^{2}$ increase in BMI. ${ }^{1,23}$ These estimates are higher than our findings for advanced/fatal disease (OR 0.96, 95\% Cl: 0.73-1.27), but our power was limited by a small sample. Different estimates across studies may also be related to the timing of BMI measurement, length of follow-up, and patient characteristics.

We found that estimates for self-reported BMI and odds of advanced prostate cancer were higher among men within 5.5 years of diagnosis. This may represent an etiologically relevant time window during which obesity promotes the development of advanced disease. Alternatively, this may be partially due to detection bias, whereby obese men may experience delayed detection (due to lower PSA values and biopsy accuracy) and therefore more advanced disease at diagnosis than leaner men..$^{24,25}$ This may be more pronounced among men diagnosed earlier versus later in the study period, because obesity measured at baseline is more likely to reflect obesity at diagnosis for these men.

Those with the same measured obesity may differ in their underlying metabolic health,,$^{2-4}$ which could be relevant for later disease risk. We took a novel approach to address this by calculating metabolic scores to summarize underlying metabolic obesity. After cross-classifying men by self-reported and metabolically-defined obesity, we found that men at the highest risk of advanced prostate cancer were those who had a low selfreported adiposity but high metabolic score. The determinants of a normal weight, metabolically abnormal profile are unknown, but may be related to genetic factors influencing adipocyte function, body fat distribution, and insulin resistance and/or lifestyle factors such as physical activity and diet. ${ }^{5,26}$ Men in this group may have a 
strong propensity for dysregulated metabolism, given their unfavorable metabolic factors despite normal weight, which may contribute to the development and progression of advanced prostate cancer. ${ }^{27}$ Further investigation of this phenotype may provide additional insight into the underlying mechanisms and potential intervention targets for clinically important prostate cancer.

The major strength of our study was the integration of metabolomics with detailed clinical and lifestyle data within a well-characterized prospective cohort. This allowed us to investigate several adiposity measures and many metabolites while adjusting for important covariates. It also allowed us to assess adiposity and metabolites before advanced prostate cancer diagnosis to establish a temporal relationship and eliminate the potential for recall bias.

We relied on self-reported adiposity measures, which are subject to measurement error. However, a previous validation study in HPFS showed that selfreported weights and waist circumferences were highly correlated with technicianmeasured values (Pearson $r=0.97$ and $r=0.95$, respectively). ${ }^{11}$ We also relied on a single measurement of adiposity and metabolites in midlife, and it is possible that longterm average measures are the most biologically relevant for prostate cancer risk. Nonetheless, a pilot study showed that approximately $90 \%$ of metabolites were reproducible over two years within individuals ${ }^{14}$ and we estimated Pearson correlation coefficients $>0.90$ for BMI reports up to six years apart among men in the prostate cancer nested case-control study (data not shown). This suggests that a single measure may be reasonably representative of average values over midlife. Any error in assessing these average values is expected to be independent from the rate of prostate 
cancer and therefore attenuate our estimates. Our power for analyses of advanced prostate cancer was limited by the sample size. Lastly, our study population consisted of middle-aged or older health professionals of predominantly European ancestry, so our estimates may not be generalizable to other populations with different distributions of adiposity or risk factors.

In summary, BMI, waist circumference, and derived fat mass were associated with a broad range of metabolic alterations, involving lipids, amino acids, and amino acid derivatives. While neither adiposity nor metabolic scores were associated with advanced prostate cancer, the identified metabolites may inform future research on the mechanisms linking obesity with various disease outcomes. 


\section{Chapter 1 References}

1. Cao Y, Ma J. Body mass index, prostate cancer-specific mortality, and biochemical recurrence: a systematic review and meta-analysis. Cancer Prev Res (Phila). 2011;4(4):486-501.

2. Pischon $\mathrm{T}$, Boeing $\mathrm{H}$, Weikert $\mathrm{S}$, et al. Body size and risk of prostate cancer in the European Prospective Investigation into Cancer and Nutrition. Cancer Epidemiol Biomarkers Prev. 2008;17(11):3252-3261.

3. WCRF/AICR. Continuous Update Project Report: Diet, Nutrition, Physical Activity, and Prostate Cancer. 2014; http://www.wcrf.org/sites/default/files/Prostate-Cancer-2014-Report.pdf.

4. von Hafe $P$, Pina F, Perez A, Tavares $M$, Barros $H$. Visceral fat accumulation as a risk factor for prostate cancer. Obes Res. 2004;12(12):1930-1935.

5. Allott EH, Howard LE, Song HJ, et al. Racial differences in adipose tissue distribution and risk of aggressive prostate cancer among men undergoing radiotherapy. Cancer Epidemiol Biomarkers Prev. 2014;23(11):2404-2412.

6. Ibrahim MM. Subcutaneous and visceral adipose tissue: structural and functional differences. Obes Rev. 2010;11(1):11-18.

7. Nielsen TL, Hagen C, Wraae K, et al. Visceral and subcutaneous adipose tissue assessed by magnetic resonance imaging in relation to circulating androgens, sex hormone-binding globulin, and luteinizing hormone in young men. $J$ Clin Endocrinol Metab. 2007;92(7):2696-2705.

8. Kim C, Dabelea D, Kalyani RR, et al. Changes in visceral adiposity, subcutaneous adiposity, and sex hormones in the Diabetes Prevention Program. J Clin Endocrinol Metab. 2017.

9. Goodpaster $\mathrm{BH}$, Krishnaswami S, Resnick H, et al. Association between regional adipose tissue distribution and both type 2 diabetes and impaired glucose tolerance in elderly men and women. Diabetes Care. 2003;26(2):372-379.

10. Snijder MB, Dekker JM, Visser M, et al. Trunk fat and leg fat have independent and opposite associations with fasting and postload glucose levels: the Hoorn Study. Diabetes Care. 2004;27(2):372-377.

11. Karpe F, Pinnick KE. Biology of upper-body and lower-body adipose tissue--link to whole-body phenotypes. Nat Rev Endocrinol. 2015;11(2):90-100. 
12. Giovannucci E, Michaud D. The role of obesity and related metabolic disturbances in cancers of the colon, prostate, and pancreas. Gastroenterology. 2007;132(6):2208-2225.

13. Platz EA, Leitzmann MF, Rifai N, et al. Sex steroid hormones and the androgen receptor gene CAG repeat and subsequent risk of prostate cancer in the prostate-specific antigen era. Cancer Epidemiol Biomarkers Prev. 2005;14(5):1262-1269.

14. Harris TB, Launer LJ, Eiriksdottir G, et al. Age, Gene/Environment SusceptibilityReykjavik Study: multidisciplinary applied phenomics. Am J Epidemiol. 2007;165(9):1076-1087.

15. Wajchenberg BL. Subcutaneous and visceral adipose tissue: their relation to the metabolic syndrome. Endocr Rev. 2000;21(6):697-738.

16. Johannesdottir F, Aspelund T, Siggeirsdottir K, et al. Mid-thigh cortical bone structural parameters, muscle mass and strength, and association with lower limb fractures in older men and women (AGES-Reykjavik Study). Calcif Tissue Int. 2012;90(5):354-364.

17. Sigurdardottir LG, Jonasson JG, Stefansdottir S, et al. Data quality at the Icelandic Cancer Registry: comparability, validity, timeliness and completeness. Acta Oncol. 2012;51(7):880-889.

18. Zilli T, Chagnon M, Van Nguyen T, et al. Influence of abdominal adiposity, waist circumference, and body mass index on clinical and pathologic findings in patients treated with radiotherapy for localized prostate cancer. Cancer. 2010;116(24):5650-5658.

19. Giovannucci E, Rimm EB, Liu Y, et al. Body mass index and risk of prostate cancer in U.S. health professionals. J Natl Cancer Inst. 2003;95(16):1240-1244.

20. Moller E, Wilson KM, Batista JL, Mucci LA, Balter K, Giovannucci E. Body size across the life course and prostate cancer in the Health Professionals Follow-up Study. Int J Cancer. 2016;138(4):853-865.

21. Hernandez BY, Park SY, Wilkens LR, Henderson BE, Kolonel LN. Relationship of body mass, height, and weight gain to prostate cancer risk in the Multiethnic Cohort. Cancer Epidemiol Biomarkers Prev. 2009;18(9):2413-2421.

22. Shuster A, Patlas M, Pinthus JH, Mourtzakis M. The clinical importance of visceral adiposity: a critical review of methods for visceral adipose tissue analysis. Br J Radiol. 2012;85(1009):1-10.

23. Wallstrom P, Bjartell A, Gullberg B, Olsson H, Wirfalt E. A prospective Swedish study on body size, body composition, diabetes, and prostate cancer risk. $\mathrm{Br} \mathrm{J}$ Cancer. 2009;100(11):1799-1805. 
24. Guerrios-Rivera L, Howard L, Frank J, et al. Is body mass index the best adiposity measure for prostate cancer risk? Results from a Veterans Affairs biopsy cohort. Urology. 2017;105:129-135.

25. Fowke JH, Motley SS, Concepcion RS, Penson DF, Barocas DA. Obesity, body composition, and prostate cancer. BMC Cancer. 2012;12:23.

26. De Marzo AM, Platz EA, Sutcliffe S, et al. Inflammation in prostate carcinogenesis. Nat Rev Cancer. 2007;7(4):256-269.

27. Li H, Stampfer MJ, Mucci L, et al. A 25-year prospective study of plasma adiponectin and leptin concentrations and prostate cancer risk and survival. Clin Chem. 2010;56(1):34-43.

28. Hrafnkelsdottir SM, Torfadottir JE, Aspelund T, et al. Physical activity from early adulthood and risk of prostate cancer: a 24-Year follow-up study among Icelandic men. Cancer Prev Res (Phila). 2015;8(10):905-911.

29. Rodriguez C, Freedland SJ, Deka A, et al. Body mass index, weight change, and risk of prostate cancer in the Cancer Prevention Study II Nutrition Cohort. Cancer Epidemiol Biomarkers Prev. 2007;16(1):63-69.

30. Putnam SD, Cerhan JR, Parker AS, et al. Lifestyle and anthropometric risk factors for prostate cancer in a cohort of lowa men. Ann Epidemiol. 2000;10(6):361-369.

31. Beebe-Dimmer JL, Faerber GJ, Morgenstern H, et al. Body composition and serum prostate-specific antigen: review and findings from Flint Men's Health Study. Urology. 2008;71(4):554-560.

32. Buschemeyer WC, $3^{\text {rd }}$, Freedland SJ. Obesity and prostate cancer: epidemiology and clinical implications. Eur Urol. 2007;52(2):331-343.

33. Seidell JC, Visscher TL. Body weight and weight change and their health implications for the elderly. Eur J Clin Nutr. 2000;54 Suppl 3:S33-39. 


\section{Chapter 2 References}

1. Kenfield SA, Stampfer MJ, Giovannucci E, Chan JM. Physical activity and survival after prostate cancer diagnosis in the Health Professionals Follow-up Study. J Clin Oncol. 2011;29(6):726-732.

2. Richman EL, Kenfield SA, Stampfer MJ, Paciorek A, Carroll PR, Chan JM. Physical activity after diagnosis and risk of prostate cancer progression: data from the Cancer of the Prostate Strategic Urologic Research Endeavor. Cancer Res. 2011;71(11):3889-3895.

3. Bonn SE, Sjolander A, Lagerros YT, et al. Physical activity and survival among men diagnosed with prostate cancer. Cancer Epidemiol Biomarkers Prev. 2015;24(1):57-64.

4. Wang Y, Jacobs EJ, Gapstur SM, et al. Recreational physical activity in relation to prostate cancer-specific mortality among men with nonmetastatic prostate cancer. Eur Urol. 2017.

5. Friedenreich CM, Wang Q, Neilson HK, Kopciuk KA, McGregor SE, Courneya KS. Physical activity and survival after prostate cancer. Eur Urol. 2016;70(4):576585.

6. Kenfield SA, Batista JL, Jahn JL, et al. Development and application of a lifestyle score for prevention of lethal prostate cancer. J Natl Cancer Inst. 2016;108(3).

7. Schmitz KH, Courneya KS, Matthews $\mathrm{C}$, et al. American College of Sports Medicine roundtable on exercise guidelines for cancer survivors. Med Sci Sports Exerc. 2010;42(7):1409-1426.

8. Pernar $\mathrm{CH}$, Fall $\mathrm{K}$, Rider JR, et al. A walking intervention among men with prostate cancer: a pilot study. Clin Genitourin Cancer. 2017.

9. Thorsen L, Courneya KS, Stevinson C, Fossa SD. A systematic review of physical activity in prostate cancer survivors: outcomes, prevalence, and determinants. Support Care Cancer. 2008;16(9):987-997.

10. Ligibel JA, Alfano CM, Hershman D, et al. Recommendations for obesity clinical trials in cancer survivors: American Society of Clinical Oncology statement. J Clin Oncol. 2015;33(33):3961-3967.

11. Visvanathan $\mathrm{K}$, Levit LA, Raghavan $\mathrm{D}$, et al. Untapped potential of observational research to inform clinical decision making: American Society of Clinical Oncology Research Statement. J Clin Oncol. 2017;35(16):1845-1854.

12. Hernán MA, Robins JM. Using big data to emulate a target trial when a randomized trial is not available. Am J Epidemiol. 2016;183(8):758-764. 
13. Chasan-Taber S, Rimm EB, Stampfer MJ, et al. Reproducibility and validity of a self-administered physical activity questionnaire for male health professionals. Epidemiology. 1996;7(1):81-86.

14. Rimm EB, Stampfer MJ, Colditz GA, Chute CG, Litin LB, Willett WC. Validity of self-reported waist and hip circumferences in men and women. Epidemiology. 1990;1(6):466-473.

15. Young JG, Hernán MA, Robins JM. Identification, estimation and approximation of risk under interventions that depend on the natural value of treatment using observational data. Epidemiol Methods. 2014;3(1):1-19.

16. Skolarus TA, Wolf AM, Erb NL, et al. American Cancer Society prostate cancer survivorship care guidelines. CA Cancer J Clin. 2014;64(4):225-249.

17. Hernán MA. Does water kill? A call for less casual causal inferences. Ann Epidemiol. 2016;26(10):674-680.

18. Hernán MA, VanderWeele TJ. Compound treatments and transportability of causal inference. Epidemiology. 2011;22(3):368-377.

19. Robins J, Hernán, MA. Estimation of the causal effects of time-varying exposures. In: Fitzmaurice G, Davidian, M, Verbeke, G, et al, ed. Longitudinal data analysis. Boca Raton, FL: Chapman \& Hall/CRC; 2009:553-599.

20. Hernán MA, Robins JM. Per-protocol analyses of pragmatic trials. N Engl J Med. 2017;377(14):1391-1398.

21. Taubman SL, Robins JM, Mittleman MA, Hernán MA. Intervening on risk factors for coronary heart disease: An application of the parametric g-formula. Int $\mathrm{J}$ Epidemiol. 2009;38(6):1599-1611.

22. Danaei G, Robins JM, Young JG, Hu FB, Manson JE, Hernán MA. Weight loss and coronary heart disease: sensitivity analysis for unmeasured confounding by undiagnosed disease. Epidemiology. 2016;27(2):302-310.

23. Lajous $\mathrm{M}$, Willett WC, Robins $\mathrm{J}$, et al. Changes in fish consumption in midlife and the risk of coronary heart disease in men and women. Am J Epidemiol. 2013;178(3):382-391.

24. Danaei G, Pan A, Hu FB, Hernán MA. Hypothetical midlife interventions in women and risk of type 2 diabetes. Epidemiology. 2013;24(1):122-128.

25. Vangen-Lonne AM, Ueda P, Gulayin P, Wilsgaard T, Mathiesen EB, Danaei G. Hypothetical interventions to prevent stroke: an application of the parametric gformula to a healthy middle-aged population. Eur J Epidemiol. 2018. 
26. Garcia-Aymerich J, Varraso R, Danaei G, Camargo CA, Jr., Hernán MA. Incidence of adult-onset asthma after hypothetical interventions on body mass index and physical activity: an application of the parametric g-formula. Am J Epidemiol. 2014;179(1):20-26.

27. Lipsitch M, Tchetgen Tchetgen E, Cohen T. Negative controls: a tool for detecting confounding and bias in observational studies. Epidemiology. 2010;21(3):383-388.

28. American Cancer Society. Cancer Facts \& Figures. https://www.cancer.org/content/dam/cancer-org/research/cancer-facts-andstatistics/annual-cancer-facts-and-figures/2017/cancer-facts-and-figures2017.pdf.

29. Epstein MM, Edgren G, Rider JR, Mucci LA, Adami HO. Temporal trends in cause of death among Swedish and US men with prostate cancer. J Natl Cancer Inst. 2012;104(17):1335-1342.

30. Newton RU, Kenfield SA, Hart NH, et al. Intense Exercise for Survival among Men with Metastatic Castrate-Resistant Prostate Cancer (INTERVAL-GAP4): a multicentre, randomised, controlled phase III study protocol. BMJ Open. 2018;8(5):e022899. 


\section{Chapter 3 References}

1. Cao Y, Ma J. Body mass index, prostate cancer-specific mortality, and biochemical recurrence: a systematic review and meta-analysis. Cancer Prev Res (Phila). 2011;4(4):486-501.

2. Stefan N, Schick F, Haring HU. Causes, characteristics, and consequences of metabolically unhealthy normal weight in humans. Cell Metab. 2017;26(2):292300.

3. Batch BC, Shah SH, Newgard CB, et al. Branched chain amino acids are novel biomarkers for discrimination of metabolic wellness. Metabolism. 2013;62(7):961969.

4. Ho JE, Larson MG, Ghorbani A, et al. Metabolomic profiles of body mass index in the Framingham Heart Study reveal distinct cardiometabolic phenotypes. PLoS One. 2016;11(2):e0148361.

5. Wildman RP, Muntner P, Reynolds K, et al. The obese without cardiometabolic risk factor clustering and the normal weight with cardiometabolic risk factor clustering: prevalence and correlates of 2 phenotypes among the US population (NHANES 1999-2004). Arch Intern Med. 2008;168(15):1617-1624.

6. Cheng S, Rhee EP, Larson MG, et al. Metabolite profiling identifies pathways associated with metabolic risk in humans. Circulation. 2012;125(18):2222-2231.

7. Moore SC, Matthews CE, Sampson JN, et al. Human metabolic correlates of body mass index. Metabolomics. 2014;10(2):259-269.

8. Moore SC, Playdon MC, Sampson JN, et al. A metabolomics analysis of body mass index and postmenopausal breast cancer risk. J Natl Cancer Inst. 2018;110(6):588-597.

9. Newgard CB, An J, Bain JR, et al. A branched-chain amino acid-related metabolic signature that differentiates obese and lean humans and contributes to insulin resistance. Cell Metab. 2009;9(4):311-326.

10. Ottosson F, Brunkwall L, Ericson U, et al. Connection between BMI-related plasma metabolite profile and gut microbiota. J Clin Endocrinol Metab. 2018;103(4):1491-1501.

11. Rimm EB, Stampfer MJ, Colditz GA, Chute CG, Litin LB, Willett WC. Validity of self-reported waist and hip circumferences in men and women. Epidemiology. 1990;1(6):466-473.

12. Lee DH, Keum N, Hu FB, et al. Development and validation of anthropometric prediction equations for lean body mass, fat mass and percent fat in adults using 
the National Health and Nutrition Examination Survey (NHANES) 1999-2006. $\mathrm{Br}$ J Nutr. 2017;118(10):858-866.

13. Paynter NP, Balasubramanian R, Giulianini F, et al. Metabolic predictors of incident coronary heart disease in women. Circulation. 2018;137(8):841-853.

14. Townsend MK, Clish CB, Kraft P, et al. Reproducibility of metabolomic profiles among men and women in 2 large cohort studies. Clin Chem. 2013;59(11):16571667.

15. Benjamini $Y$, Hochberg, Y. Controlling the false discovery rate: A practical and powerful approach to multiple testing. JR Statist Soc B. 1995;57:289-300.

16. Wang-Sattler R, Yu Z, Herder C, et al. Novel biomarkers for pre-diabetes identified by metabolomics. Mol Syst Biol. 2012;8:615.

17. Rebouche C. Carnitine. In: Shils ME SM, Ross AC, Caballero B, Cousins RJ, ed. Modern Nutrition in Health and Disease. 10th ed. Philadelphia: Lippincott, Williams \& Wilkins; 2006:537-544.

18. Rebouche CJ. Kinetics, pharmacokinetics, and regulation of L-carnitine and acetyl-L-carnitine metabolism. Ann N Y Acad Sci. 2004;1033:30-41.

19. Mihalik SJ, Goodpaster BH, Kelley DE, et al. Increased levels of plasma acylcarnitines in obesity and type 2 diabetes and identification of a marker of glucolipotoxicity. Obesity (Silver Spring). 2010;18(9):1695-1700.

20. Baek SH, Kim M, Kim M, et al. Metabolites distinguishing visceral fat obesity and atherogenic traits in individuals with overweight. Obesity (Silver Spring). 2017;25(2):323-331.

21. Floegel A, Stefan N, Yu Z, et al. Identification of serum metabolites associated with risk of type 2 diabetes using a targeted metabolomic approach. Diabetes. 2013;62(2):639-648.

22. Greenfield JR, Farooqi IS, Keogh JM, et al. Oral glutamine increases circulating glucagon-like peptide 1, glucagon, and insulin concentrations in lean, obese, and type 2 diabetic subjects. Am J Clin Nutr. 2009;89(1):106-113.

23. Discacciati A, Orsini N, Wolk A. Body mass index and incidence of localized and advanced prostate cancer--a dose-response meta-analysis of prospective studies. Ann Oncol. 2012;23(7):1665-1671.

24. Beebe-Dimmer JL, Faerber GJ, Morgenstern H, et al. Body composition and serum prostate-specific antigen: review and findings from Flint Men's Health Study. Urology. 2008;71(4):554-560. 
25. Buschemeyer WC, 3rd, Freedland SJ. Obesity and prostate cancer: epidemiology and clinical implications. Eur Urol. 2007;52(2):331-343.

26. Scott RA, Fall T, Pasko D, et al. Common genetic variants highlight the role of insulin resistance and body fat distribution in type 2 diabetes, independent of obesity. Diabetes. 2014;63(12):4378-4387.

27. Giovannucci E, Michaud D. The role of obesity and related metabolic disturbances in cancers of the colon, prostate, and pancreas. Gastroenterology. 2007; 132(6):2208-2225. 


\section{APPENDIX 1}

Appendix Table 1.1 Distribution of adiposity measures at baseline for men in the AGES-Reykjavik Study, 2002

Appendix Table 1.2 Association between fat depots and risk of prostate cancer among men in the AGES-Reykjavik Study, 2002-2015: Additional adjustment for BMI 
Appendix Table 1.1 Distribution of adiposity measures at baseline for men in the AGESReykjavik Study, 2002

\begin{tabular}{lrrrrr}
\hline & $\mathrm{N}$ & Mean & $\begin{array}{r}\text { Standard } \\
\text { Deviation }\end{array}$ & Median & $\begin{array}{r}\text { Interquartile } \\
\text { Range }\end{array}$ \\
\hline Abdominal fat area ${ }^{1}\left(\mathrm{~cm}^{2}\right)$ & & & & & \\
$\quad$ Visceral & 1,832 & 205.8 & 85.7 & 194.6 & 110.7 \\
$\quad$ Subcutaneous & 1,832 & 204.1 & 85.6 & 192.7 & 106.3 \\
$\quad$ Thigh fat area ${ }^{1}\left(\mathrm{~cm}^{2}\right)$ & & & & & \\
$\quad$ Intermuscular & 1,832 & 35.4 & 16.0 & 32.5 & 19.1 \\
$\quad$ Subcutaneous & 1,832 & 78.5 & 39.2 & 70.7 & 47.2 \\
Total fat mass $^{2}(\mathrm{~kg})$ & 1,425 & 18.6 & 6.8 & 17.9 & 8.9 \\
Percent body fat $^{2}$ & 1,425 & 21.9 & 5.3 & 21.9 & 7.0 \\
BMI $^{3}\left(\mathrm{~kg} / \mathrm{m}^{2}\right)$ & 1,832 & 27.0 & 3.7 & 26.6 & 4.8 \\
Waist circumference $^{3}(\mathrm{~cm})$ & 1,832 & 102.5 & 10.3 & 102.0 & 13.0 \\
\hline
\end{tabular}

${ }^{1}$ Measured by CT imaging.

${ }^{2}$ Measured by bioelectric impedance.

${ }^{3}$ Measured by trained technicians. 


\begin{tabular}{|c|c|c|c|c|}
\hline \multicolumn{5}{|c|}{$\begin{array}{l}\text { Appendix Table } 1.2 \text { Association between fat depots and risk of prostate cancer among men in the AGES-Reykjavik Study, 2002-2015: } \\
\text { Additional adjustment for BMl }\end{array}$} \\
\hline & $\begin{array}{l}\text { Total prostate cancer } \\
\text { (172 events/1,832 men) }\end{array}$ & $\begin{array}{r}\text { High-grade prostate cancer } \\
\text { (43 events } / 1,832 \text { men) }\end{array}$ & $\begin{array}{r}\text { Advanced prostate cancer } \\
\text { (41 events/1,832 men) }\end{array}$ & $\begin{array}{l}\text { Fatal prostate cancer } \\
\text { (31 events/1,832 men) }\end{array}$ \\
\hline & $\begin{array}{l}\text { Fully-adjusted } \\
\mathrm{HR}(95 \% \mathrm{Cl})^{1}\end{array}$ & $\begin{array}{r}\text { Fully-adjusted } \\
\text { HR }(95 \% \mathrm{Cl})^{1}\end{array}$ & $\begin{array}{l}\text { Fully-adjusted } \\
\text { HR }(95 \% \mathrm{Cl})^{1}\end{array}$ & $\begin{array}{l}\text { Fully-adjusted } \\
\operatorname{HR}(95 \% \mathrm{Cl})^{1}\end{array}$ \\
\hline Abdominal visceral & $1.03(0.83,1.28)$ & $0.93(0.60,1.45)$ & $1.14(0.77,1.70)$ & $0.99(0.61,1.60)$ \\
\hline Abdominal subcutaneous & $0.89(0.68,1.17)$ & $1.04(0.61,1.77)$ & $0.85(0.50,1.44)$ & $0.90(0.49,1.65)$ \\
\hline Thigh intermuscular & $0.87(0.71,1.06)$ & $0.86(0.57,1.29)$ & $0.76(0.51,1.14)$ & $1.08(0.71,1.66)$ \\
\hline Thigh subcutaneous & $1.02(0.85,1.24)$ & $1.21(0.85,1.73)$ & $1.08(0.76,1.53)$ & $1.22(0.81,1.84)$ \\
\hline
\end{tabular}

HR: Hazard ratio; Cl: Confidence interval; BMI: Body mass index.

Continuous adiposity measures were modeled per 1 standard deviation (SD) increase: abdominal visceral fat $\left(85.7 \mathrm{~cm}^{2}\right)$, abdominal subcutaneous fat $\left(85.6 \mathrm{~cm}^{2}\right)$, thigh intermuscular $\left(16.0 \mathrm{~cm}^{2}\right)$, thigh subcutaneous $\left(39.2 \mathrm{~cm}^{2}\right)$.

${ }^{1}$ Adjusted for the following variables, measured at study entry: age (continuous), height (continuous), family history of prostate cancer (yes, no), smoking status (never, former, current), education (primary/secondary, college/university), physical activity ( $\leq 3$ hours/week, $\geq 4$ hours/week), physician visit over past 12 months (yes, no), and BMI (continuous). 
Appendix 2.1

Appendix Table 2.1 Specification and emulation of the target trial 83

\section{Appendix 2.2}

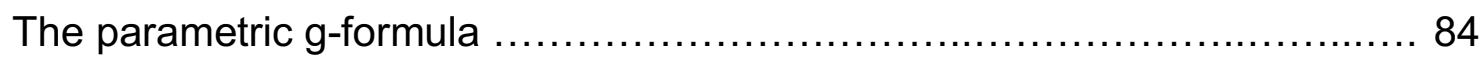

Appendix Table 2.2.1 Covariates ....................................... 86

Appendix Table 2.2.2 Cumulative and average percent of men

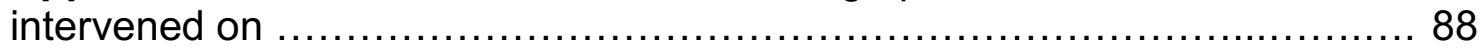

Appendix Figure 1 Mean difference between observed vs. simulated (under no intervention) values of time-varying covariates by year of follow-up

Appendix 2.3 - Results from additional analyses

Appendix Table 2.3.1 Estimated survival time and time invested in physical activity ....

Appendix Table 2.3.2 Estimated risk ratios with risk under low activity as the reference 93

Appendix Table 2.3.3 Pooled logistic regression

Appendix 2.4 - Results from sensitivity analyses

Appendix Table 2.4.1 Negative outcome control ............................. 95

Appendix Table 2.4.2 Assuming that the development of functional impairment, metastasis, and chronic diseases occurred 2 years before the questionnaire in which these conditions were reported 96

Appendix Table 2.4.3 Keeping the total time devoted to vigorous and moderate activity constant 97

Appendix Table 2.4.4 Imposing a two-year lag time 98

\section{Supplemental material}

Models used for the parametric g-formula 99 


\section{Appendix 2.1}

\begin{tabular}{|c|c|c|}
\hline \multicolumn{3}{|c|}{$\begin{array}{l}\text { Appendix Table } 2.1 \text { Specification and emulation of a target trial of physical activity interventions } \\
\text { among men with nonmetastatic prostate cancer using data from the Health Professionals Follow-up } \\
\text { Study }\end{array}$} \\
\hline Component & Target trial & $\begin{array}{l}\text { Emulation using } \\
\text { observational data }\end{array}$ \\
\hline Aim & $\begin{array}{l}\text { To estimate the effect of physical activity } \\
\text { interventions on } 10 \text {-year risk of mortality among } \\
\text { U.S. men aged } 50-80 \text { years and diagnosed with } \\
\text { nonmetastatic prostate cancer. }\end{array}$ & Same. \\
\hline $\begin{array}{l}\text { Eligibility } \\
\text { criteria }\end{array}$ & $\begin{array}{l}\text { Men aged } 50-80 \text { years diagnosed with } \\
\text { nonmetastatic prostate cancer between Jan } \\
1998 \text { and Jan } 2010 \text {. Exclusion criteria of recent } \\
\text { cardiovascular event (myocardial infarction or } \\
\text { stroke), congestive heart failure, amyotrophic } \\
\text { lateral sclerosis, or functional impairment } \\
\text { (difficulty climbing a flight of stairs or walking } \\
\text { eight blocks). }\end{array}$ & Same. \\
\hline $\begin{array}{l}\text { Treatment } \\
\text { strategies }\end{array}$ & $\begin{array}{l}\text { Initiate physical activity intervention at diagnosis } \\
\text { and continue it over follow-up until the } \\
\text { development of metastasis or a condition or } \\
\text { disease limiting physical ability, including } \\
\text { metastasis, myocardial infarction, stroke, } \\
\text { congestive heart failure, amyotrophic lateral } \\
\text { sclerosis, or functional impairment. }\end{array}$ & $\begin{array}{l}\text { Same, except baseline is } \\
\text { taken as the return date of the } \\
\text { first questionnaire after } \\
\text { prostate cancer diagnosis. }\end{array}$ \\
\hline $\begin{array}{l}\text { Treatment } \\
\text { assignment }\end{array}$ & $\begin{array}{l}\text { At diagnosis, men assigned to a strategy and } \\
\text { will be aware of the strategy to which they have } \\
\text { been assigned. }\end{array}$ & Same. \\
\hline Follow-up & $\begin{array}{l}\text { Starts at diagnosis and ends at death, loss to } \\
\text { follow-up, } 10 \text { years after baseline, or on } 1 \text { June } \\
2014 \text {, whichever occurs first. }\end{array}$ & $\begin{array}{l}\text { Same, except baseline is } \\
\text { taken as the return date of the } \\
\text { first questionnaire after } \\
\text { prostate cancer diagnosis. }\end{array}$ \\
\hline Outcome & All-cause mortality within 10 years of diagnosis. & Same. \\
\hline $\begin{array}{l}\text { Causal } \\
\text { contrast of } \\
\text { interest }\end{array}$ & $\begin{array}{l}\text { Intention-to-treat effect, i.e., effect of being } \\
\text { assigned to a physical activity strategy vs. no } \\
\text { strategy at baseline. Per-protocol effect, i.e., } \\
\text { effect that would have been observed if all men } \\
\text { adhered to their assigned strategy over the 10- } \\
\text { year follow-up. }\end{array}$ & $\begin{array}{l}\text { Observational analog of per- } \\
\text { protocol effect. }\end{array}$ \\
\hline $\begin{array}{l}\text { Statistical } \\
\text { analysis }\end{array}$ & $\begin{array}{l}\text { Intention-to-treat analysis. } \\
\text { Per-protocol analysis: comparison of } 10 \text {-year } \\
\text { risk of death between groups receiving each } \\
\text { treatment strategy with adjustment for pre- and } \\
\text { post-baseline prognostic factors associated with } \\
\text { adherence to the treatment strategies and loss } \\
\text { to follow-up. }\end{array}$ & Same as per-protocol analysis. \\
\hline
\end{tabular}




\section{Appendix 2.2}

The g-formula risk is a weighted average of risks conditional on a specified intervention history and observed confounder history. The weights are the probability density functions of the time-varying confounders, which are estimated using parametric regression models, and the weighted average is approximated using Monte Carlo simulation (of 10,000 men in our analyses). The adjustment covariates are described in Appendix Table 2.1.

To assess model misspecification, we compared the observed means of time-varying covariates with the model-based predicted means under no intervention (Appendix Figure 1). The estimated 10-year risk of death under no physical activity intervention was the same as the observed risk (15.4\%), a necessary condition for no model misspecification, and the estimated and observed means of all time-varying covariates were close.

To assess the possibility of model extrapolation, we calculated the proportion of men who had to increase their activity at any point during follow-up and during each two-year period on average in order to keep adhering to the strategy (Appendix Table 2.2.2). The cumulative proportion of men who would have to change their behavior at any point over follow-up to keep adhering to the strategies was below $100 \%$ for all interventions. When we expanded the set of chronic conditions that would excuse men from following the recommended physical activity levels, the proportion of men who had to change their behavior at any time over follow-up to remain adherent to these strategies decreased, as expected.

Additionally, we estimated risk ratios with risk under low activity $(<1.25$ hours/week of vigorous or $<2.5$ hours/week of moderate) as the reference (Appendix Table 2.3.2). For comparison with a conventional approach, we fit pooled logistic models to estimate the association between physical activity and risk of death in two-year intervals (Appendix Table

2.3.3). As expected, estimated risk ratios were further from the null when risk under low physical activity was used as the reference rather than risk under no physical activity intervention 
(Appendix Table 2.3.2 compared with Table 2.2). Estimates from conventional pooled logistic models were qualitatively similar to these estimates, but further from the null (Appendix Table 2.3.3 compared with Appendix Table 2.3.2).

As one of our sensitivity analyses for unmeasured confounding, we conducted an alternative analysis in which physical activity and covariate data are lagged by two years. This approach assumes (1) a two year lag between physical activity and its potential effects on chronic diseases and mortality, (2) any diagnosed chronic disease existed in a subclinical state for two years, and (3) any undiagnosed chronic disease would have been diagnosed by two years. 


\begin{tabular}{|c|c|c|c|}
\hline A. Time-fixed covariates & $\begin{array}{l}\text { Functional form } \\
\text { as predictor }\end{array}$ & $\begin{array}{r}\text { Variable } \\
\text { name }\end{array}$ & Categories \\
\hline \multicolumn{4}{|c|}{ Baseline (assessed in first post-diagnostic questionnaire) } \\
\hline Age & 4 categories & $\begin{array}{l}\text { baseage_1 } \\
\text { baseage_2 } \\
\text { baseage_3 } \\
\text { baseage_4 }\end{array}$ & $\begin{array}{r}<65 \text { years } \\
65-69.9 \text { years } \\
70-74.9 \text { years } \\
\geq 75 \text { years }\end{array}$ \\
\hline Clinical stage at diagnosis & 2 categories & $\begin{array}{l}\text { stage_1 } \\
\text { stage_2 }\end{array}$ & $\begin{array}{r}\mathrm{T} 1 \\
\mathrm{~T} 2, \mathrm{~T} 3, \mathrm{~T} 4, \mathrm{~N} 1 / \mathrm{M} 0\end{array}$ \\
\hline PSA level at diagnosis & 2 categories & $\begin{array}{l}\text { psa_1 } \\
\text { psa_2 }\end{array}$ & $\begin{array}{l}<4 \mathrm{ng} / \mathrm{mL} \\
\geq 4 \mathrm{ng} / \mathrm{mL}\end{array}$ \\
\hline Gleason grade at diagnosis & 3 categories & $\begin{array}{l}\text { gleason_1 } \\
\text { gleason_2 } \\
\text { gleason_3 }\end{array}$ & $\begin{array}{r}<7 \\
7 \\
>7\end{array}$ \\
\hline Primary treatment & 3 categories & $\begin{array}{l}\text { treat_1 } \\
\text { treat_2 } \\
\text { treat_3 }\end{array}$ & $\begin{array}{r}\text { Radical prostatectomy } \\
\text { Radiation } \\
\text { Hormones, watchful } \\
\text { waiting, other }\end{array}$ \\
\hline $\begin{array}{l}\text { Parental history of myocardial } \\
\text { infarction before age } 60\end{array}$ & Indicator & fhxmi & Yes/No \\
\hline \multicolumn{4}{|c|}{ Pre-baseline (assessed in first pre-diagnostic questionnaire) } \\
\hline BMI & 4 categories & $\begin{array}{l}\text { bmi_pre_1 } \\
\text { bmi_pre_2 } \\
\text { bmi_pre_3 } \\
\text { bmi_pre_4 }\end{array}$ & $\begin{array}{r}<18.5 \mathrm{~kg} / \mathrm{m}^{2} \\
18.5-24.9 \mathrm{~kg} / \mathrm{m}^{2} \\
25.0-29.9 \mathrm{~kg} / \mathrm{m}^{2} \\
\geq 30 \mathrm{~kg} / \mathrm{m}^{2}\end{array}$ \\
\hline Vigorous physical activity & 4 categories & $\begin{array}{l}\text { vigact_pre_1 } \\
\text { vigact_pre_2 } \\
\text { vigact_pre_3 } \\
\text { vigact_pre_4 }\end{array}$ & $\begin{array}{r}<1.25 \text { hour/week } \\
1.25-2.49 \text { hours/week } \\
2.50-3.74 \text { hours/week } \\
\geq 3.75 \text { hours/week }\end{array}$ \\
\hline Moderate physical activity & 4 categories & $\begin{array}{l}\text { modact_pre_1 } \\
\text { modact_pre_2 } \\
\text { modact_pre_3 } \\
\text { modact_pre_4 }\end{array}$ & $\begin{array}{r}<2.5 \text { hours/week } \\
2.5-4.9 \text { hours/week } \\
5-7.4 \text { hours/week } \\
\geq 7.5 \text { hours/week }\end{array}$ \\
\hline Smoking history & Indicator & smkhx & Yes/No \\
\hline
\end{tabular}


Appendix Table 2.2.1 (Continued) Covariates used to model 10-year risk of all-cause mortality among men with nonmetastatic prostate cancer, Health Professionals Follow-up Study.

\begin{tabular}{|c|c|c|c|c|}
\hline B. Time-varying covariates ${ }^{1}$ & $\begin{array}{r}\text { Modeling as } \\
\text { dependent }\end{array}$ & $\begin{array}{r}\text { Variable } \\
\text { name } \\
\end{array}$ & $\begin{array}{r}\text { Functional form } \\
\text { as predictor }\end{array}$ & $\begin{array}{r}\text { Category or knot } \\
\text { locations }\end{array}$ \\
\hline Period of follow-up & Not predicted & period & 5 period indicators & $\mathrm{N} / \mathrm{A}$ \\
\hline BMI & $\begin{array}{l}\text { Linear (on } \\
\text { log scale) }\end{array}$ & bmi & 4 categories & $\begin{array}{r}18.5,25,30 \\
\mathrm{~kg} / \mathrm{m}^{2}\end{array}$ \\
\hline Vigorous physical activity & $\begin{array}{r}\text { Logistic, then } \\
\text { log-linear }\end{array}$ & vigact & $\begin{array}{l}\text { Restricted cubic } \\
\text { splines, } 3 \text { knots }\end{array}$ & $\begin{array}{r}1.25,2.5,3.75 \\
\text { hours/week }\end{array}$ \\
\hline Moderate physical activity & Linear $^{2}$ & modcat & $\begin{array}{l}\text { Restricted cubic } \\
\text { splines, } 3 \text { knots }\end{array}$ & $\begin{array}{r}2.5,5,7.5 \\
\text { hours/week }\end{array}$ \\
\hline $\begin{array}{l}\text { Development of functional } \\
\text { impairment, metastasis, } \\
\text { myocardial infarction, stroke, } \\
\text { congestive heart failure, or } \\
\text { amyotrophic lateral sclerosis }\end{array}$ & $\begin{array}{l}\text { Logistic to } \\
\text { failure }\end{array}$ & xcond & $\begin{array}{l}\text { Indicator and } \\
\text { time since switch }\end{array}$ & $\mathrm{N} / \mathrm{A}$ \\
\hline
\end{tabular}

${ }^{1}$ Time-varying covariates were assessed in all periods.

${ }^{2}$ Variables predicted by a linear model were assigned a value equal to the predicted value plus the standard error multiplied by a random number from a Normal $(0,1)$ distribution. Therefore, two subjects with the same risk factor history were not necessarily predicted to have exactly the same risk factor value at the next time point. Simulated values of continuous risk factors were truncated so that they did not fall outside of the observed range.

${ }^{3}$ Variables with many zero values were predicted in two stages. First, we fit a logistic model on an indicator that the variable is nonzero. Second, we fit a linear model for the natural log of the nonzero values. Simulated values were truncated so that they did not fall outside of the observed range.

${ }^{4}$ Variables predicted by a logistic model were assigned a value of 1 if the predicted probability was greater than a random number from a uniform distribution. After the first 1 is generated, the value is set to 1 thereafter. 
Appendix Table 2.2.2.1 Cumulative and average percent of men intervened on under strategies in Table 2.2: Estimated risk of all-cause mortality under several physical activity strategies among men with nonmetastatic prostate cancer, Health Professionals Follow-up Study. All strategies excuse men from following the recommended physical activity levels after development of functional impairment, metastasis, myocardial infarction, stroke, congestive heart failure, or amyotrophic lateral sclerosis.

\begin{tabular}{|c|c|c|}
\hline Strategy & Cumulative $\%$ intervened on ${ }^{a}$ & Average $\%$ intervened on ${ }^{b}$ \\
\hline No intervention & 0 & 0 \\
\hline \multicolumn{3}{|c|}{ Vigorous activity } \\
\hline$\geq 1.25 \mathrm{~h} /$ week & 87 & 57 \\
\hline$\geq 2.5 \mathrm{~h} /$ week & 93 & 63 \\
\hline$\geq 3.75 \mathrm{~h} /$ week & 97 & 72 \\
\hline \multicolumn{3}{|c|}{ Moderate activity } \\
\hline$\geq 2.5 \mathrm{~h} /$ week & 57 & 24 \\
\hline$\geq 5 \mathrm{~h} /$ week & 73 & 34 \\
\hline$\geq 7.5 \mathrm{~h} /$ week & 85 & 45 \\
\hline
\end{tabular}

Estimates based on the parametric g-formula adjusted for age, parental history of myocardial infarction, primary treatment, clinical stage at diagnosis, Gleason grade at diagnosis, PSA level at diagnosis, smoking history, body mass index, vigorous and moderate physical activity, and the development of functional impairment, metastasis, myocardial infarction, stroke, congestive heart failure, or amyotrophic lateral sclerosis.

${ }^{a}$ Cumulative proportion of men who would have to change their behavior in any time period over follow-up to keep adhering to the strategy.

${ }^{\mathrm{b}}$ Average proportion of men who would have to change their behavior in each 2-year period to keep adhering to the strategy. 


\begin{tabular}{|c|c|c|}
\hline \multicolumn{3}{|c|}{$\begin{array}{l}\text { Appendix Table 2.2.2.2 Cumulative and average percent of men intervened on under } \\
\text { strategies in Table 2.3: Estimated risk of all-cause mortality under several physical activity } \\
\text { strategies among men with nonmetastatic prostate cancer, Health Professionals Follow-up } \\
\text { Study. All strategies excuse men from following the recommended physical activity levels after } \\
\text { development of functional impairment, metastasis, or a serious chronic condition. }{ }^{a}\end{array}$} \\
\hline Strategy & Cumulative $\%$ intervened on ${ }^{b}$ & Average $\%$ intervened on ${ }^{c}$ \\
\hline No intervention & 0 & 0 \\
\hline \multicolumn{3}{|c|}{ Vigorous activity } \\
\hline$\geq 1.25 \mathrm{~h} /$ week & 57 & 38 \\
\hline$\geq 2.5 \mathrm{~h} /$ week & 62 & 42 \\
\hline$\geq 3.75 \mathrm{~h} /$ week & 66 & 48 \\
\hline \multicolumn{3}{|c|}{ Moderate activity } \\
\hline$\geq 2.5 \mathrm{~h} /$ week & 37 & 16 \\
\hline$\geq 5 \mathrm{~h} /$ week & 48 & 23 \\
\hline$\geq 7.5 \mathrm{~h} /$ week & 56 & 30 \\
\hline
\end{tabular}

Estimates based on the parametric g-formula adjusted for age, parental history of myocardial infarction, primary treatment, clinical stage at diagnosis, Gleason grade at diagnosis, PSA level at diagnosis, smoking history, body mass index, vigorous and moderate physical activity, and the development of functional impairment, metastasis, myocardial infarction, stroke, congestive heart failure, or amyotrophic lateral sclerosis.

${ }^{a}$ Myocardial infarction, stroke, congestive heart failure, amyotrophic lateral sclerosis, or any of the following conditions: angina pectoris, pulmonary embolism, heart rhythm disturbance, diabetes, chronic renal failure, rheumatoid arthritis, gout, ulcerative colitis or Crohn's disease, emphysema, Parkinson's disease, and multiple sclerosis.

${ }^{\mathrm{b}}$ Cumulative proportion of men who would have to change their behavior in any time period over follow-up to keep adhering to the strategy.

${ }^{\mathrm{c}}$ Average proportion of men who would have to change their behavior in each 2-year period to keep adhering to the strategy. 
Appendix Figure 1.1 Mean differences between observed and simulated values for body mass index and their $95 \%$ confidence intervals by year of follow-up. Differences were never greater than $0.04 \%$.

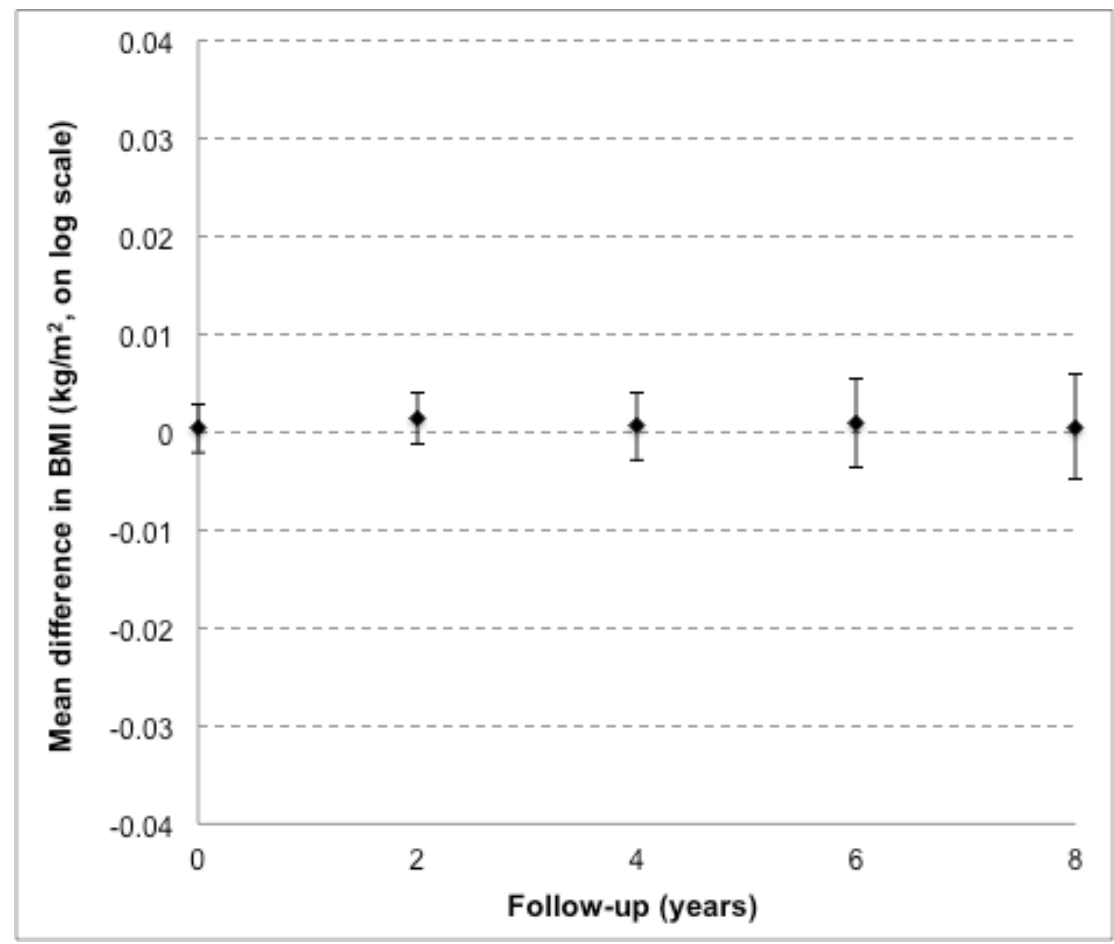

Appendix Figure 1.2: Mean differences between observed and simulated values for moderate activity and their $95 \%$ confidence intervals by year of follow-up. Differences were never greater than $16 \%$.

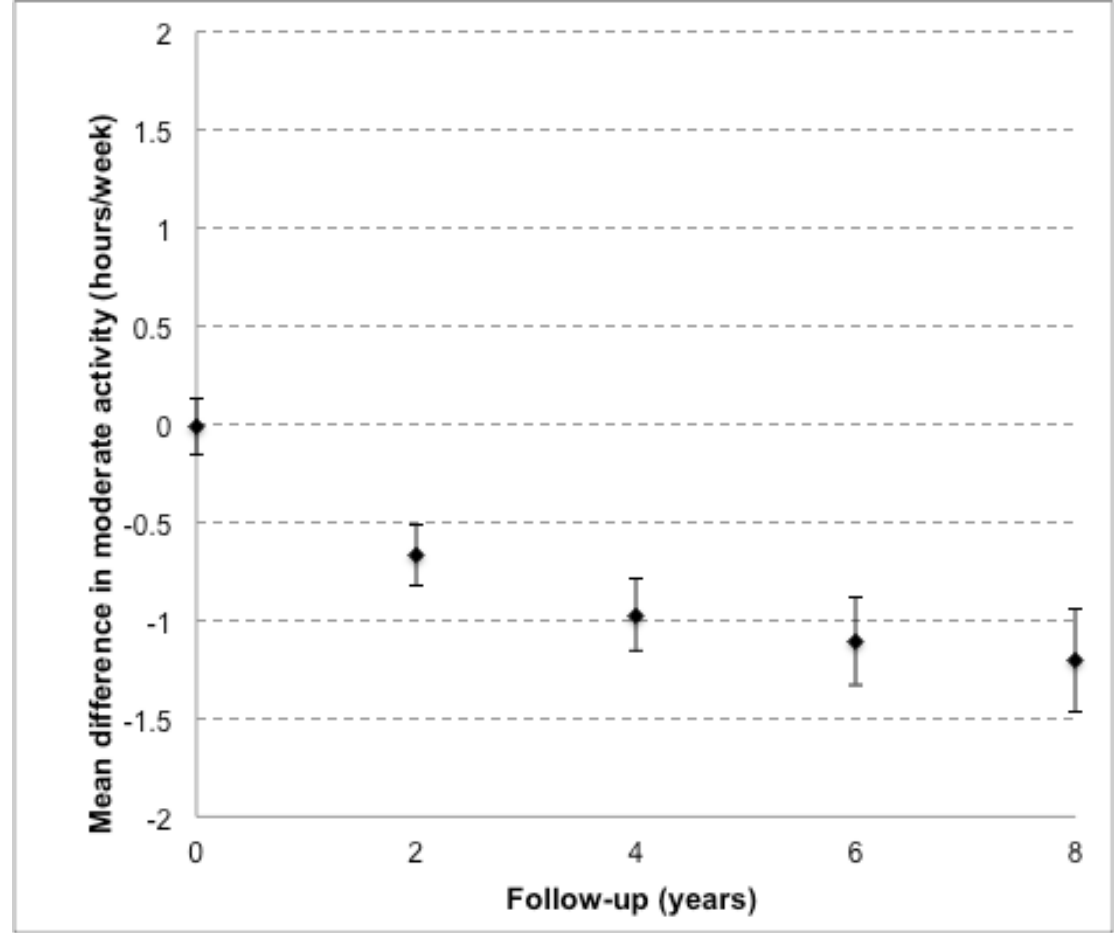


Appendix Figure 1.3: Mean differences between observed and simulated values for vigorous activity and their $95 \%$ confidence intervals by year of follow-up. Differences were never greater than $5 \%$.

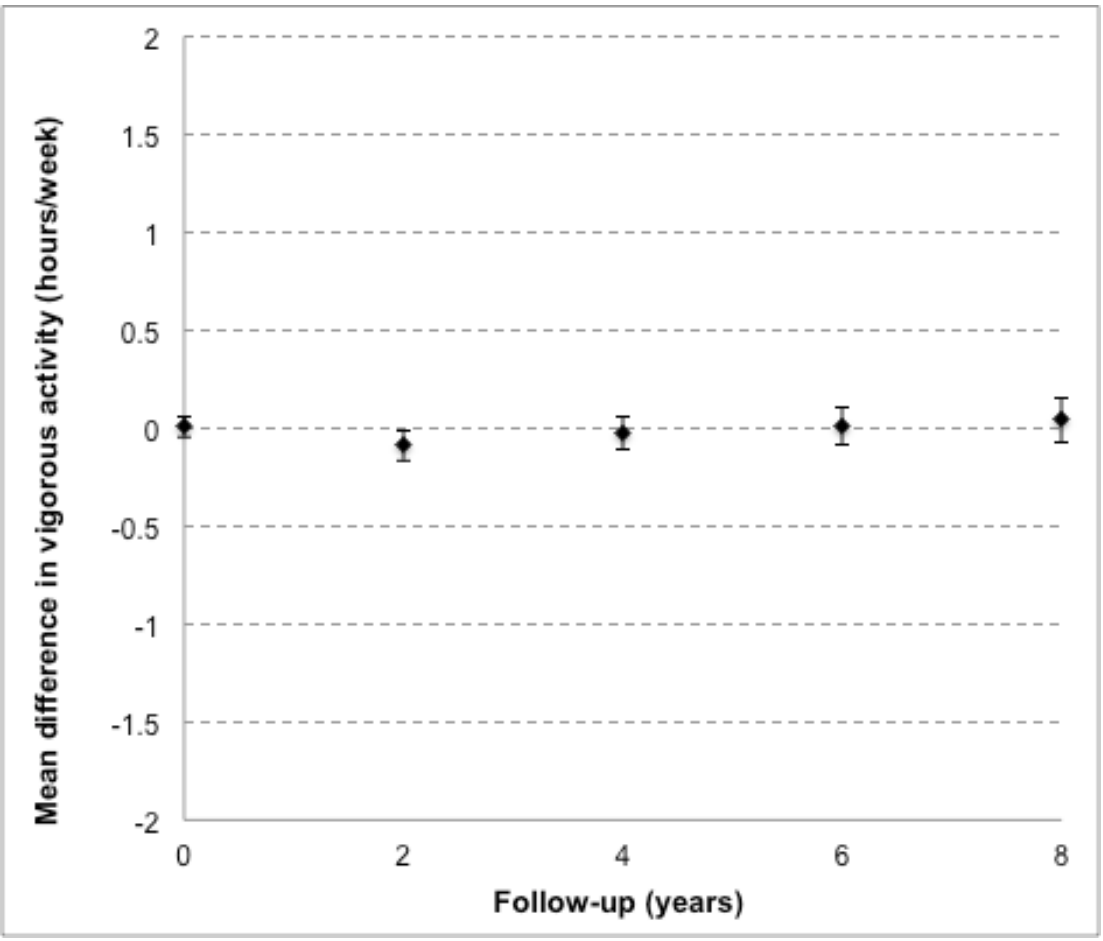

Appendix Figure 1.4: Mean differences between observed and simulated values for an indicator of conditions limiting physical ability (functional impairment, metastasis, myocardial infarction, stroke, congestive heart failure, amyotrophic lateral sclerosis) and their 95\% confidence intervals by year of follow-up. Differences were never greater than $15 \%$.

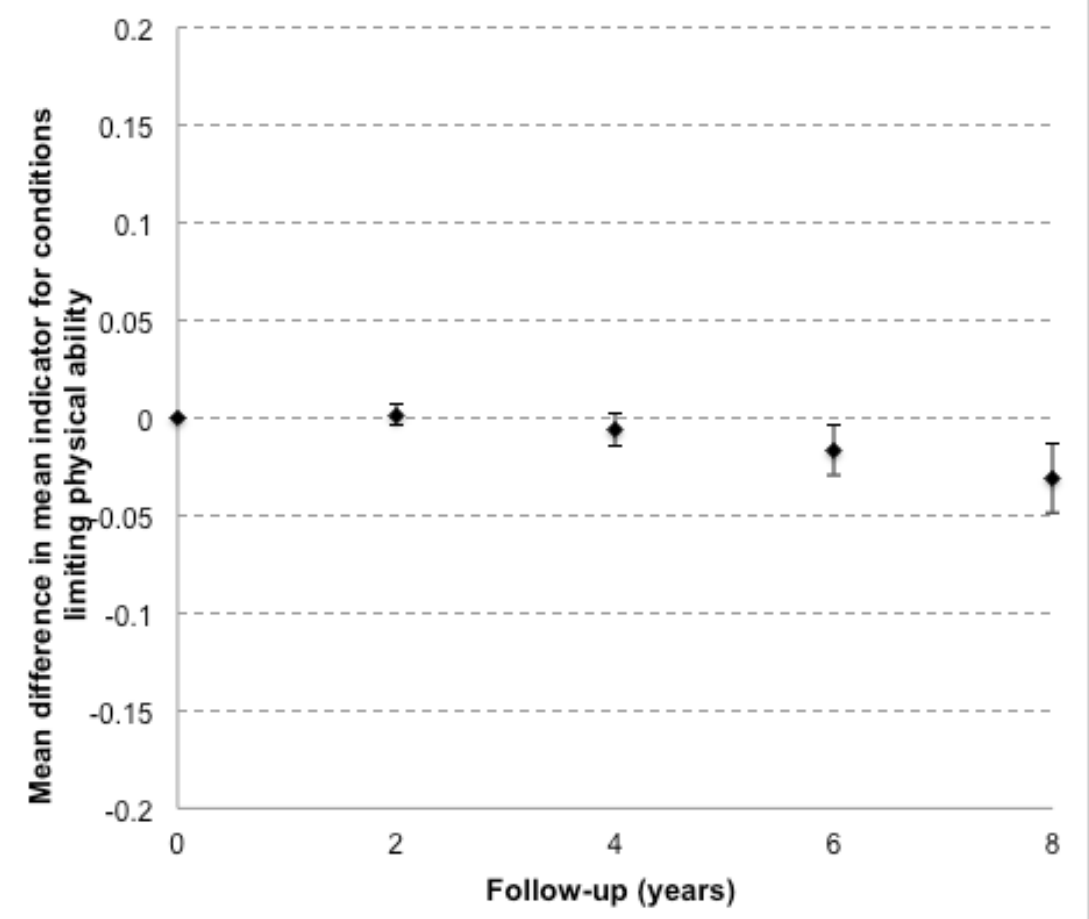


Appendix 2.3 Results from additional analyses

Appendix Table 2.3.1 Estimated survival time and time invested in physical activity over 10 years under each strategy among men with nonmetastatic prostate cancer, Health Professionals Follow-up Study.

\begin{tabular}{|c|c|c|c|c|}
\hline Strategy & $\begin{array}{r}\text { Average number } \\
\text { of days (months) } \\
\text { lived }\end{array}$ & $\begin{array}{l}\text { Average number } \\
\text { of days (months) } \\
\text { lived, difference }\end{array}$ & $\begin{array}{r}\text { Average number of } \\
\text { days (months) } \\
\text { invested in } \\
\text { physical activity }\end{array}$ & $\begin{array}{r}\text { Average number of } \\
\text { days (months) } \\
\text { invested in } \\
\text { physical activity, } \\
\text { difference }\end{array}$ \\
\hline & & & 35 (1.2) (vigorous) & \\
\hline No intervention & $3,408(113.6)$ & 0 & $180(6.0)$ (moderate) & 0 \\
\hline \multicolumn{5}{|l|}{ Vigorous activity } \\
\hline $1.25 \mathrm{~h} /$ week & 3,448 (114.9) & $40(1.3)$ & $56(1.9)$ & $21(0.7)$ \\
\hline $2.5 \mathrm{~h} /$ week & $3,479(116.0)$ & $71(2.4)$ & $81(2.7)$ & $46(1.5)$ \\
\hline $3.75 \mathrm{~h} /$ week & $3,489(116.3)$ & $81(2.7)$ & $103(3.4)$ & $67(2.2)$ \\
\hline \multicolumn{5}{|c|}{ Moderate activity } \\
\hline $2.5 \mathrm{~h} /$ week & $3,435(114.5)$ & $27(0.9)$ & $194(6.5)$ & $14(0.5)$ \\
\hline 5 h/week & $3,458(115.3)$ & $50(1.7)$ & $216(7.2)$ & $36(1.2)$ \\
\hline $7.5 \mathrm{~h} /$ week & $3,463(115.4)$ & $55(1.8)$ & $243(8.1)$ & $63(2.1)$ \\
\hline
\end{tabular}

Average number of days lived calculated as restricted mean survival time under each strategy.

Difference in average number of days lived calculated as the difference in restricted mean survival time comparing the specified strategy with no intervention.

Average number of days invested in strategy-specific (i.e. vigorous or moderate) physical activity over follow-up.

Difference in average number of days invested in physical activity calculated as the difference in average number of days invested in physical activity comparing the specified strategy with no intervention. Differences were calculated based on values before rounding. 
Appendix Table 2.3.2 Same as Table 2.2 but with low levels of physical activity as the reference: Estimated risk of all-cause mortality under several physical activity strategies among men with nonmetastatic prostate cancer, Health Professionals Follow-up Study. All strategies excuse men from following the recommended physical activity levels after development of functional impairment, metastasis, myocardial infarction, stroke, congestive heart failure, or amyotrophic lateral

sclerosis.

\begin{tabular}{|c|c|c|c|c|}
\hline Strategy & $\begin{array}{r}10 \text {-year } \\
\text { risk (\%) }\end{array}$ & $95 \% \mathrm{Cl}$ & Risk ratio & $95 \% \mathrm{Cl}$ \\
\hline \multicolumn{5}{|c|}{ Vigorous activity } \\
\hline$<1.25 \mathrm{~h} /$ week & 16.7 & $(14.6,18.8)$ & 1.0 & -- \\
\hline$\geq 1.25 \mathrm{~h} /$ week & 13.0 & $(10.9,15.4)$ & 0.78 & $(0.68,0.91)$ \\
\hline$\geq 2.5 \mathrm{~h} /$ week & 11.1 & $(8.7,14.1)$ & 0.66 & $(0.52,0.85)$ \\
\hline$\geq 3.75 \mathrm{~h} /$ week & 10.5 & $(8.0,13.5)$ & 0.62 & $(0.48,0.83)$ \\
\hline \multicolumn{5}{|c|}{ Moderate activity } \\
\hline$<2.5$ h/week & 19.8 & $(17.4,22.4)$ & 1.0 & -- \\
\hline$\geq 2.5 \mathrm{~h} /$ week & 13.9 & $(12.0,16.0)$ & 0.70 & $(0.62,0.79)$ \\
\hline$\geq 5 \mathrm{~h} /$ week & 12.6 & $(10.6,14.7)$ & 0.63 & $(0.54,0.74)$ \\
\hline$\geq 7.5 \mathrm{~h} /$ week & 12.2 & $(10.3,14.4)$ & 0.62 & $(0.51,0.72)$ \\
\hline
\end{tabular}

Estimates based on the parametric g-formula adjusted for age, parental history of myocardial infarction, primary treatment, clinical stage at diagnosis, Gleason grade at diagnosis, PSA level at diagnosis, smoking history, body mass index, vigorous and moderate physical activity, and the development of functional impairment,

metastasis, myocardial infarction, stroke, congestive heart failure, or amyotrophic lateral sclerosis.

a The observed risk was $15.4 \%$. There were 250 observed deaths among 2,299 men over 8,972 person-years of follow-up. 
Appendix Table 2.3.3 Estimated odds ratios for all-cause mortality in two-year intervals by duration of weekly physical activity cancer among men with nonmetastatic prostate cancer, Health Professionals Follow-up Study.

\begin{tabular}{|c|c|c|c|c|c|c|c|c|}
\hline & \multicolumn{4}{|c|}{ Vigorous activity (hours/week) } & \multicolumn{4}{|c|}{ Moderate activity (hours/week) } \\
\hline & $<1.25$ & 1.25 to $<2.5$ & 2.5 to $<3.75$ & $\geq 3.75$ & $<2.5$ & 2.5 to $<5$ & 5 to $<7.5$ & $\geq 7.5$ \\
\hline Cases & 201 & 15 & 17 & 17 & 125 & 41 & 30 & 54 \\
\hline Person-years & 5,706 & 841 & 1,058 & 1,367 & 2,442 & 1,763 & 1,536 & 3,231 \\
\hline Age-adjusted OR $(95 \% \mathrm{Cl})$ & 1 & $\begin{array}{r}0.61 \\
(0.36,1.04)\end{array}$ & $\begin{array}{r}0.61 \\
(0.37,1.01)\end{array}$ & $\begin{array}{r}0.51 \\
(0.30,0.84)\end{array}$ & 1 & $\begin{array}{r}0.54 \\
(0.37,0.77)\end{array}$ & $\begin{array}{r}0.46 \\
(0.30,0.69)\end{array}$ & $\begin{array}{r}0.40 \\
(0.28,0.55)\end{array}$ \\
\hline +pre-baseline physical activity & 1 & $\begin{array}{r}0.60 \\
(0.35,1.04)\end{array}$ & $\begin{array}{r}0.60 \\
(0.35,1.01)\end{array}$ & $\begin{array}{r}0.53 \\
(0.30,0.92)\end{array}$ & 1 & $\begin{array}{r}0.52 \\
(0.36,0.75)\end{array}$ & $\begin{array}{r}0.44 \\
(0.29,0.68)\end{array}$ & $\begin{array}{r}0.38 \\
(0.26,0.54)\end{array}$ \\
\hline +baseline covariates & 1 & $\begin{array}{r}0.58 \\
(0.34,1.01)\end{array}$ & $\begin{array}{r}0.62 \\
(0.37,1.04)\end{array}$ & $\begin{array}{r}0.52 \\
(0.30,0.91)\end{array}$ & 1 & $\begin{array}{r}0.51 \\
(0.35,0.74)\end{array}$ & $\begin{array}{r}0.44 \\
(0.29,0.67)\end{array}$ & $\begin{array}{r}0.39 \\
(0.27,0.56)\end{array}$ \\
\hline +simple update covariates & 1 & $\begin{array}{r}0.57 \\
(0.33,0.99)\end{array}$ & $\begin{array}{r}0.62 \\
(0.37,1.05)\end{array}$ & $\begin{array}{r}0.52 \\
(0.30,0.92)\end{array}$ & 1 & $\begin{array}{r}0.52 \\
(0.36,0.75)\end{array}$ & $\begin{array}{r}0.44 \\
(0.29,0.67)\end{array}$ & $\begin{array}{r}0.38 \\
(0.26,0.54)\end{array}$ \\
\hline +intermediate diagnoses & 1 & $\begin{array}{r}0.59 \\
(0.34,1.03)\end{array}$ & $\begin{array}{r}0.62 \\
(0.36,1.05)\end{array}$ & $\begin{array}{r}0.56 \\
(0.32,0.99)\end{array}$ & 1 & $\begin{array}{r}0.60 \\
(0.41,0.88)\end{array}$ & $\begin{array}{r}0.47 \\
(0.31,0.73)\end{array}$ & $\begin{array}{r}0.44 \\
(0.30,0.63)\end{array}$ \\
\hline
\end{tabular}

Estimates based on pooled logistic regression models updating physical activity each time period. Vigorous and moderate physical activity were mutually adjusted. To age-adjusted models (containing linear and quadratic terms for age), we sequentially added the following adjustment variables: pre-baseline physical activity, baseline covariates, simple update covariates, and intermediate diagnoses. Prebaseline physical activity included vigorous and moderate activity. Baseline covariates included parental history of myocardial infarction, primary treatment, clinical stage at diagnosis, Gleason grade at diagnosis, PSA level at diagnosis, smoking history, pre-baseline body mass index. Simple update covariates included body mass index. Intermediate diagnoses included the development of functional impairment, metastasis, myocardial infarction, stroke, congestive heart failure, or amyotrophic lateral sclerosis. 
Appendix 2.4 Results from sensitivity analyses

Appendix Table 2.4.1 Sensitivity analysis using a negative outcome control: Estimated risk of questionnaire non-response under several physical activity strategies among men with nonmetastatic prostate cancer, Health Professionals Follow-up Study. All strategies excuse men from following the recommended physical activity levels after development of functional impairment, metastasis, myocardial infarction, stroke, congestive heart failure, or amyotrophic lateral sclerosis.

\begin{tabular}{|c|c|c|c|c|c|c|c|c|}
\hline Strategy & $\begin{array}{r}10 \text {-year } \\
\text { risk (\%) }\end{array}$ & $95 \% \mathrm{Cl}$ & $\begin{array}{l}\text { Risk } \\
\text { ratio }\end{array}$ & $95 \% \mathrm{Cl}$ & $\begin{array}{r}\text { Risk } \\
\text { difference } \\
\end{array}$ & $95 \% \mathrm{Cl}$ & $\begin{array}{r}\text { Cumulative \% } \\
\text { intervened on }\end{array}$ & $\begin{array}{r}\text { Average \% } \\
\text { intervened on }\end{array}$ \\
\hline No intervention & 12.8 & $(11.2,14.7)$ & 1.0 & -- & 0 & -- & 0 & 0 \\
\hline \multicolumn{9}{|c|}{ Vigorous activity } \\
\hline$\geq 1.25 \mathrm{~h} /$ week & 13.5 & $(11.2,15.8)$ & 1.05 & $(0.93,1.17)$ & 0.7 & $(-0.8,2.1)$ & 87 & 57 \\
\hline$\geq 2.5 \mathrm{~h} /$ week & 13.5 & $(10.5,16.9)$ & 1.05 & $(0.86,1.30)$ & 0.7 & $(-1.7,3.7)$ & 93 & 63 \\
\hline$\geq 3.75$ h/week & 12.6 & $(9.7,15.9)$ & 0.98 & $(0.79,1.22)$ & -0.2 & $(-2.7,2.9)$ & 97 & 72 \\
\hline \multicolumn{9}{|c|}{ Moderate activity } \\
\hline$\geq 2.5 \mathrm{~h} /$ week & 12.3 & $(10.5,14.1)$ & 0.96 & $(0.91,0.99)$ & -0.5 & $(-1.2,-0.1)$ & 57 & 24 \\
\hline$\geq 5 \mathrm{~h} /$ week & 11.7 & $(9.9,13.8)$ & 0.92 & $(0.83,0.99)$ & -1.1 & $(-2.1,-0.2)$ & 73 & 34 \\
\hline$\geq 7.5 \mathrm{~h} /$ week & 11.8 & $(9.9,13.9)$ & 0.92 & $(0.83,0.99)$ & -1.0 & $(-2.1,-0.1)$ & 85 & 45 \\
\hline
\end{tabular}

Estimates based on the parametric g-formula adjusted for age, parental history of myocardial infarction, primary treatment, clinical stage at diagnosis, Gleason grade at diagnosis, PSA level at diagnosis, smoking history, body mass index, vigorous and moderate physical activity, and the development of metastasis, myocardial infarction, stroke, congestive heart failure, amyotrophic lateral sclerosis, or functional impairment.

a The observed risk was $12.7 \%$. There were 205 events among 2,299 men over 8,927 person-years of follow-up.

${ }^{b}$ Cumulative proportion of men who would have to change their behavior in any time period over follow-up to keep adhering to the strategy.

${ }^{\mathrm{c}}$ Average proportion of men who would have to change their behavior in each 2-year period to keep adhering to the strategy. 
Appendix Table 2.4.2 Same as Table 2.2 except assuming that the development of functional impairment, metastasis, and chronic diseases occurred 2 years before the questionnaire in which these conditions were reported. This involved excluding those developing these conditions through the end of the first period and stopping the interventions in the period before (rather than after) any of these conditions were reported to have developed over follow-up: Estimated risk of all-cause mortality under several physical activity strategies among men with nonmetastatic prostate cancer, Health Professionals Follow-up Study.

\begin{tabular}{|c|c|c|c|c|c|c|c|c|}
\hline Strategy & $\begin{array}{l}10 \text {-year } \\
\text { risk }(\%)^{a}\end{array}$ & $95 \% \mathrm{Cl}$ & $\begin{array}{l}\text { Risk } \\
\text { ratio }\end{array}$ & $95 \% \mathrm{Cl}$ & $\begin{array}{r}\text { Risk } \\
\text { difference }\end{array}$ & $95 \% \mathrm{Cl}$ & $\begin{array}{l}\text { Cumulative \% } \\
\text { intervened on }\end{array}$ & $\begin{array}{l}\text { Average \% } \\
\text { intervened on }\end{array}$ \\
\hline No intervention & 13.7 & $(12.0,15.8)$ & 1.0 & -- & 0 & -- & 0 & 0 \\
\hline \multicolumn{9}{|c|}{ Vigorous activity } \\
\hline$\geq 1.25$ h/week & 11.5 & $(9.6,13.9)$ & 0.83 & $(0.73,0.95)$ & -2.3 & $(-3.8,-0.7)$ & 86 & 56 \\
\hline$\geq 2.5 \mathrm{~h} /$ week & 9.7 & $(7.4,12.8)$ & 0.71 & $(0.55,0.88)$ & -4.0 & $(-6.2,-1.6)$ & 93 & 61 \\
\hline$\geq 3.75 \mathrm{~h} /$ week & 9.2 & $(7.0,12.4)$ & 0.67 & $(0.52,0.86)$ & -4.5 & $(-6.9,-1.9)$ & 97 & 71 \\
\hline \multicolumn{9}{|c|}{ Moderate activity } \\
\hline$\geq 2.5 \mathrm{~h} /$ week & 12.1 & $(10.4,14.2)$ & 0.88 & $(0.83,0.94)$ & -1.6 & $(-2.5,-0.8)$ & 58 & 24 \\
\hline$\geq 5 \mathrm{~h} /$ week & 11.1 & $(9.2,13.3)$ & 0.81 & $(0.71,0.90)$ & -2.7 & $(-4.0,-1.5)$ & 74 & 34 \\
\hline$\geq 7.5 \mathrm{~h} /$ week & 11.0 & $(9.0,13.1)$ & 0.80 & $(0.70,0.89)$ & -2.7 & $(-4.2,-1.6)$ & 85 & 44 \\
\hline
\end{tabular}

Estimates based on the parametric g-formula adjusted for age, parental history of myocardial infarction, primary treatment, clinical stage at diagnosis, Gleason grade at diagnosis, PSA level at diagnosis, smoking history, body mass index, vigorous and moderate physical activity, and the development of functional impairment, metastasis, myocardial infarction, stroke, congestive heart failure, or amyotrophic lateral sclerosis.

a The observed risk was 14.0\%. There were 209 observed deaths among 2,149 men over 8,431 person-years of follow-up.

${ }^{b}$ Cumulative proportion of men who would have to change their behavior in any time period over follow-up to keep adhering to the strategy.

${ }^{c}$ Average proportion of men who would have to change their behavior in each 2-year period to keep adhering to the strategy. 
Appendix Table 2.4.3 Same as Table 2.2 except that total time devoted to vigorous and moderate physical activity was kept constant: Estimated risk of all-cause mortality under several physical activity strategies among men with nonmetastatic prostate cancer, Health Professionals Follow-up Study. All strategies excuse men from following the recommended physical activity levels after development of functional impairment, metastasis, myocardial infarction, stroke, congestive heart failure, or amyotrophic lateral sclerosis.

\begin{tabular}{|c|c|c|c|c|c|c|c|c|}
\hline Strategy & $\begin{array}{r}10 \text {-year } \\
\text { risk }(\%)^{a}\end{array}$ & $95 \% \mathrm{Cl}$ & $\begin{array}{l}\text { Risk } \\
\text { ratio }\end{array}$ & $95 \% \mathrm{Cl}$ & $\begin{array}{r}\text { Risk } \\
\text { difference }\end{array}$ & $95 \% \mathrm{Cl}$ & $\begin{array}{l}\text { Cumulative \% } \\
\text { intervened on }\end{array}$ & $\begin{array}{l}\text { Average \% } \\
\text { intervened on }\end{array}$ \\
\hline No intervention & 15.4 & $(13.7,17.4)$ & 1.0 & -- & 0 & -- & 0 & 0 \\
\hline \multicolumn{9}{|c|}{ Vigorous activity } \\
\hline$\geq 1.25 \mathrm{~h} /$ week & 13.4 & $(11.4,15.9)$ & 0.87 & $(0.77,0.98)$ & -2.1 & $(-3.5,-0.3)$ & 87 & 56 \\
\hline$\geq 2.5 \mathrm{~h} /$ week & 11.9 & $(9.3,15.0)$ & 0.77 & $(0.62,0.95)$ & -3.5 & $(-6.0,-0.7)$ & 93 & 63 \\
\hline$\geq 3.75$ h/week & 11.9 & $(9.1,15.0)$ & 0.77 & $(0.59,0.96)$ & -3.6 & $(-6.2,-0.6)$ & 97 & 72 \\
\hline \multicolumn{9}{|c|}{ Moderate activity } \\
\hline$\geq 2.5 \mathrm{~h} /$ week & 14.4 & $(12.4,16.5)$ & 0.93 & $(0.87,0.97)$ & -1.1 & $(-2.1,-0.4)$ & 56 & 23 \\
\hline$\geq 5$ h/week & 13.2 & $(11.0,15.5)$ & 0.86 & $(0.76,0.92)$ & -2.2 & $(-3.7,-1.2)$ & 74 & 34 \\
\hline$\geq 7.5 \mathrm{~h} /$ week & 13.1 & $(10.8,15.6)$ & 0.85 & $(0.75,0.92)$ & -2.4 & $(-3.9,-1.2)$ & 85 & 45 \\
\hline
\end{tabular}

Estimates based on the parametric g-formula adjusted for age, parental history of myocardial infarction, primary treatment, clinical stage at diagnosis, Gleason grade at diagnosis, PSA level at diagnosis, smoking history, body mass index, vigorous and moderate physical activity, and the development of functional impairment, metastasis, myocardial infarction, stroke, congestive heart failure, or amyotrophic lateral sclerosis.

a The observed risk was $15.4 \%$. There were 250 observed deaths among 2,299 men over 8,972 person-years of follow-up.

${ }^{\mathrm{b}}$ Cumulative proportion of men who would have to change their behavior in any time period over follow-up to keep adhering to the strategy.

${ }^{\mathrm{c}}$ Average proportion of men who would have to change their behavior in each 2-year period to keep adhering to the strategy. 
Appendix Table 2.4.4 Same as Table 2.2 except that a two-year lag time was imposed: Estimated risk of all-cause mortality under several physical activity strategies among men with nonmetastatic prostate cancer, Health Professionals Follow-up Study. All strategies excuse men from following the recommended physical activity levels after development of functional impairment, metastasis, myocardial infarction, stroke, congestive heart

failure, or amyotrophic lateral sclerosis.

\begin{tabular}{|c|c|c|c|c|c|c|c|c|}
\hline Strategy & $\begin{array}{r}10 \text {-year } \\
\text { risk (\%) }\end{array}$ & $95 \% \mathrm{Cl}$ & $\begin{array}{l}\text { Risk } \\
\text { ratio }\end{array}$ & $95 \% \mathrm{Cl}$ & $\begin{array}{r}\text { Risk } \\
\text { difference }\end{array}$ & $95 \% \mathrm{Cl}$ & $\begin{array}{r}\text { Cumulative \% } \\
\text { intervened on }\end{array}$ & $\begin{array}{r}\text { Average \% } \\
\text { intervened on }\end{array}$ \\
\hline \multicolumn{9}{|c|}{ Vigorous activity } \\
\hline$\geq 2.5 \mathrm{~h} /$ week & 11.7 & $(9.7,14.3)$ & 0.75 & $(0.63,0.89)$ & -3.9 & $(-6.0,-1.7)$ & 85 & 59 \\
\hline$\geq 3.75$ h/week & 11.5 & $(9.3,14.1)$ & 0.74 & $(0.61,0.88)$ & -4.1 & $(-6.3,-1.8)$ & 90 & 68 \\
\hline \multicolumn{9}{|c|}{ Moderate activity } \\
\hline$\geq 5 \mathrm{~h} /$ week & 14.0 & $(11.6,16.0)$ & 0.89 & $(0.82,0.96)$ & -1.7 & $(-2.9,-0.5)$ & 64 & 31 \\
\hline$\geq 7.5 \mathrm{~h} /$ week & 13.8 & $(11.3,15.9)$ & 0.89 & $(0.80,0.96)$ & -1.8 & $(-3.1,-0.7)$ & 76 & 42 \\
\hline
\end{tabular}

Estimates based on the parametric g-formula adjusted for age, parental history of myocardial infarction, primary treatment, clinical stage at diagnosis, Gleason grade at diagnosis, PSA level at diagnosis, smoking history, body mass index, vigorous and moderate physical activity, and the development of functional impairment, metastasis, myocardial infarction, stroke, congestive heart failure, or amyotrophic lateral sclerosis.

a The observed risk was 15.4\%. There were 250 observed deaths among 2,299 men over 8,972 person-years of follow-up.

${ }^{\mathrm{b}}$ Cumulative proportion of men who would have to change their behavior in any time period over follow-up to keep adhering to the strategy.

${ }^{c}$ Average proportion of men who would have to change their behavior in each 2-year period to keep adhering to the strategy. 


\begin{tabular}{|c|c|c|c|c|c|}
\hline vigact_pre_1 & 1 & 0.1136 & 0.2333 & 0.2372 & 0.6263 \\
\hline vigact_pre_2 & 1 & -0.0524 & 0.3215 & 0.0266 & 0.8706 \\
\hline vigact_pre_3 & 1 & 0.3216 & 0.2661 & 1.4613 & 0.2267 \\
\hline modact_pre_1 & 1 & -0.2790 & 0.1871 & 2.2227 & 0.1360 \\
\hline modact_pre_2 & 1 & 0.0895 & 0.1929 & 0.2153 & 0.6427 \\
\hline modact pre 3 & 1 & -0.3047 & 0.2209 & 1.9030 & 0.1677 \\
\hline fhxmi - & 1 & -0.4512 & 0.2314 & 3.8022 & 0.0512 \\
\hline period_1 & 1 & -1.9668 & 0.2933 & 44.9689 & $<.0001$ \\
\hline period_2 & 1 & -1.0941 & 0.2092 & 27.3642 & $<.0001$ \\
\hline period-3 & 1 & -0.7179 & 0.1933 & 13.7946 & 0.0002 \\
\hline period 4 & 1 & -0.6624 & 0.1952 & 11.5154 & 0.0007 \\
\hline xcond & 1 & 1.3141 & 0.2703 & 23.6344 & $<.0001$ \\
\hline tsxcond inter & 1 & -0.1149 & 0.1256 & 0.8378 & 0.3600 \\
\hline modact & 1 & -0.2250 & 0.0428 & 27.5893 & $<.0001$ \\
\hline modact spl1 & 1 & 0.1548 & 0.0327 & 22.3509 & $<.0001$ \\
\hline bmi $1^{-1}$ & 1 & 1.8081 & 0.6642 & 7.4107 & 0.0065 \\
\hline bmi-2 & 1 & 0.7712 & 0.3792 & 4.1365 & 0.0420 \\
\hline bmi_3 & 1 & 0.2690 & 0.3557 & 0.5718 & 0.4496 \\
\hline vigāet & 1 & -0.2727 & 0.0983 & 7.7001 & 0.0055 \\
\hline vigact_spl1 & 1 & 0.1708 & 0.0924 & 3.4156 & 0.0646 \\
\hline
\end{tabular}

Model 2 Development of conditions limiting physical activity model (composite of functional impairment, metastasis, myocardial infarction, stroke, congestive heart failure, or amyotrophic lateral sclerosis)

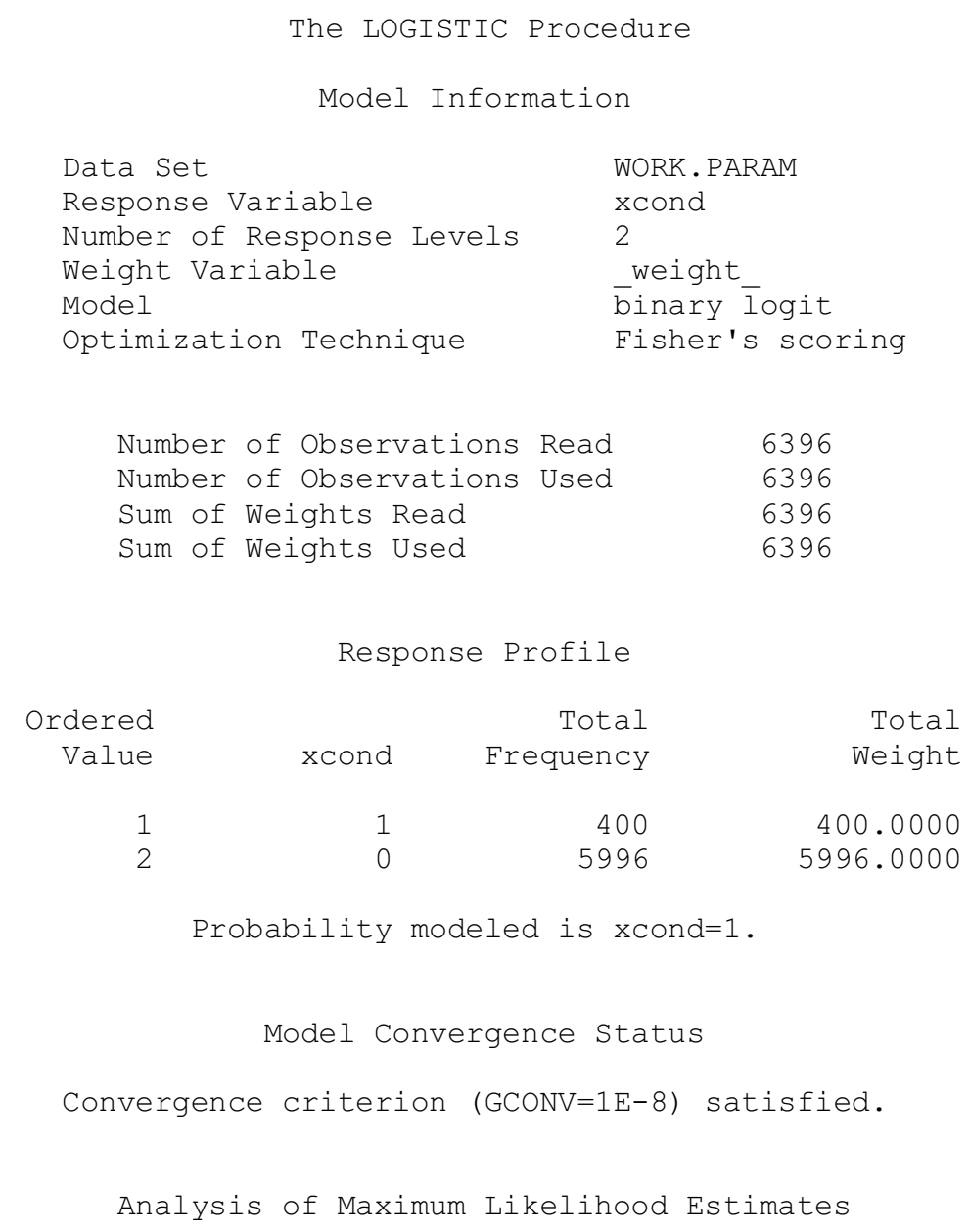




\begin{tabular}{|c|c|c|c|c|c|c|}
\hline Parameter & $\mathrm{DF}$ & Estimate & $\begin{array}{r}\text { Standard } \\
\text { Error }\end{array}$ & $\begin{array}{r}\text { Wald } \\
\text { Chi-Square }\end{array}$ & $\operatorname{Pr}$ & $>$ ChiSq \\
\hline Intercept & 1 & -0.0539 & 0.3834 & 0.0198 & & 0.8881 \\
\hline baseage_1 & 1 & -1.2596 & 0.1870 & 45.3840 & & $<.0001$ \\
\hline baseage 2 & 1 & -0.5674 & 0.1555 & 13.3161 & & 0.0003 \\
\hline baseage_3 & 1 & -0.3398 & 0.1379 & 6.0743 & & 0.0137 \\
\hline $\operatorname{smkhx}-$ & 1 & 0.0342 & 0.1082 & 0.1001 & & 0.7517 \\
\hline treat 1 & 1 & -0.5107 & 0.1838 & 7.7207 & & 0.0055 \\
\hline treat 2 & 1 & -0.1951 & 0.1670 & 1.3642 & & 0.2428 \\
\hline stage_1 & 1 & -0.2366 & 0.1162 & 4.1433 & & 0.0418 \\
\hline psa_1 & 1 & -0.3521 & 0.1759 & 4.0052 & & 0.0454 \\
\hline gleason_1 & 1 & -0.6022 & 0.1659 & 13.1855 & & 0.0003 \\
\hline gleason 2 & 1 & -0.3454 & 0.1784 & 3.7464 & & 0.0529 \\
\hline bmi_pre_-1 & 1 & -1.5383 & 1.1149 & 1.9038 & & 0.1677 \\
\hline bmi_pre_2 & 1 & -0.0499 & 0.2744 & 0.0331 & & 0.8556 \\
\hline bmi_pre_3 & 1 & -0.1727 & 0.2348 & 0.5411 & & 0.4620 \\
\hline vigact_pre_1 & 1 & -0.0748 & 0.1852 & 0.1633 & & 0.6861 \\
\hline vigact_pre_2 & 1 & -0.00800 & 0.2359 & 0.0011 & & 0.9729 \\
\hline vigact pre 3 & 1 & 0.1594 & 0.2034 & 0.6137 & & 0.4334 \\
\hline modact_pre_1 & 1 & 0.1080 & 0.1544 & 0.4888 & & 0.4845 \\
\hline modact_pre_2 & 1 & 0.2414 & 0.1619 & 2.2237 & & 0.1359 \\
\hline modact_pre_3 & 1 & 0.0816 & 0.1659 & 0.2421 & & 0.6227 \\
\hline fhxmi & 1 & 0.2013 & 0.1538 & 1.7128 & & 0.1906 \\
\hline period 1 & 0 & 0 & . & . & & . \\
\hline period_2 & 1 & -0.3085 & 0.1547 & 3.9758 & & 0.0462 \\
\hline period 3 & 1 & -0.3899 & 0.1631 & 5.7158 & & 0.0168 \\
\hline period_4 & 1 & -0.3082 & 0.1736 & 3.1541 & & 0.0757 \\
\hline modact 11 & 1 & -0.0839 & 0.0360 & 5.4254 & & 0.0198 \\
\hline modact_l1_spl1 & 1 & 0.0492 & 0.0272 & 3.2752 & & 0.0703 \\
\hline bmi $11^{-} 1$ & 1 & 0.6708 & 0.6371 & 1.1086 & & 0.2924 \\
\hline bmi_l1_2 & 1 & -0.6886 & 0.2674 & 6.6323 & & 0.0100 \\
\hline bmi-11-3 & 1 & -0.3326 & 0.2242 & 2.2013 & & 0.1379 \\
\hline vigāct_l1 & 1 & -0.1384 & 0.0718 & 3.7121 & & 0.0540 \\
\hline vigact_l1_spl1 & 1 & 0.0617 & 0.0661 & 0.8690 & & 0.3512 \\
\hline
\end{tabular}

\section{Model 3 Moderate activity model}

The REG Procedure Model: MODEL1 Dependent Variable: modact Number of Observations Read Number of Observations Used
6820

6820

$\begin{array}{lrll}\text { Root MSE } & 6.58707 & \text { R-Square } & 0.3772 \\ \text { Dependent Mean } & 7.65784 & \text { Adj R-Sq } & 0.3743 \\ \text { Coeff Var } & 86.01741 & & \end{array}$

\begin{tabular}{|c|c|c|c|c|c|}
\hline Variable & $\mathrm{DF}$ & $\begin{array}{c}\text { Parameter } \\
\text { Estimate }\end{array}$ & $\begin{array}{r}\text { Standard } \\
\text { Error }\end{array}$ & t Value & $\operatorname{Pr}>|t|$ \\
\hline Intercept & 1 & 2.69012 & 0.66876 & 4.02 & $<.0001$ \\
\hline baseage_1 & 1 & 0.85723 & 0.26537 & 3.23 & 0.0012 \\
\hline baseage 2 & 1 & 1.05468 & 0.25775 & 4.09 & $<.0001$ \\
\hline
\end{tabular}




\begin{tabular}{|c|c|c|c|c|c|}
\hline baseage 3 & 1 & 0.64540 & 0.24380 & 2.65 & 0.0081 \\
\hline $\operatorname{smkhx}$ & 1 & -0.06564 & 0.16264 & -0.40 & 0.6865 \\
\hline treat 1 & 1 & 0.10728 & 0.30874 & 0.35 & 0.7283 \\
\hline treat 2 & 1 & -0.26481 & 0.30093 & -0.88 & 0.3789 \\
\hline stage_1 & 1 & 0.04409 & 0.18358 & 0.24 & 0.8102 \\
\hline psa_1 & 1 & 0.08472 & 0.23860 & 0.36 & 0.7225 \\
\hline gleāson_1 & 1 & 0.33983 & 0.31133 & 1.09 & 0.2751 \\
\hline gleason 2 & 1 & 0.40371 & 0.33265 & 1.21 & 0.2249 \\
\hline bmi_pre 1 & 1 & -2.37861 & 1.35111 & -1.76 & 0.0784 \\
\hline bmi_pre_2 & 1 & -0.38232 & 0.44223 & -0.86 & 0.3873 \\
\hline bmi pre 3 & 1 & -0.14650 & 0.38320 & -0.38 & 0.7022 \\
\hline vigact pre 1 & 1 & -0.24552 & 0.26788 & -0.92 & 0.3594 \\
\hline vigact pre 2 & 1 & -0.49579 & 0.33759 & -1.47 & 0.1420 \\
\hline vigact pre 3 & 1 & 0.01401 & 0.29942 & 0.05 & 0.9627 \\
\hline modact_pre_1 & 1 & -2.72369 & 0.23121 & -11.78 & $<.0001$ \\
\hline modact pre 2 & 1 & -2.37572 & 0.24071 & -9.87 & $<.0001$ \\
\hline modact_pre_3 & 1 & -1.40199 & 0.24593 & -5.70 & $<.0001$ \\
\hline fhxmi & 1 & 0.43595 & 0.24342 & 1.79 & 0.0733 \\
\hline period 1 & 0 & 0 & . & . & . \\
\hline period_2 & 1 & 0.72158 & 0.24967 & 2.89 & 0.0039 \\
\hline period_3 & 1 & 0.71701 & 0.25250 & 2.84 & 0.0045 \\
\hline period 4 & 1 & 0.29881 & 0.26149 & 1.14 & 0.2532 \\
\hline$x^{\prime}$ ond - & 1 & -0.78575 & 0.51009 & -1.54 & 0.1235 \\
\hline tsxcond inter & 1 & 0.08094 & 0.24691 & 0.33 & 0.7431 \\
\hline modact $\bar{l} 1$ & 1 & 0.80444 & 0.05676 & 14.17 & $<.0001$ \\
\hline modact ${ }^{-} 11$ spl1 & 1 & -0.19971 & 0.04197 & -4.76 & $<.0001$ \\
\hline bmi $11^{-}$ & 1 & 4.32035 & 1.37096 & 3.15 & 0.0016 \\
\hline bmi 112 & 1 & 0.84932 & 0.42325 & 2.01 & 0.0448 \\
\hline bmi_11_3 & 1 & 0.63631 & 0.36352 & 1.75 & 0.0801 \\
\hline vigàct 11 & 1 & -0.10662 & 0.10294 & -1.04 & 0.3004 \\
\hline vigact 11 spll & 1 & 0.07749 & 0.08853 & 0.88 & 0.3814 \\
\hline
\end{tabular}

\section{Model $4 \mathrm{BMI}$ model}

The REG Procedure Model: MODEL1 Dependent Variable: bmi

Number of Observations Read Number of Observations Used
6820

6820
Root MSE

Dependent Mean

Coeff Var

\begin{abstract}
0.06711
3.24274

2.06948
\end{abstract}

R-Square

Adj R-Sq
0.7217

0.7203

Parameter Estimates

Variable
Intercept
baseage_1
baseage_2
baseage_3
smkhx
treat_1
treat_2
stage_1

$\begin{array}{cr}\text { DF } & \begin{array}{r}\text { Parameter } \\ \text { Estimate }\end{array} \\ 1 & 3.46142 \\ 1 & 0.01699 \\ 1 & 0.01693 \\ 1 & 0.00810 \\ 1 & 0.00309 \\ 1 & 0.00411 \\ 1 & 0.00132 \\ 1 & -0.00279\end{array}$

Standard Error

t Value

$\operatorname{Pr}>|t|$
0.00687
0.00271
0.00263
0.00249
0.00166
0.00315
0.00307
0.00187
503.79
6.27
6.43
3.26
1.86
1.31
0.43
$-1.49$

$<.0001$

$<.0001$

$<.0001$

0.0011

0.0625

0.1913

0.6675

0.1354 


\begin{tabular}{|c|c|c|c|c|c|}
\hline psa_1 & 1 & -0.00291 & 0.00243 & -1.20 & 0.2307 \\
\hline gleason_1 & 1 & -0.00258 & 0.00317 & -0.81 & 0.4153 \\
\hline gleason_2 & 1 & -0.00532 & 0.00339 & -1.57 & 0.1166 \\
\hline bmi_pre_1 & 1 & -0.13960 & 0.01377 & -10.14 & $<.0001$ \\
\hline bmi_pre_2 & 1 & -0.11586 & 0.00451 & -25.71 & $<.0001$ \\
\hline bmi pre 3 & 1 & -0.05476 & 0.00390 & -14.03 & $<.0001$ \\
\hline vigäct_p̄e_e_1 & 1 & 0.00198 & 0.00273 & 0.73 & 0.4674 \\
\hline vigact_pre_2 & 1 & 0.00270 & 0.00344 & 0.79 & 0.4320 \\
\hline vigact_pre_3 & 1 & 0.00167 & 0.00305 & 0.55 & 0.5844 \\
\hline modact_pre_1 & 1 & 0.00249 & 0.00239 & 1.04 & 0.2967 \\
\hline modact_pre_2 & 1 & 0.00261 & 0.00247 & 1.06 & 0.2904 \\
\hline modact_pre_3 & 1 & 0.00085917 & 0.00251 & 0.34 & 0.7323 \\
\hline fhxmi - & 1 & -0.00353 & 0.00248 & -1.42 & 0.1547 \\
\hline period_1 & 0 & 0 & . & . & • \\
\hline period_2 & 1 & 0.00642 & 0.00255 & 2.52 & 0.0117 \\
\hline period 3 & 1 & 0.00291 & 0.00258 & 1.13 & 0.2584 \\
\hline period 4 & 1 & 0.00144 & 0.00266 & 0.54 & 0.5893 \\
\hline xcond & 1 & -0.00474 & 0.00521 & -0.91 & 0.3626 \\
\hline tsxcond inter & 1 & 0.00171 & 0.00252 & 0.68 & 0.4977 \\
\hline modact_ $\overline{1} 1$ & 1 & 0.00020698 & 0.00064776 & 0.32 & 0.7493 \\
\hline modact_11_spl1 & 1 & -0.00006509 & 0.00047249 & -0.14 & 0.8904 \\
\hline bmi_11-1 & 1 & -0.38170 & 0.01398 & -27.31 & $<.0001$ \\
\hline bmi_l1- 2 & 1 & -0.22560 & 0.00431 & -52.30 & $<.0001$ \\
\hline bmi-11-3 & 1 & -0.12555 & 0.00370 & -33.89 & $<.0001$ \\
\hline vigāct 11 & 1 & -0.00171 & 0.00105 & -1.63 & 0.1027 \\
\hline vigact_l1_spl1 & 1 & 0.00096989 & 0.00090280 & 1.07 & 0.2827 \\
\hline modact & 1 & -0.00045448 & 0.00062425 & -0.73 & 0.4666 \\
\hline modact spl1 & 1 & 0.00000458 & 0.00045730 & 0.01 & 0.9920 \\
\hline
\end{tabular}

\section{Model 5 Vigorous activity models}

The LOGISTIC Procedure

Model Information

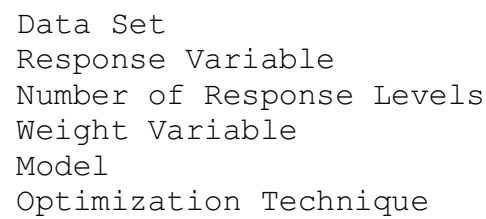

6820

6820

6820

6820

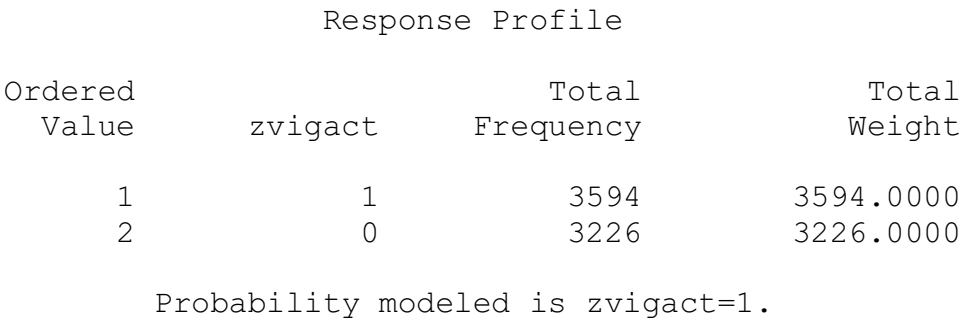

Model Convergence Status 
Convergence criterion (GCONV=1E-8) satisfied.

Analysis of Maximum Likelihood Estimates

Parameter
Intercept
baseage_1
baseage_2
baseage_3
smkhx
treat_1
treat_2
stage_1
psa_1
gleason_1
gleason_2
bmi_pre_1
bmi_pre_2
bmi_pre_3
vigact_pre_1
vigact_pre_2
vigact_pre_3
modact_pre_1
modact_pre_2
modact_pre_3
fhxmi
period_1
period_2
period_3
period_4
xcond
tsxcond_inter
modact_11
modact_11_spl1
bmi_11_1
bmi_l1_2
bmi_l1_3
vigact_11
vigact_11_spl1
modact
modact_spl1
bmi_1
bmi_2
bmi_3

\begin{tabular}{|c|c|c|c|c|c|}
\hline $\mathrm{DF}$ & Estimate & $\begin{array}{r}\text { Standard } \\
\text { Error }\end{array}$ & $\begin{array}{r}\text { Wald } \\
\text { Chi-Square }\end{array}$ & $\operatorname{Pr}$ & $>$ ChiSq \\
\hline 1 & -0.8979 & 0.2548 & 12.4194 & & 0.0004 \\
\hline 1 & 0.1565 & 0.1006 & 2.4190 & & 0.1199 \\
\hline 1 & 0.0774 & 0.0974 & 0.6321 & & 0.4266 \\
\hline 1 & -0.0756 & 0.0918 & 0.6779 & & 0.4103 \\
\hline 1 & -0.0188 & 0.0617 & 0.0927 & & 0.7608 \\
\hline 1 & -0.00515 & 0.1156 & 0.0020 & & 0.9645 \\
\hline 1 & -0.0266 & 0.1123 & 0.0561 & & 0.8128 \\
\hline 1 & -0.0332 & 0.0698 & 0.2260 & & 0.6345 \\
\hline 1 & 0.2505 & 0.0903 & 7.7006 & & 0.0055 \\
\hline 1 & -0.0630 & 0.1171 & 0.2898 & & 0.5904 \\
\hline 1 & -0.1946 & 0.1255 & 2.4032 & & 0.1211 \\
\hline 1 & -0.2860 & 0.5337 & 0.2871 & & 0.5921 \\
\hline 1 & 0.1477 & 0.1717 & 0.7401 & & 0.3896 \\
\hline 1 & 0.0553 & 0.1474 & 0.1408 & & 0.7075 \\
\hline 1 & -0.7733 & 0.1074 & 51.8625 & & $<.0001$ \\
\hline 1 & -0.1523 & 0.1354 & 1.2659 & & 0.2605 \\
\hline 1 & -0.1665 & 0.1286 & 1.6761 & & 0.1954 \\
\hline 1 & 0.00604 & 0.0886 & 0.0047 & & 0.9456 \\
\hline 1 & 0.1338 & 0.0924 & 2.0959 & & 0.1477 \\
\hline 1 & 0.0476 & 0.0934 & 0.2594 & & 0.6105 \\
\hline 1 & 0.00232 & 0.0921 & 0.0006 & & 0.9799 \\
\hline 0 & 0 & & • & & • \\
\hline 1 & 0.2977 & 0.0950 & 9.8205 & & 0.0017 \\
\hline 1 & 0.2448 & 0.0956 & 6.5549 & & 0.0105 \\
\hline 1 & 0.1821 & 0.0988 & 3.3964 & & 0.0653 \\
\hline 1 & -0.3196 & 0.1911 & 2.7962 & & 0.0945 \\
\hline 1 & 0.1810 & 0.0917 & 3.8987 & & 0.0483 \\
\hline 1 & -0.0380 & 0.0239 & 2.5280 & & 0.1118 \\
\hline 1 & 0.0157 & 0.0175 & 0.8104 & & 0.3680 \\
\hline 1 & 1.4904 & 0.6114 & 5.9429 & & 0.0148 \\
\hline 1 & 0.1136 & 0.2023 & 0.3154 & & 0.5744 \\
\hline 1 & -0.0419 & 0.1718 & 0.0594 & & 0.8075 \\
\hline 1 & 1.2295 & 0.0415 & 876.5002 & & $<.0001$ \\
\hline 1 & -0.8123 & 0.0396 & 420.3715 & & $<.0001$ \\
\hline 1 & 0.0509 & 0.0231 & 4.8470 & & 0.0277 \\
\hline 1 & -0.0337 & 0.0169 & 3.9518 & & 0.0468 \\
\hline 1 & -0.0613 & 0.5185 & 0.0140 & & 0.9058 \\
\hline 1 & 0.2039 & 0.1955 & 1.0883 & & 0.2969 \\
\hline 1 & 0.2138 & 0.1683 & 1.6138 & & 0.2040 \\
\hline
\end{tabular}




\author{
The REG Procedure \\ Model: MODEL1 \\ Dependent Variable: lvigact
}

Number of Observations Read 3594

Number of Observations Used 3594

$\begin{array}{lccc}\text { Root MSE } & 0.93504 & \text { R-Square } & 0.3856 \\ \text { Dependent Mean } & 0.57080 & \text { Adj R-Sq } & 0.3792 \\ \text { Coeff Var } & 163.81224 & & \end{array}$

\begin{tabular}{|c|c|c|c|c|c|}
\hline Variable & $\mathrm{DF}$ & $\begin{array}{r}\text { Parameter } \\
\text { Estimate }\end{array}$ & $\begin{array}{r}\text { Standard } \\
\text { Error }\end{array}$ & t Value & $\operatorname{Pr}>|t|$ \\
\hline Intercept & 1 & 0.01801 & 0.13927 & 0.13 & 0.8971 \\
\hline baseage_1 & 1 & 0.05323 & 0.05262 & 1.01 & 0.3118 \\
\hline baseage_2 & 1 & -0.06046 & 0.05137 & -1.18 & 0.2393 \\
\hline baseage_3 & 1 & -0.08028 & 0.04942 & -1.62 & 0.1044 \\
\hline smkhx & 1 & -0.04550 & 0.03186 & -1.43 & 0.1534 \\
\hline treat_1 & 1 & 0.01993 & 0.06121 & 0.33 & 0.7447 \\
\hline treat_2 & 1 & 0.03587 & 0.06006 & 0.60 & 0.5504 \\
\hline stage_1 & 1 & -0.03920 & 0.03606 & -1.09 & 0.2772 \\
\hline $\mathrm{psa}^{-}{ }^{-}$ & 1 & -0.02066 & 0.04506 & -0.46 & 0.6466 \\
\hline gleason_1 & 1 & -0.03649 & 0.06097 & -0.60 & 0.5495 \\
\hline gleason_2 & 1 & -0.03803 & 0.06581 & -0.58 & 0.5634 \\
\hline bmi_pre_1 & 1 & 0.65415 & 0.26096 & 2.51 & 0.0122 \\
\hline bmi_pre_2 & 1 & 0.07576 & 0.09952 & 0.76 & 0.4466 \\
\hline bmi_pre_3 & 1 & -0.09356 & 0.08887 & -1.05 & 0.2925 \\
\hline vigäct_p̄ere_1 & 1 & -0.54831 & 0.04670 & -11.74 & $<.0001$ \\
\hline vigact_pre_2 & 1 & -0.43617 & 0.05714 & -7.63 & $<.0001$ \\
\hline vigact_pre_3 & 1 & -0.25045 & 0.04858 & -5.16 & $<.0001$ \\
\hline modact_pre_1 & 1 & 0.05254 & 0.04731 & 1.11 & 0.2668 \\
\hline modact_pre_2 & 1 & 0.07600 & 0.04718 & 1.61 & 0.1073 \\
\hline modact_pre-3 & 1 & 0.06920 & 0.04885 & 1.42 & 0.1567 \\
\hline fhxmi & 1 & -0.04421 & 0.04746 & -0.93 & 0.3517 \\
\hline period_1 & 0 & 0 & . & . & . \\
\hline period_2 & 1 & 0.00246 & 0.04991 & 0.05 & 0.9607 \\
\hline period_3 & 1 & 0.00178 & 0.05066 & 0.04 & 0.9719 \\
\hline period_4 & 1 & -0.01600 & 0.05274 & -0.30 & 0.7617 \\
\hline xcond & 1 & 0.10218 & 0.10863 & 0.94 & 0.3470 \\
\hline tsxcond_inter & 1 & -0.07787 & 0.05163 & -1.51 & 0.1316 \\
\hline modact_ $\overline{1} 1$ & 1 & -0.04593 & 0.01291 & -3.56 & 0.0004 \\
\hline modact_l1_spl1 & 1 & 0.02445 & 0.00940 & 2.60 & 0.0093 \\
\hline bmi_11_1 & 1 & -1.14109 & 0.28188 & -4.05 & $<.0001$ \\
\hline bmi-11-2 & 1 & -0.25861 & 0.12082 & -2.14 & 0.0324 \\
\hline bmi_l1_3 & 1 & -0.16659 & 0.10718 & -1.55 & 0.1202 \\
\hline vigāct_l1 & 1 & 0.39058 & 0.02040 & 19.15 & $<.0001$ \\
\hline vigact_l1_spl1 & 1 & -0.19963 & 0.01638 & -12.19 & $<.0001$ \\
\hline modact ${ }^{-}-$ & 1 & 0.08140 & 0.01253 & 6.50 & $<.0001$ \\
\hline modact_spl1 & 1 & -0.04302 & 0.00915 & -4.70 & $<.0001$ \\
\hline bmi_1 & 1 & -0.22004 & 0.26131 & -0.84 & 0.3998 \\
\hline bmi-2 & 1 & 0.23400 & 0.11383 & 2.06 & 0.0399 \\
\hline bmi_3 & 1 & 0.29331 & 0.10126 & 2.90 & 0.0038 \\
\hline
\end{tabular}




\section{Appendix 3.1}

Appendix Figure 2 Flowchart of metabolite selection and detail on included metabolites

Appendix Figure 3 Flowchart of participant selection ............................ 115

\section{Appendix 3.2}

Appendix Figure 4 Overlap in identified metabolites across adiposity measures

Appendix Figure 5 Correlation among metabolites associated with BMI ..... 117

Appendix Figure 6 Correlation among metabolites associated with waist circumference

Appendix Figure 7 Correlation among metabolites associated with fat mass

Appendix Table 3 Metabolites associated with adiposity measures among men with data on all three adiposity measures

\section{Appendix 3.3}

Lasso models used for predicting adiposity using associated metabolites .... 125

\section{Appendix 3.4 - Results from additional analyses}

Appendix Figure 8-10 Alternate specification of BMI-metabolic groups 128 Appendix Figure 11-13 Alternate specification of waist circumferencemetabolic groups

Appendix Figure 14-16 Alternate specification of derived fat massmetabolic groups 


\section{Appendix 3.1}

Appendix Figure 2 Flowchart of metabolite selection

ALS nested case-control study

C8-positive method (polar and non-polar lipids)

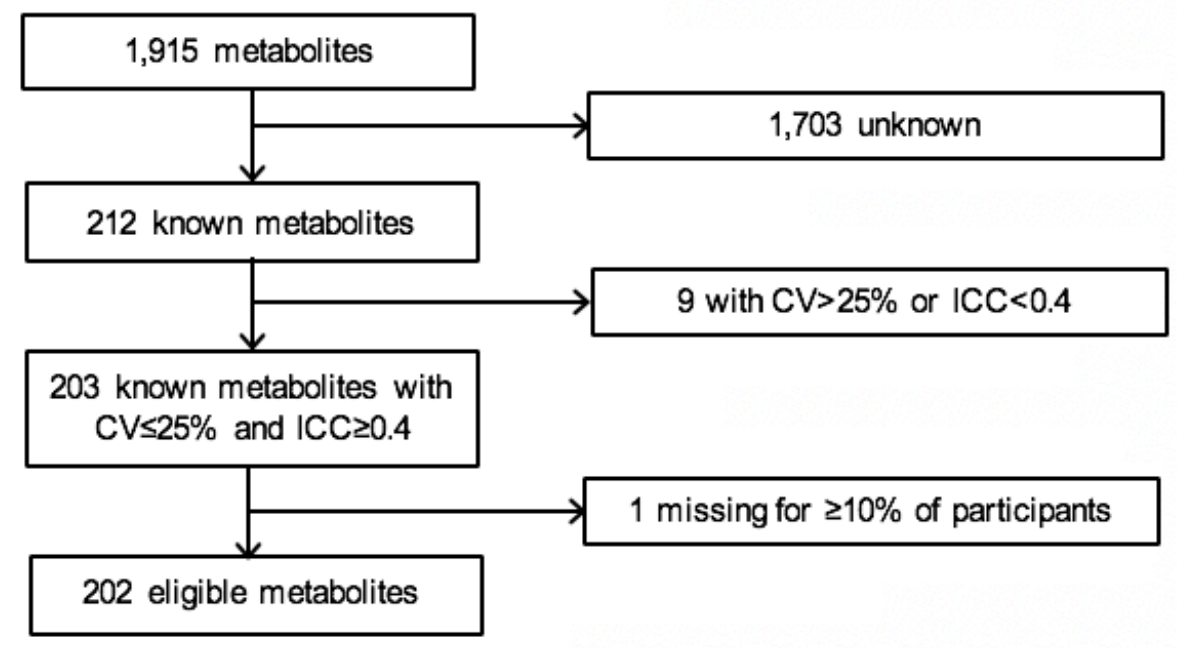

HILIC-positive method (amino acids, dipeptides, and other cationic metabolites)

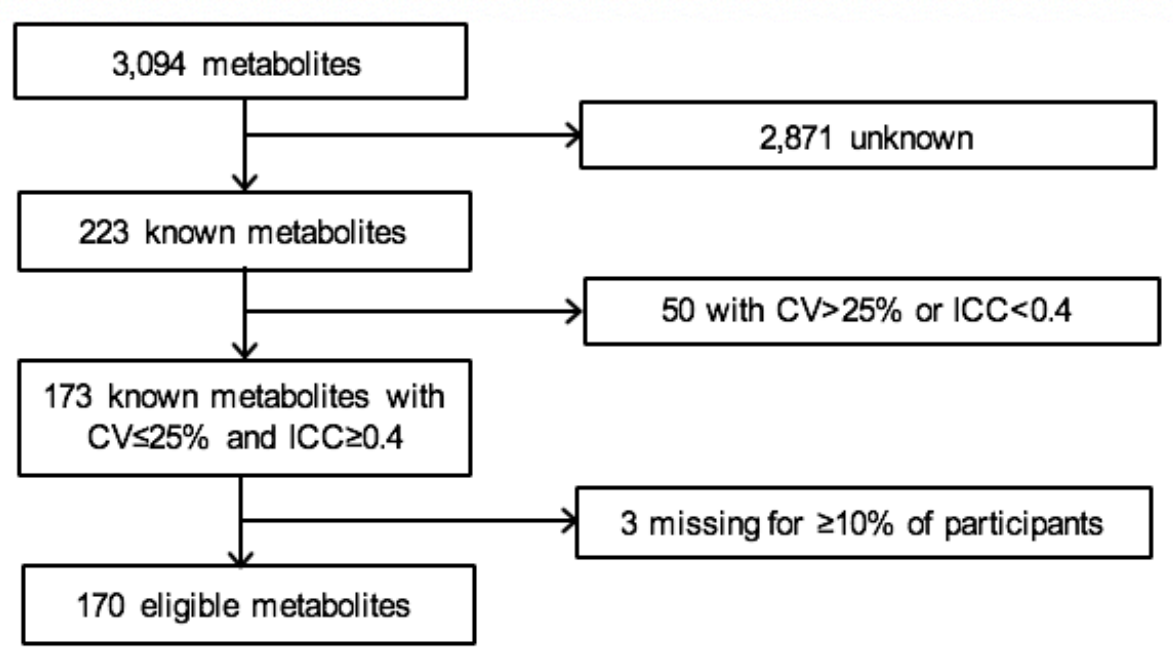




\section{Appendix Figure 2 (Continued)}

\section{PD nested case-control study}

\section{C8-positive method (polar and non-polar lipids)}

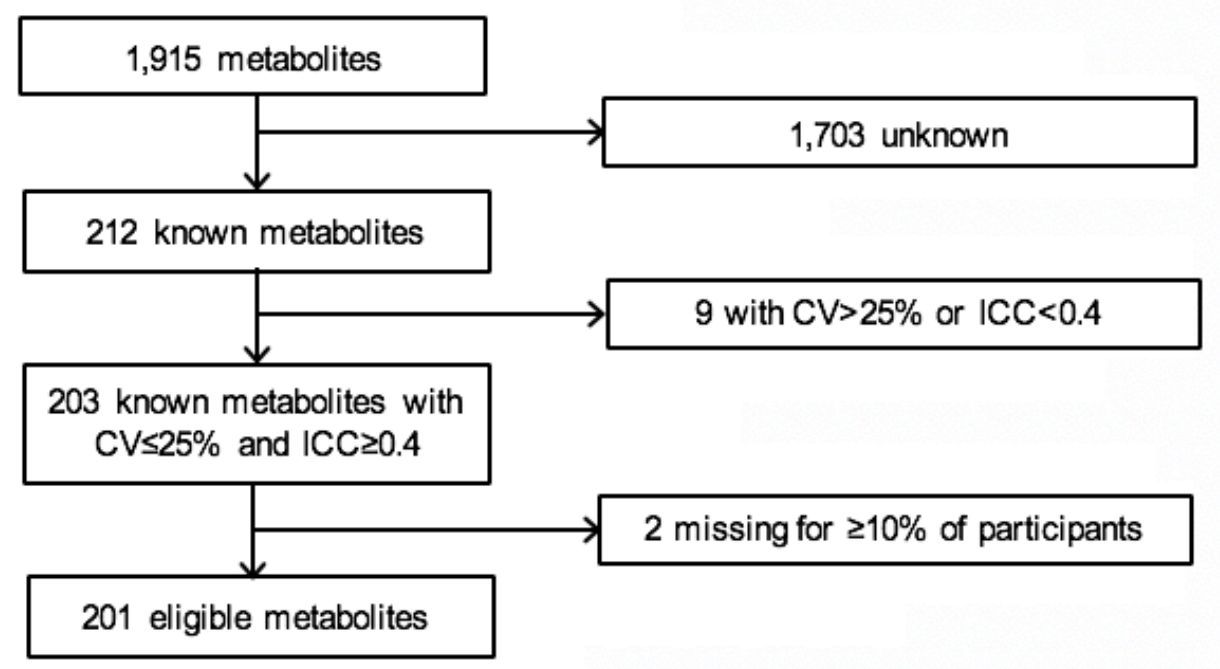

HILIC-positive method (amino acids, dipeptides, and other cationic metabolites)

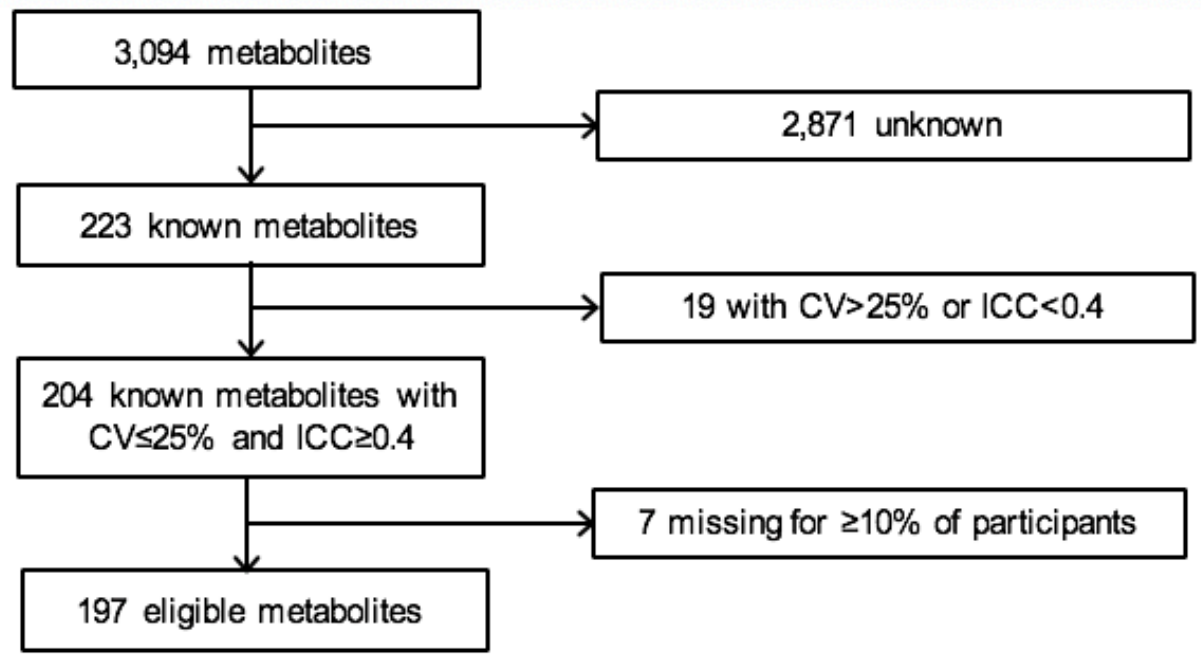




\section{Appendix Figure 2 (Continued)}

\section{Prostate cancer nested case-control study}

C8-positive method (polar and non-polar lipids)

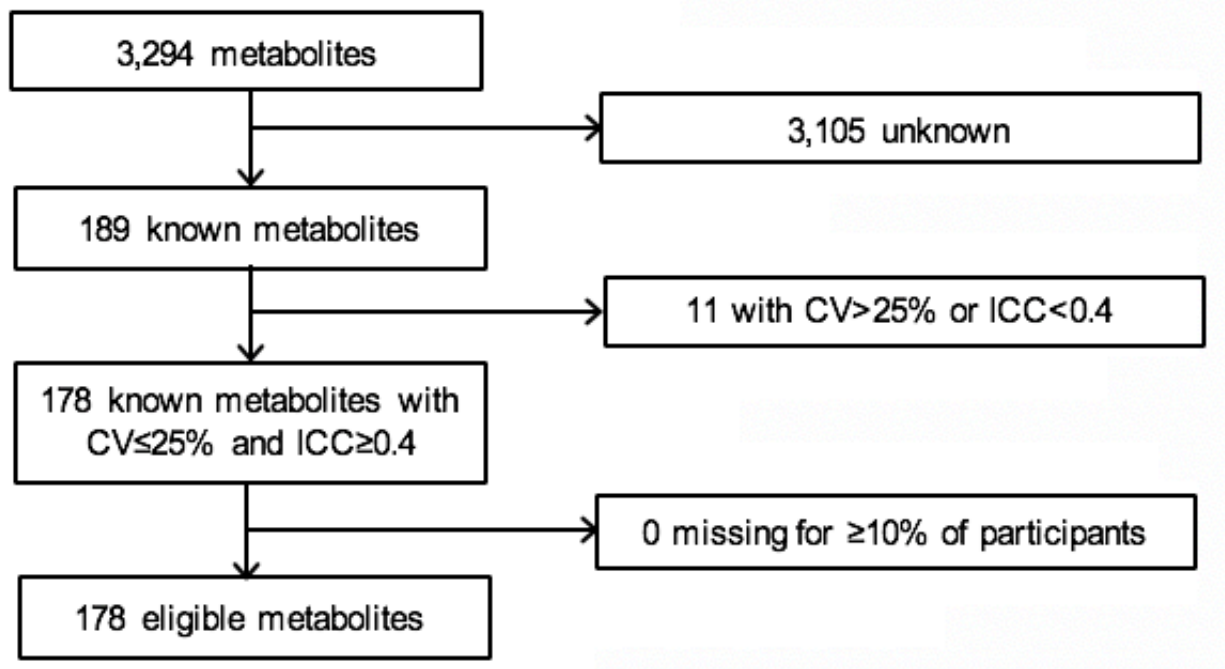

HILIC-positive method (amino acids, dipeptides, and other cationic metabolites)

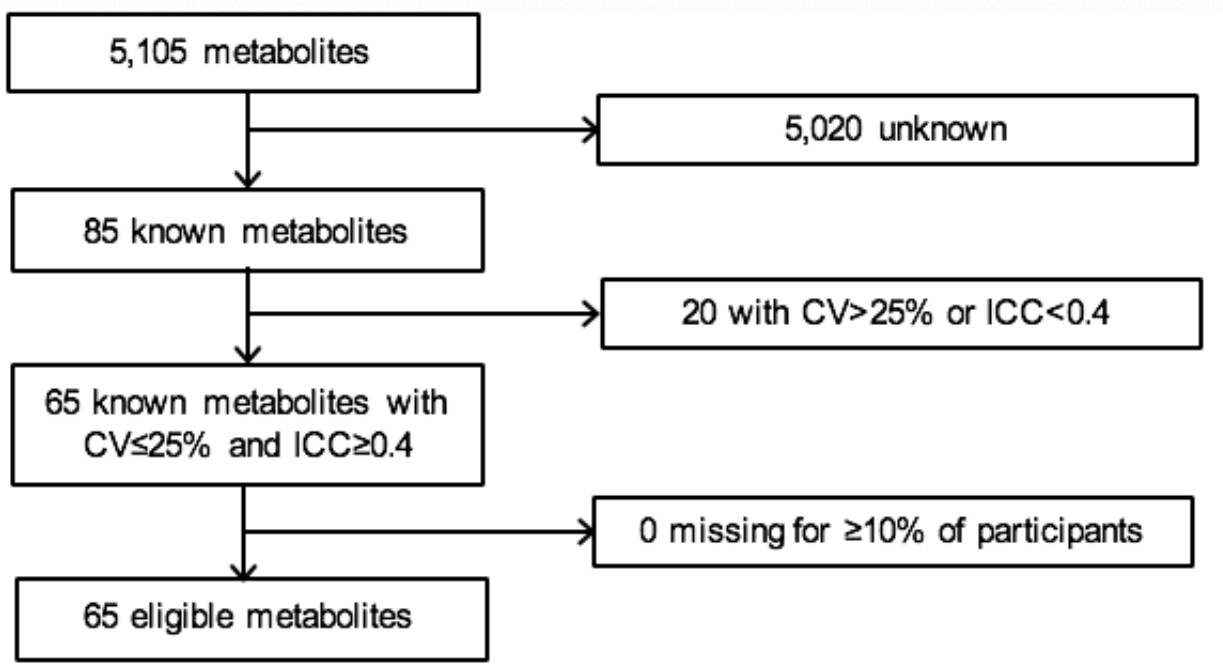


Excluded metabolites before restricting to common metabolites across endpoints

\begin{tabular}{|c|c|}
\hline Basis for exclusion & Metabolites \\
\hline $\begin{array}{l}C V>25 \% \text { or } I C C<0.4 \\
(n=89)\end{array}$ & $\begin{array}{l}\text { C36:4 hydroxy-PC-A } \\
\text { C32:0 PE } \\
\text { 1-methylhistamine } \\
\text { 21-deoxycortisol } \\
\text { 3-(N-acetyl-L-cystein-S-yl) acetaminophen } \\
\text { 3-hydroxyanthranilic acid } \\
\text { 3-methylhistidine } \\
\text { 4-guanidinobutanoic acid } \\
\text { 5-acetylamino-6-formylamino-3-methyluracil } \\
\text { 5-hydroxytryptophan_isomer } \\
\text { acetaminophen glucuronide } \\
\text { acetyl-galactosamine } \\
\text { acetylglycine } \\
\text { adenosine } \\
\text { allantoin } \\
\text { aminoisobutyric acid } \\
\text { anserine } \\
\text { atenolol } \\
\text { beta-alanine } \\
\text { C16:1 LPC } \\
\text { C16:1 MAG } \\
\text { C18:0 MAG } \\
\text { C18:1-OH carnitine } \\
\text { C18:2 SM } \\
\text { C20 carnitine } \\
\text { C22:1 MAG } \\
\text { C22:4 CE } \\
\text { C3-DC-CH3 carnitine } \\
\text { C32:2 DAG } \\
\text { C34:0 PI } \\
\text { C34:1 DAG } \\
\text { C34:2 PC } \\
\text { C34:2 PE plasmalogen } \\
\text { C34:5 PC plasmalogen } \\
\text { C36:0 DAG } \\
\text { C36:3 PS plasmalogen } \\
\text { C38:3 PE plasmalogen } \\
\text { C40:6 PE } \\
\text { C44:13 PE plasmalogen } \\
\text { C47:0 TAG }\end{array}$ \\
\hline
\end{tabular}




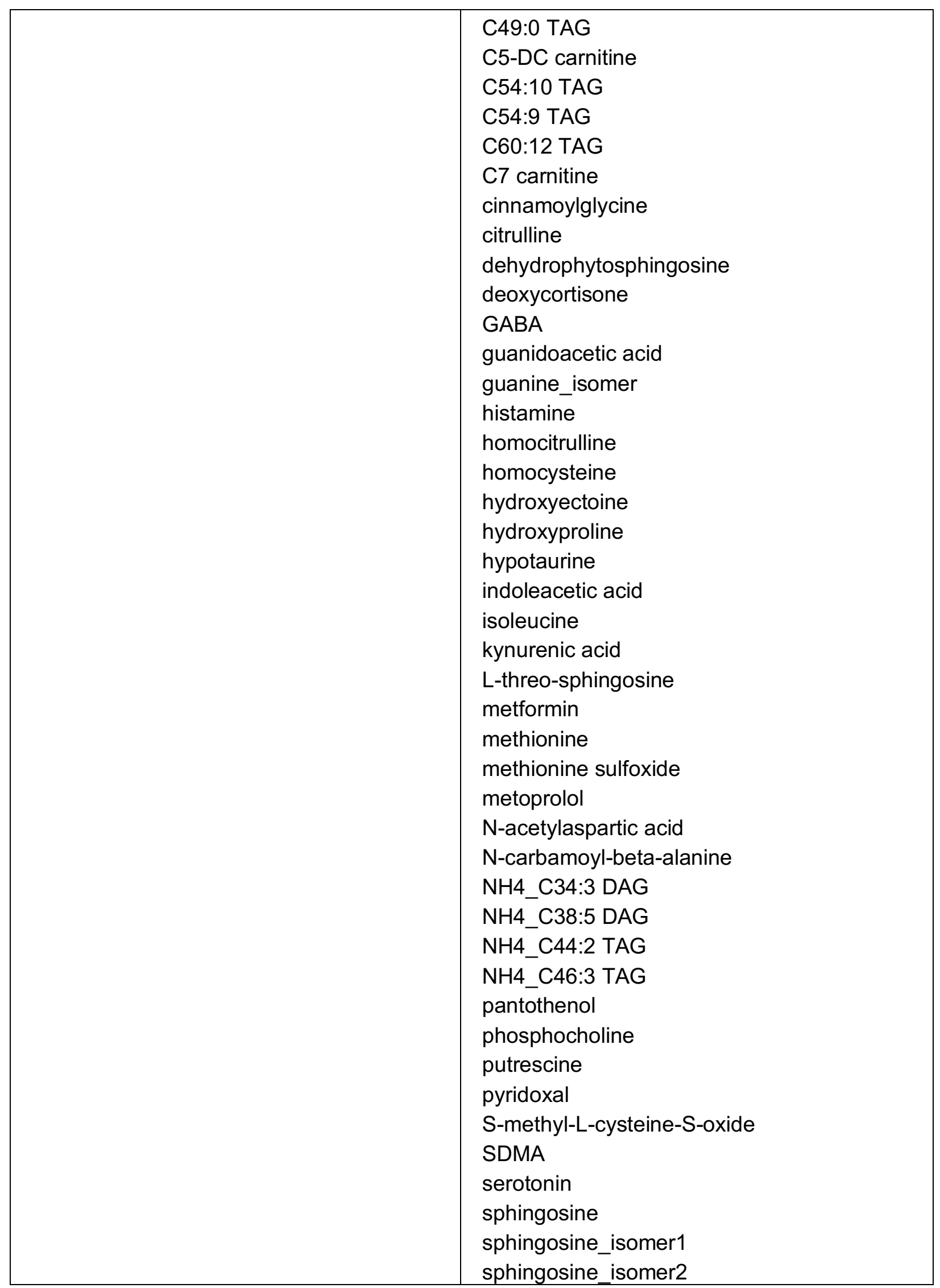




\begin{tabular}{|l|l|}
\hline & sulfamethoxazole \\
& thiamine \\
& urobilinogen \\
& urobilinogen_isomer \\
& urocanic acid \\
& xanthosine \\
\hline Missing for $\geq 10 \%$ of participants & C32:2 DAG \\
$(\mathrm{n}=12)$ & $3-(\mathrm{N}-$-acetyl-L-cystein-S-yl) \\
& acetaminophen \\
& $5-$-hydroxytryptophan_isomer \\
& acetaminophen glucuronide \\
& atenolol \\
& C30:0 DAG \\
& C40:6 PE \\
& cotinine \\
& hydroxycotinine \\
& metoprolol \\
& myristoleic acid \\
& quinine \\
\hline
\end{tabular}


The 198 included metabolites and their categories after restricting to common metabolites across endpoints

\begin{tabular}{|c|c|}
\hline Category & Metabolites \\
\hline Amino acid & $\begin{array}{l}\text { glutamate, glycine, tyrosine, alanine, threonine, asparagine, } \\
\text { histidine, lysine, serine, ornithine, arginine, glutamine, leucine, } \\
\text { valine, tryptophan, phenylalanine }\end{array}$ \\
\hline Carnitine & $\begin{array}{l}\text { C26 carnitine, C5 carnitine, C6 carnitine, C4 carnitine, C16 } \\
\text { carnitine, C18:1 carnitine, C2 carnitine, C4-OH carnitine, } \\
\text { C18:2 carnitine, C12:1 carnitine, carnitine, C8 carnitine, C3 } \\
\text { carnitine, C14 carnitine, C14:2 carnitine, C14:1 carnitine, C10 } \\
\text { carnitine, C10:2 carnitine, C9 carnitine, C5:1 carnitine, C12 } \\
\text { carnitine, C18 carnitine }\end{array}$ \\
\hline Cholesteryl ester (CE) & $\begin{array}{l}\text { C18:2 CE, C16:0 CE, C18:1 CE, C14:0 CE, C20:4 CE, C20:5 } \\
\text { CE, C22:6 CE, C20:3 CE, C18:0 CE, C18:3 CE, C22:5 CE, } \\
\text { C16:1 CE }\end{array}$ \\
\hline Diacylglycerol (DAG) & $\begin{array}{l}\text { C32:0 DAG, C32:1 DAG, C34:1 DAG, C34:2 DAG, C34:3 } \\
\text { DAG, C38:5 DAG, C36:1 DAG, C36:2 DAG, C36:3 DAG, } \\
\text { C36:4 DAG, C38:4 DAG }\end{array}$ \\
\hline $\begin{array}{l}\text { Lysophosphatidylcholine } \\
\text { (LPC) }\end{array}$ & $\begin{array}{l}\text { C18:2 LPC, C20:5 LPC, C18:1 LPC, C22:6 LPC, C20:4 LPC, } \\
\text { C20:3 LPC, C18:0 LPC, C16:0 LPC, C16:1 LPC, C14:0 LPC }\end{array}$ \\
\hline $\begin{array}{l}\text { Lysophosphatidylethanolamine } \\
\text { (LPE) }\end{array}$ & $\begin{array}{l}\text { C16:0 LPE, C18:2 LPE, C18:1 LPE, C18:0 LPE, C22:6 LPE, } \\
\text { C20:4 LPE }\end{array}$ \\
\hline Phosphatidylcholine (PC) & $\begin{array}{l}\text { C38:3 PC, C36:4 PC-A, C40:6 PC, C38:4 PC, C40:10 PC, } \\
\text { C34:2 PC plasmalogen, C34:3 PC plasmalogen, C36:4 PC-B, } \\
\text { C36:5 PC plasmalogen-A, C34:1 PC plasmalogen-A, C36:2 } \\
\text { PC plasmalogen, C36:0 PC, C36:4 PC plasmalogen, C34:4 } \\
\text { PC, C32:2 PC, C36:1 PC plasmalogen, C40:9 PC, C34:0 PC, } \\
\text { C36:3 PC plasmalogen, C38:6 PC, C38:4 PC plasmalogen, } \\
\text { C36:1 PC, C36:3 PC, C34:2 PC, C32:1 PC, C32:0 PC, C30:1 } \\
\text { PC, C36:2 PC, C38:2 PC, C40:7 PC plasmalogen, C36:5 PC } \\
\text { plasmalogen-B, C34:3 PC, C34:1 PC, C38:7 PC plasmalogen, } \\
\text { C30:0 PC }\end{array}$ \\
\hline $\begin{array}{l}\text { Phosphatidylethanolamine } \\
\text { (PE) }\end{array}$ & $\begin{array}{l}\text { C38:2 PE, C38:5 PE, C36:3 PE, C38:6 PE, C36:3 PE } \\
\text { plasmalogen, C40:7 PE plasmalogen, C36:0 PE, C36:4 PE, } \\
\text { C36:4 PE plasmalogen, C36:5 PE plasmalogen, C34:0 PE, } \\
\text { C34:2 PE, C36:2 PE plasmalogen, C34:3 PE plasmalogen, } \\
\text { C36:1 PE, C38:7 PE plasmalogen, C38:4 PE, C38:6 PE } \\
\text { plasmalogen, C38:5 PE plasmalogen, C36:2 PE }\end{array}$ \\
\hline Sphingomyelin (SM) & $\begin{array}{l}\text { C22:0 SM, C20:0 SM, C24:0 SM, C18:2 SM, C22:1 SM, C14:0 } \\
\text { SM, C16:0 SM, C24:1 SM, C18:0 SM }\end{array}$ \\
\hline
\end{tabular}




\begin{tabular}{|c|c|c|}
\hline \multicolumn{2}{|c|}{ Category } & Metabolites \\
\hline \multicolumn{2}{|c|}{ Triacylglycerol (TAG) } & $\begin{array}{l}\text { C52:2 TAG, C50:1 TAG, C52:3 TAG, C50:3 TAG, C50:2 TAG, } \\
\text { C52:1 TAG, C48:2 TAG, C48:3 TAG, C46:1 TAG, C54:2 TAG, } \\
\text { C52:4 TAG, C48:1 TAG, C46:2 TAG, C50:4 TAG, C44:0 TAG, } \\
\text { C54:1 TAG, C48:0 TAG, C50:0 TAG, C46:0 TAG,C54:3 TAG, } \\
\text { C52:0 TAG, C54:5 TAG, C56:5 TAG, C50:5 TAG, C56:3 TAG, } \\
\text { C58:9 TAG, C56:2 TAG, C58:8 TAG, C52:6 TAG, C54:4 TAG, } \\
\text { C54:6 TAG, C50:6 TAG, C58:11 TAG, C58:7 TAG, C56:6 } \\
\text { TAG, C56:10 TAG, C54:7 TAG, C56:9 TAG, C52:7 TAG, } \\
\text { C54:8 TAG, C56:8 TAG, C56:7 TAG }\end{array}$ \\
\hline \multirow[t]{11}{*}{ Others } & $\begin{array}{l}\text { Nitrogenous organic } \\
\text { acid }\end{array}$ & creatine \\
\hline & $\begin{array}{l}\text { Amino acid glycine } \\
\text { derivative }\end{array}$ & dimethylglycine \\
\hline & $\begin{array}{l}\text { Organic compound, } \\
\text { constituent of bile }\end{array}$ & taurine \\
\hline & $\begin{array}{l}\text { Metabolite of } \\
\text { nicotinamide }\end{array}$ & 1-methylnicotinamide \\
\hline & $\begin{array}{l}\text { Carboxylic acid of } \\
\text { piperidine }\end{array}$ & pipecolic acid \\
\hline & TMAO & trimethylamine- $\mathrm{N}$-oxide \\
\hline & Purine nucleoside & 1-methyladenosine \\
\hline & Ceramide & $\begin{array}{l}\text { C16:0 Ceramide (d18:1), C22:0 Ceramide (d18:1), C24:0 } \\
\text { Ceramide (d18:1), C24:1 Ceramide (d18:1) }\end{array}$ \\
\hline & Phosphatidylserine & C40:6 PS, C34:0 PS \\
\hline & $\begin{array}{l}\mathrm{N} \text {-monomethyl-L- } \\
\text { arginine }\end{array}$ & NMMA \\
\hline & $\begin{array}{l}\text { NG,NG-Dimethyl-L- } \\
\text { arginine }\end{array}$ & ADMA \\
\hline
\end{tabular}


Appendix Figure 3 Flowchart of participant selection
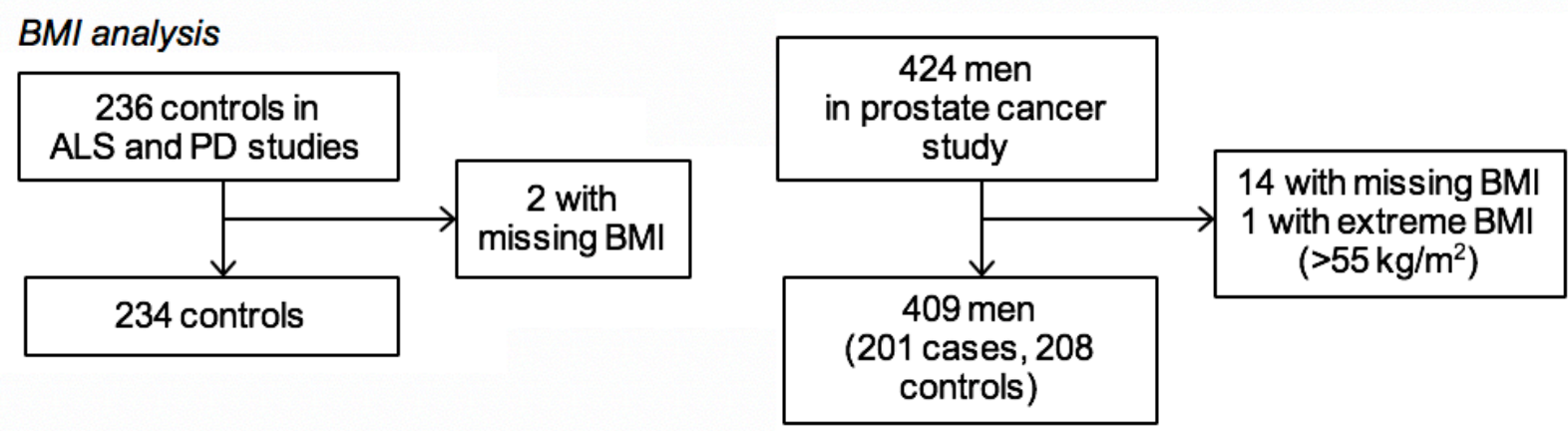

Waist circumference analysis
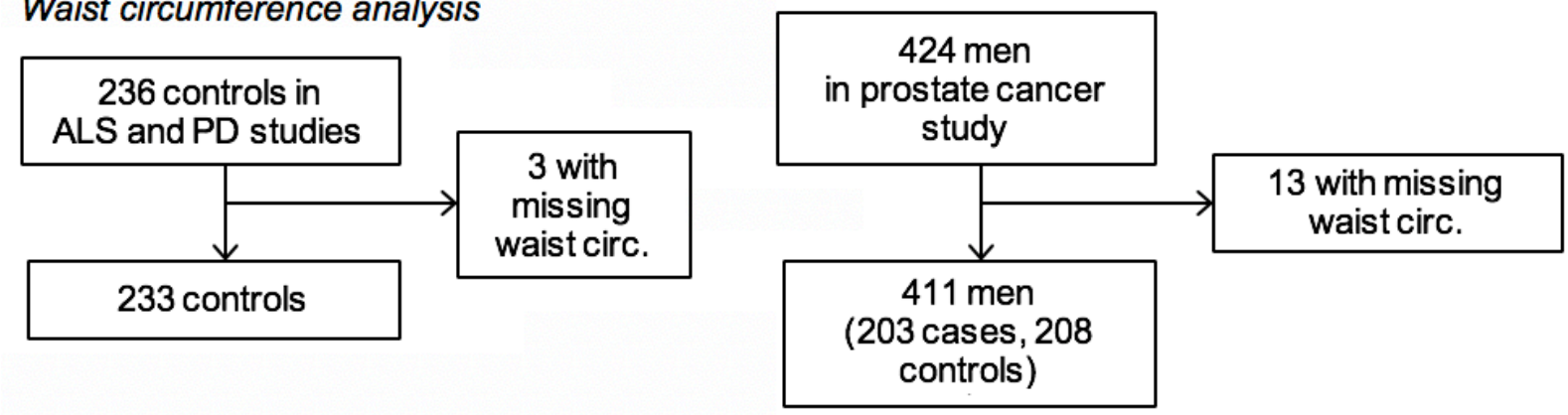

Derived fat mass analysis
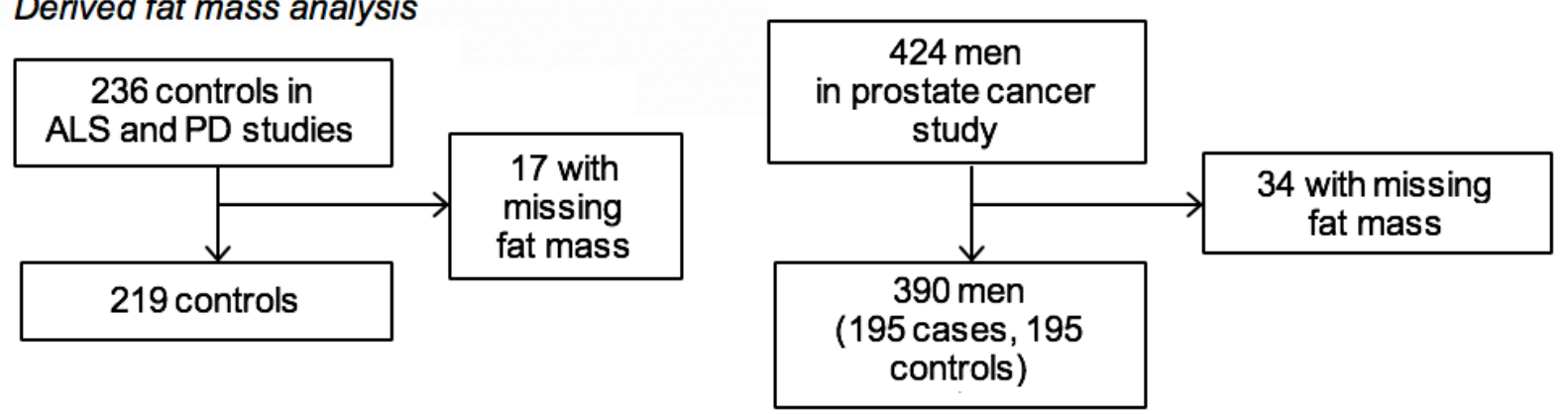


\section{Appendix 3.2}

Appendix Figure 4 Overlap in identified metabolites across adiposity measures (from Table 3.2)

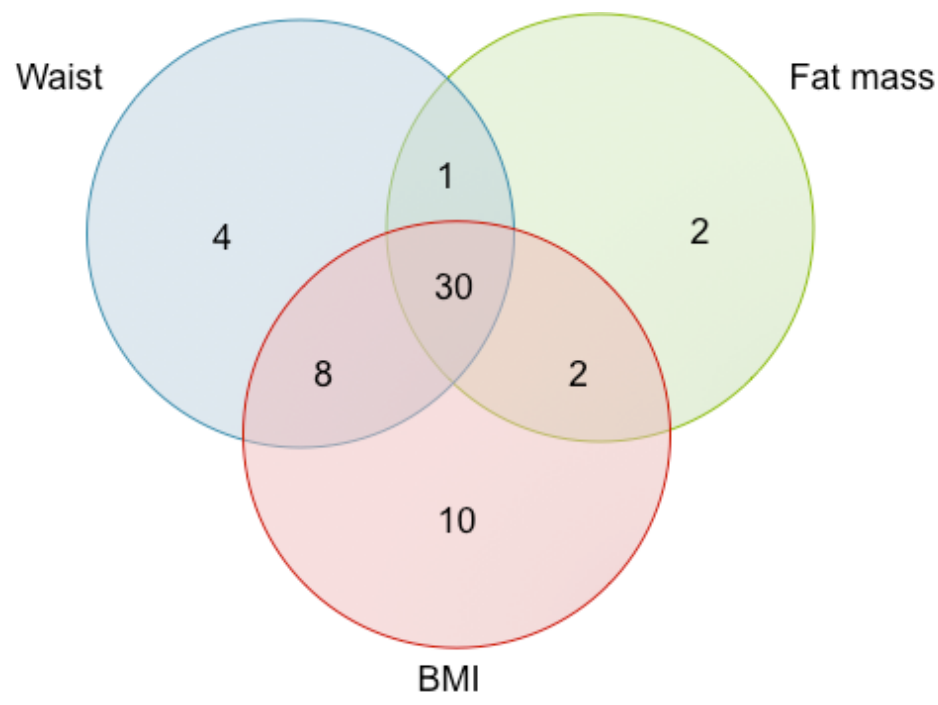

\begin{tabular}{|l|lll|}
\hline Associated with & Metabolite ID & & \\
\hline All three measures $(n=30)$ & HMDB10386 & HMDB10397 & HMDB00148 \\
& HMDB00123 & HMDB11503 & HMDB02815 \\
& HMDB10404 & HMDB07102 & HMDB05369 \\
& HMDB07099 & HMDB05360 & HMDB07098 \\
& HMDB07103 & HMDB05384 & HMDB05433 \\
& HMDB05377 & HMDB07132 & HMDB07218 \\
& HMDB07216 & HMDB10375 & HMDB05367 \\
& HMDB07219 & HMDB05376 & HMDB00641 \\
& HMDB05432 & HMDB10412 & HMDB05403 \\
& HMDB05359 & HMDB00918 & HMDB10419 \\
\hline BMI only ( $=10)$ & & & \\
& HMDB11507 & HMDB07983 & HMDB11130 \\
& HMDB11526 & HMDB08048 & HMDB00688 \\
& HMDB12102 & HMDB07199 & HMDB02013 \\
& HMDB11210 & & \\
\hline Waist circumference only $(n=4)$ & HMDB01348 & HMDB05405 & HMDB05435 \\
& HMDB13326 & & \\
\hline Fat mass only ( $=2)$ & & & \\
\hline BMl and waist circumference only $(n=8)$ & HMDB06733 & HMDB00222 & \\
\hline Waist circumference and fat mass only $(n=1)$ & HMDB12103 & HMDB08047 & HMDB08057 \\
& HMDB06347 & HMDB00705 & HMDB05363 \\
\hline & HMDB07248 & HMDB00610 & \\
\hline & & & \\
\hline
\end{tabular}


Appendix Figure 5 Correlation among metabolites associated with BMI All metabolites $(n=50)$

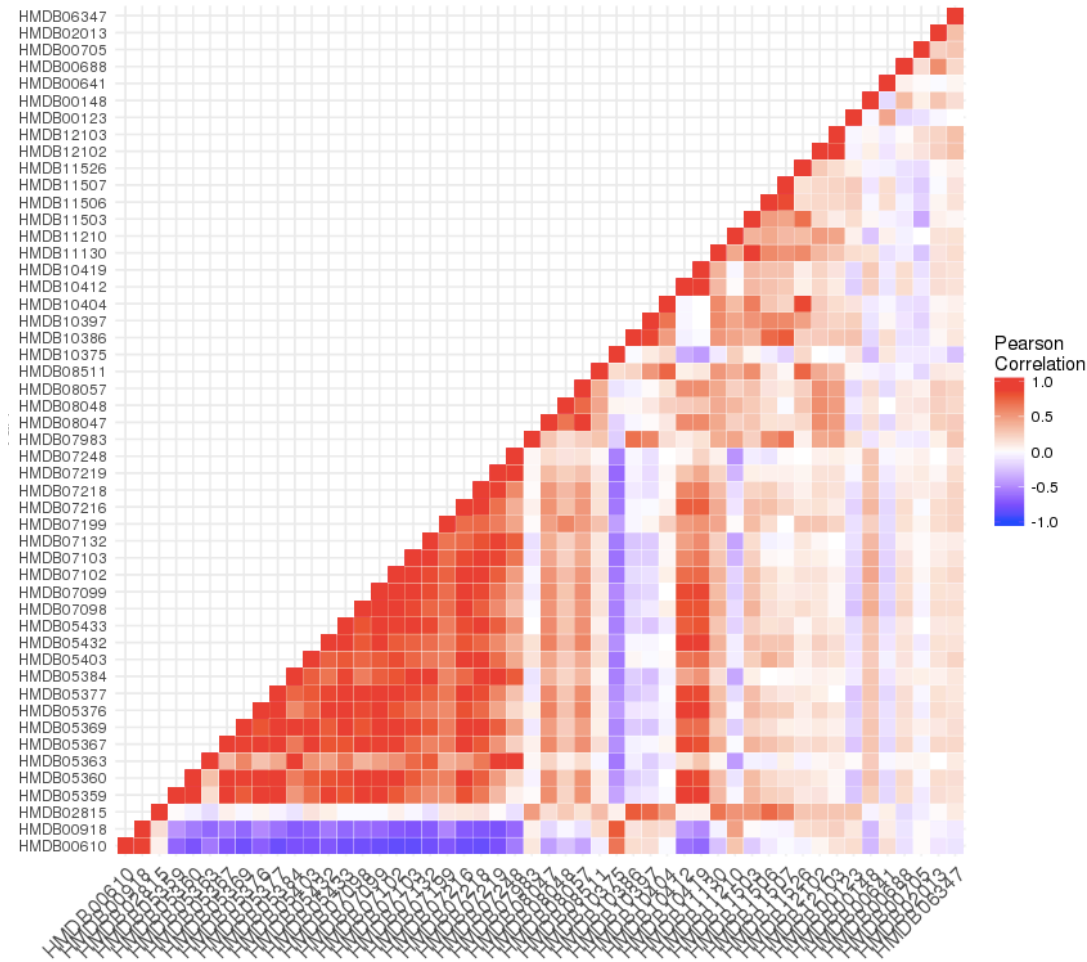

Metabolites with non-zero coefficient in Lasso $(n=15)$

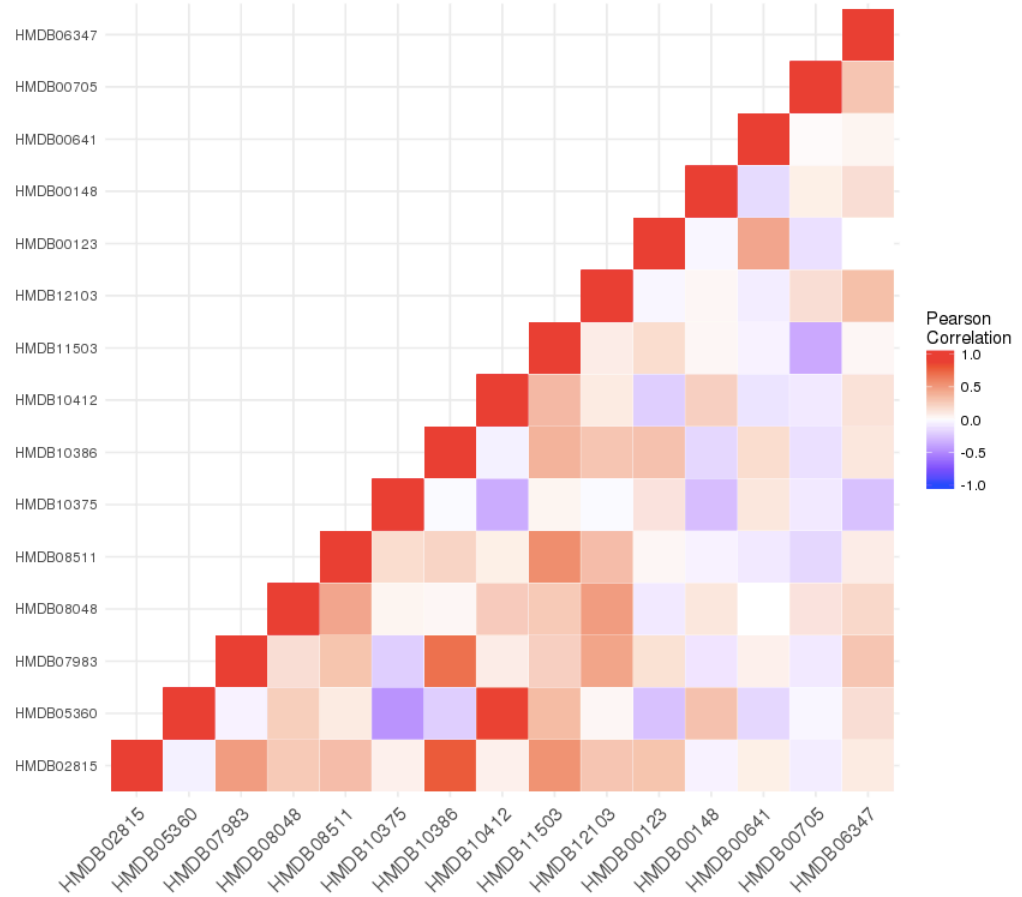


Appendix Figure 6 Correlation among metabolites associated with waist circumference All metabolites ( $n=43)$

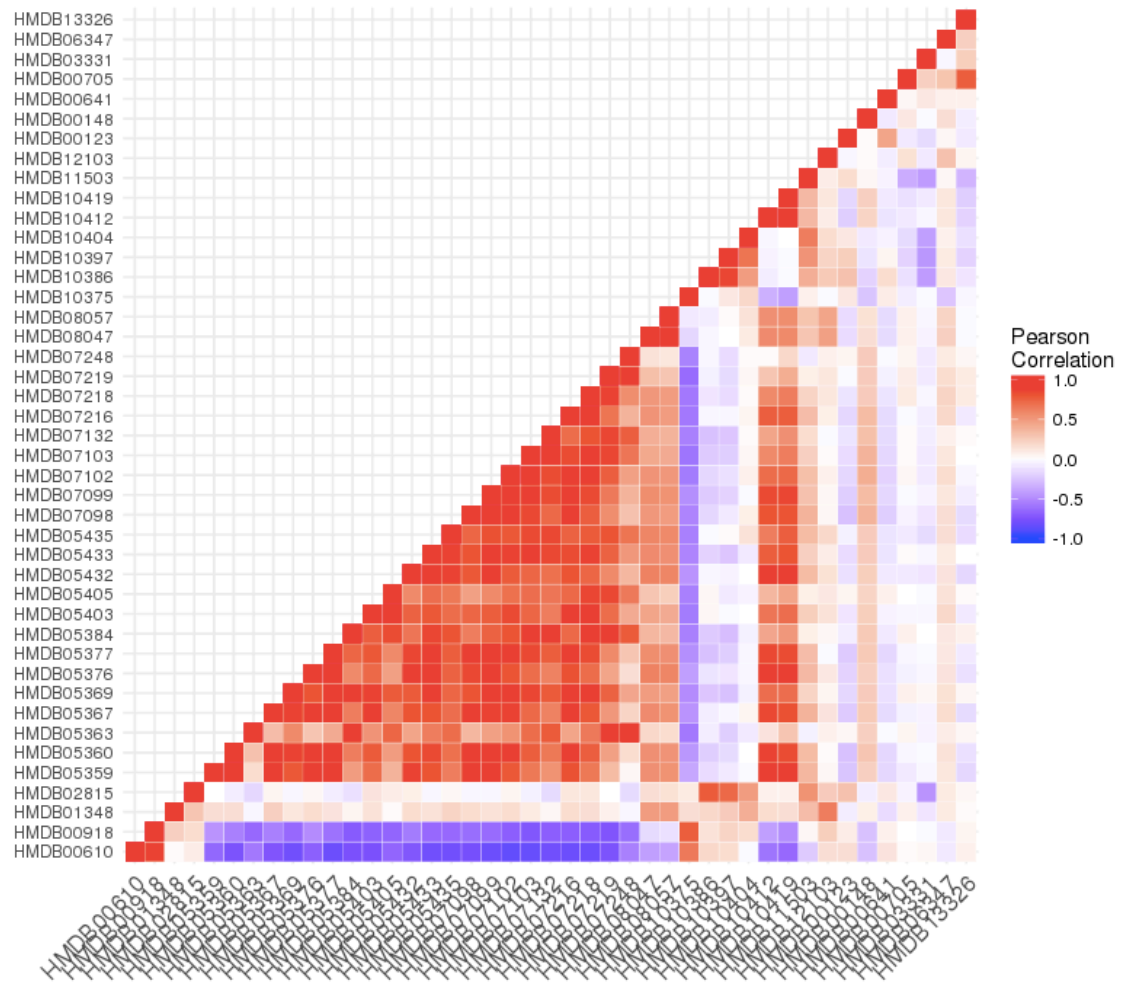

Metabolites with non-zero coefficient in Lasso $(n=15)$

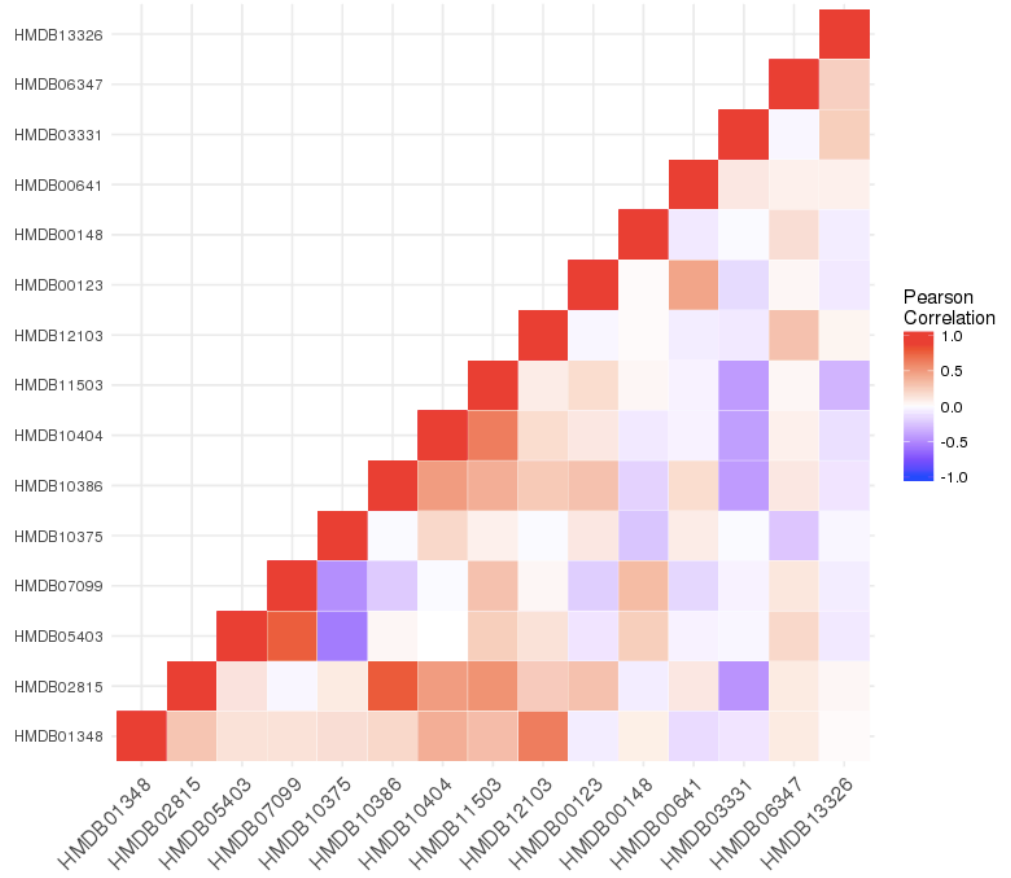


Appendix Figure 7 Correlation among metabolites associated with fat mass

All metabolites $(n=35)$

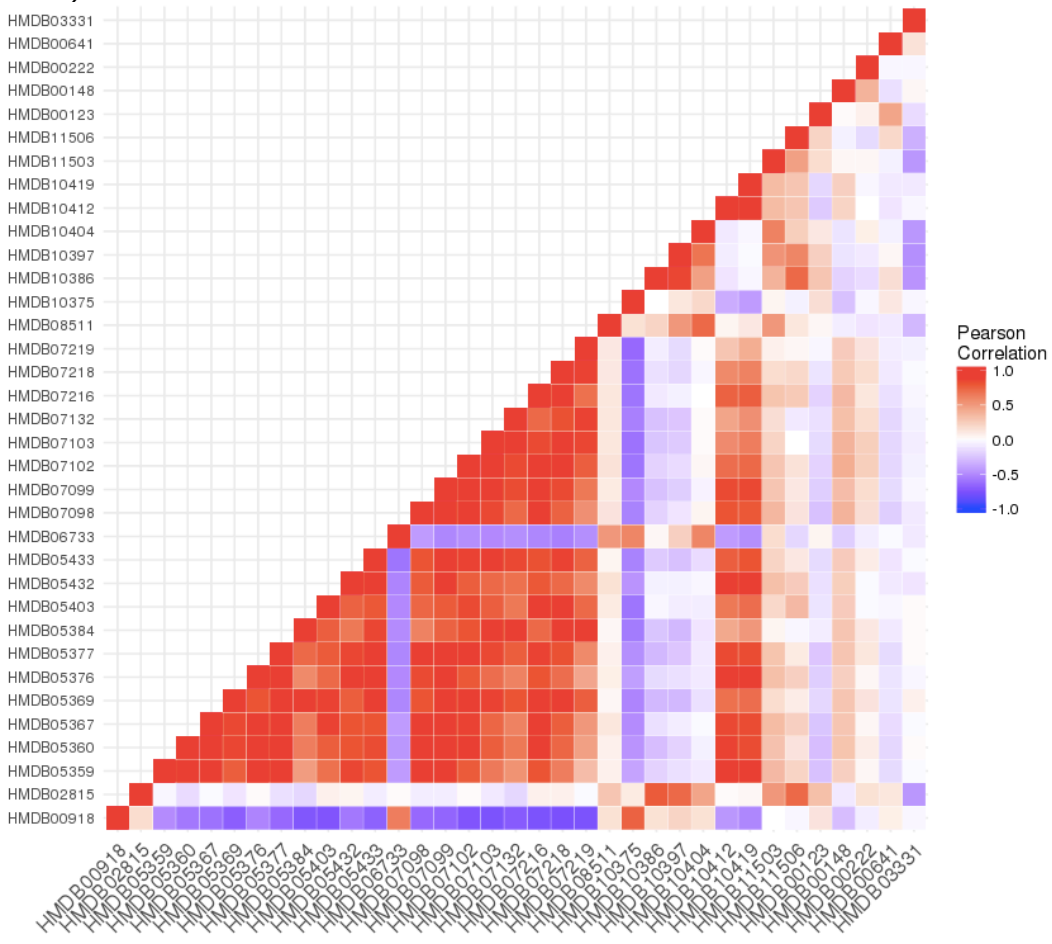

Metabolites with non-zero coefficient in Lasso $(n=27)$

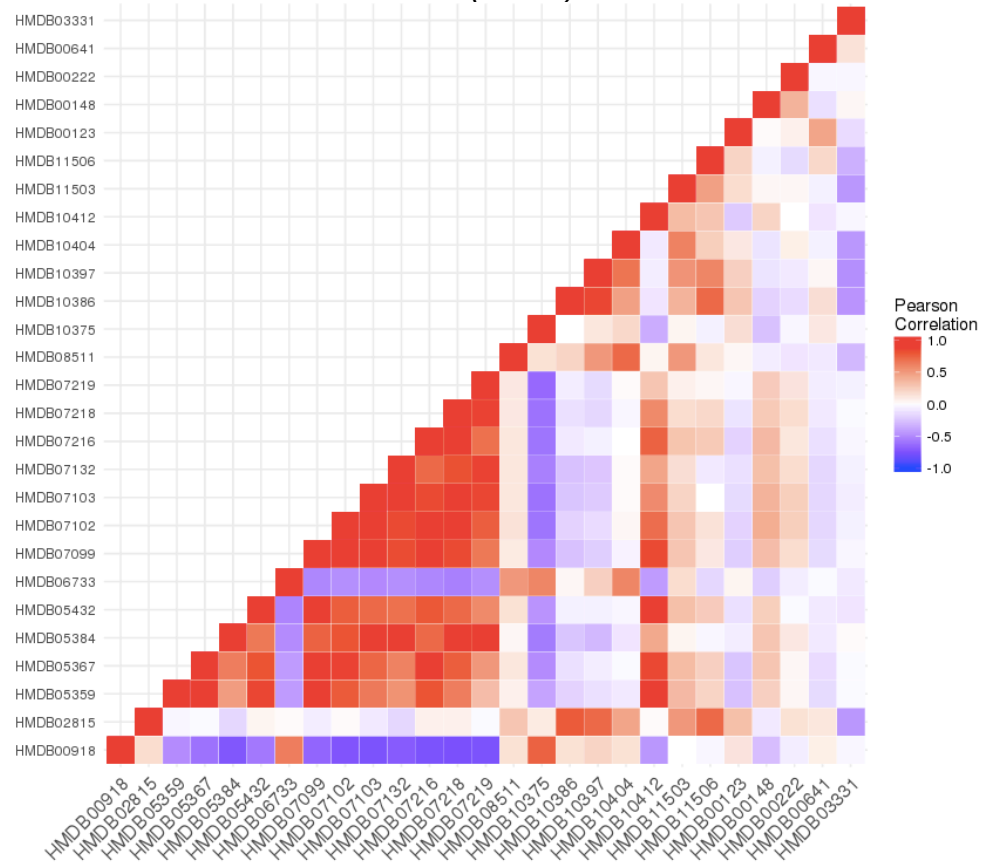


Appendix Table 3 Metabolites associated with adiposity (FDR p-value $<0.05$ and $|r| \geq 0.15$ ) among 217 controls from nested case-control studies with data on all three adiposity measures, Health Professionals Follow-up Study. Italics signify a metabolite identified in Table 3.2 (using all available data on each measure) but not identified in this restricted group.

\begin{tabular}{|c|c|c|c|c|c|c|c|}
\hline \multirow[b]{2}{*}{ HMDB ID } & \multirow[b]{2}{*}{ Metabolite } & \multicolumn{2}{|c|}{ Body mass index } & \multicolumn{2}{|c|}{ Waist circumference } & \multicolumn{2}{|c|}{ Derived fat mass } \\
\hline & & $\begin{array}{r}\text { Pearson } \\
\text { correlation } \\
\text { coefficient }\end{array}$ & $\begin{array}{r}\text { FDR P- } \\
\text { value }\end{array}$ & $\begin{array}{r}\text { Pearson } \\
\text { correlation } \\
\text { coefficient }\end{array}$ & $\begin{array}{l}\text { FDR P. } \\
\text { value }\end{array}$ & $\begin{array}{r}\text { Pearson } \\
\text { correlation } \\
\text { coefficient }\end{array}$ & $\begin{array}{r}\text { FDR P. } \\
\text { value }\end{array}$ \\
\hline HMDB00148 & glutamate & 0.32 & 8.9E-05 & 0.28 & $6.9 \mathrm{E}-03$ & 0.29 & 1.3E-03 \\
\hline HMDB00123 & glycine & -0.30 & 2.3E-04 & -0.21 & 0.04 & -0.25 & $6.1 \mathrm{E}-03$ \\
\hline HMDB00688 & C5 carnitine & 0.18 & 0.04 & -- & -- & -- & -- \\
\hline HMDB06347 & C26 carnitine & 0.18 & 0.04 & -- & -- & -- & -- \\
\hline HMDB00222 & C16 carnitine & -- & -- & -- & -- & 0.18 & 0.04 \\
\hline HMDB00705 & C6 carnitine & & & & & & \\
\hline HMDB02013 & C4 carnitine & & & & & & \\
\hline HMDB10375 & C22:5 CE & -0.20 & 0.02 & -0.22 & 0.03 & -0.24 & 0.01 \\
\hline HMDB00918 & C18:1 CE & -- & -- & -- & -- & -0.19 & 0.04 \\
\hline HMDB06733 & C22:6 CE & -- & -- & -- & -- & -0.19 & 0.04 \\
\hline HMDB00610 & C18:2 CE & & & & & & \\
\hline
\end{tabular}




\begin{tabular}{|c|c|c|c|c|c|c|c|}
\hline \multirow[b]{3}{*}{ HMDB ID } & \multirow[b]{3}{*}{ Metabolite } & $\begin{array}{l}\text { associated wit } \\
\text { all three adip } \\
\text { available data }\end{array}$ & $\begin{array}{l}\text { adiposity ( } \\
\text { ity measu } \\
\text { l each me }\end{array}$ & $\begin{array}{l}R \text { p-value }<0.0 \\
\text { Health Profes } \\
\text { re) but not ider }\end{array}$ & $\begin{array}{l}\text { and }|r| \geq 0 \\
\text { onals Foll } \\
\text { ified in this }\end{array}$ & $\begin{array}{l}\text { ) among } 217 \\
\text { up Study. Itali } \\
\text { stricted group }\end{array}$ & $\begin{array}{l}\text { controls } \\
\text { s signify }\end{array}$ \\
\hline & & \multicolumn{2}{|c|}{ Body mass index } & \multicolumn{2}{|c|}{ Waist circumference } & \multicolumn{2}{|c|}{ Derived fat mass } \\
\hline & & $\begin{array}{c}\text { Pearson } \\
\text { correlation } \\
\text { coefficient }\end{array}$ & $\begin{array}{r}\text { FDR P. } \\
\text { value }\end{array}$ & $\begin{array}{c}\text { Pearson } \\
\text { correlation } \\
\text { coefficient }\end{array}$ & $\begin{array}{c}\text { FDR P. } \\
\text { value }\end{array}$ & $\begin{array}{r}\text { Pearson } \\
\text { correlation } \\
\text { coefficient }\end{array}$ & $\begin{array}{l}\text { FDR P- } \\
\text { value }\end{array}$ \\
\hline \multicolumn{8}{|l|}{$D A G$} \\
\hline \multicolumn{8}{|l|}{ Saturated } \\
\hline HMDB07098 & C32:0 DAG & 0.23 & 0.01 & 0.20 & 0.04 & 0.22 & 0.02 \\
\hline \multicolumn{8}{|l|}{ Unsaturated } \\
\hline HMDB07102 & C34:1 DAG & 0.24 & 7.0E-03 & 0.22 & 0.03 & 0.24 & 0.01 \\
\hline HMDB07099 & C32:1 DAG & 0.23 & 0.01 & 0.22 & 0.03 & 0.24 & 0.01 \\
\hline HMDB07103 & C34:2 DAG & 0.22 & 0.02 & 0.22 & 0.03 & 0.23 & 0.01 \\
\hline HMDB07216 & C36:1 DAG & 0.21 & 0.02 & 0.21 & 0.04 & 0.22 & 0.02 \\
\hline HMDB07218 & C36:2 DAG & 0.21 & 0.02 & 0.20 & 0.04 & 0.21 & 0.02 \\
\hline HMDB07132 & C34:3 DAG & 0.21 & 0.02 & 0.22 & 0.03 & 0.23 & 0.01 \\
\hline HMDB07219 & C36:3 DAG & 0.19 & 0.03 & 0.19 & 0.04 & 0.20 & 0.03 \\
\hline HMDB07248 & C36:4 DAG & & & & & & \\
\hline HMDB07199 & C38:5 DAG & & & & & & \\
\hline \multicolumn{8}{|l|}{$L P C$} \\
\hline HMDB10386 & C18:2 LPC & -0.36 & 8.4E-06 & -0.24 & 0.02 & -0.30 & 1.1E-03 \\
\hline HMDB10397 & C20:5 LPC & -0.35 & 1.3E-05 & -0.24 & 0.02 & -0.29 & $1.1 \mathrm{E}-03$ \\
\hline HMDB02815 & C18:1 LPC & -0.31 & 2.3E-04 & -0.23 & 0.03 & -0.27 & $3.8 \mathrm{E}-03$ \\
\hline HMDB10404 & C22:6 LPC & -0.27 & $1.4 \mathrm{E}-03$ & -0.21 & 0.03 & -0.26 & 5.5E-03 \\
\hline
\end{tabular}


Appendix Table 3 (Continued) Metabolites associated with adiposity (FDR p-value $<0.05$ and $|r| \geq 0.15$ ) among 217 controls from nested case-control studies with data on all three adiposity measures, Health Professionals Follow-up Study. Italics signify a metabolite identified in Table 3.2 (using all available data on each measure) but not identified in this restricted group.

\begin{tabular}{|c|c|c|c|c|c|c|c|}
\hline \multirow[b]{2}{*}{ HMDB ID } & \multirow[b]{2}{*}{ Metabolite } & \multicolumn{2}{|c|}{ Body mass index } & \multicolumn{2}{|c|}{ Waist circumference } & \multicolumn{2}{|c|}{ Derived fat mass } \\
\hline & & $\begin{array}{c}\text { Pearson } \\
\text { correlation } \\
\text { coefficient }\end{array}$ & $\begin{array}{l}\text { FDR P. } \\
\text { value }\end{array}$ & $\begin{array}{c}\text { Pearson } \\
\text { correlation } \\
\text { coefficient }\end{array}$ & $\begin{array}{l}\text { FDR P. } \\
\text { value }\end{array}$ & $\begin{array}{c}\text { Pearson } \\
\text { correlation } \\
\text { coefficient }\end{array}$ & $\begin{array}{l}\text { FDR P- } \\
\text { value }\end{array}$ \\
\hline \multicolumn{8}{|l|}{ LPE } \\
\hline HMDB11503 & C16:0 LPE & -0.29 & $4.2 \mathrm{E}-04$ & -0.20 & 0.04 & -0.23 & 0.01 \\
\hline HMDB11507 & C18:2 LPE & -0.29 & 4.4E-04 & -- & -- & -0.18 & 0.04 \\
\hline HMDB11506 & C18:1 LPE & -0.24 & $8.9 \mathrm{E}-03$ & -- & -- & -0.19 & 0.04 \\
\hline HMDB11130 & C18:0 LPE & -0.21 & 0.02 & -- & -- & -- & -- \\
\hline HMDB11526 & C22:6 LPE & -0.20 & 0.02 & -- & -- & -- & -- \\
\hline \multicolumn{8}{|l|}{$P C$} \\
\hline HMDB08047 & C38:3 PC & -0.29 & $4.2 \mathrm{E}-04$ & -- & -- & -- & -- \\
\hline HMDB07983 & C36:4 PC-A & -0.29 & 4.4E-04 & -- & -- & -- & -- \\
\hline HMDB08048 & C38:4 PC & -0.24 & 8.9E-03 & -- & -- & -- & -- \\
\hline HMDB08057 & $\mathrm{C} 40: 6 \mathrm{PC}$ & -0.21 & 0.02 & -- & -- & -- & -- \\
\hline HMDB08511 & C40:10 PC & -- & -- & -- & -- & -0.18 & 0.04 \\
\hline HMDB11210 & C34:2 PC plasmalogen & & & & & & \\
\hline \multicolumn{8}{|l|}{$S M$} \\
\hline HMDB12103 & C22:0 SM & 0.22 & 0.02 & -- & -- & -- & -- \\
\hline HMDB01348 & C18:0 SM & -- & -- & 0.19 & 0.04 & -- & -- \\
\hline HMDB12102 & C20:0 SM & & & & & & \\
\hline
\end{tabular}


Appendix Table 3 (Continued) Metabolites associated with adiposity (FDR p-value $<0.05$ and $|r| \geq 0.15$ ) among 217 controls from nested case-control studies with data on all three adiposity measures, Health Professionals Follow-up Study. Italics signify a metabolite identified in Table 3.2 (using all available data on each measure) but not identified in this restricted group.

\begin{tabular}{|c|c|c|c|c|c|c|c|}
\hline \multirow[b]{2}{*}{ HMDB ID } & \multirow[b]{2}{*}{ Metabolite } & \multicolumn{2}{|c|}{ Body mass index } & \multicolumn{2}{|c|}{ Waist circumference } & \multicolumn{2}{|c|}{ Derived fat mass } \\
\hline & & $\begin{array}{r}\text { Pearson } \\
\text { correlation } \\
\text { coefficient }\end{array}$ & $\begin{array}{r}\text { FDR P- } \\
\text { value }\end{array}$ & $\begin{array}{c}\text { Pearson } \\
\text { correlation } \\
\text { coefficient }\end{array}$ & $\begin{array}{l}\text { FDR P- } \\
\text { value }\end{array}$ & $\begin{array}{r}\text { Pearson } \\
\text { correlation } \\
\text { coefficient }\end{array}$ & $\begin{array}{r}\text { FDR P- } \\
\text { value }\end{array}$ \\
\hline \multicolumn{8}{|l|}{ TAG } \\
\hline \multicolumn{8}{|l|}{ Unsaturated } \\
\hline HMDB05369 & C52:2 TAG & 0.24 & 7.0E-03 & 0.21 & 0.04 & 0.22 & 0.01 \\
\hline HMDB05360 & C50:1 TAG & 0.23 & $9.6 \mathrm{E}-03$ & 0.19 & 0.04 & 0.22 & 0.02 \\
\hline HMDB05384 & C52:3 TAG & 0.22 & 0.02 & 0.20 & 0.04 & 0.21 & 0.02 \\
\hline HMDB05377 & C50:2 TAG & 0.22 & 0.02 & 0.19 & 0.04 & 0.21 & 0.02 \\
\hline HMDB05433 & C50:3 TAG & 0.21 & 0.02 & 0.20 & 0.04 & 0.22 & 0.02 \\
\hline HMDB05367 & C52:1 TAG & 0.21 & 0.02 & 0.19 & 0.05 & 0.21 & 0.02 \\
\hline HMDB05376 & C48:2 TAG & 0.19 & 0.03 & -- & -- & 0.20 & 0.03 \\
\hline HMDB05403 & C54:2 TAG & 0.18 & 0.04 & -- & -- & 0.19 & 0.03 \\
\hline HMDB10412 & C46:1 TAG & 0.18 & 0.05 & -- & -- & 0.19 & 0.04 \\
\hline HMDB05432 & C48:3 TAG & -- & -- & -- & -- & 0.19 & 0.03 \\
\hline HMDB05363 & C52:4 TAG & -- & -- & -- & -- & 0.18 & 0.05 \\
\hline HMDB10419 & C46:2 TAG & -- & -- & -- & -- & 0.18 & 0.05 \\
\hline HMDB05359 & C48:1 TAG & & & & & & \\
\hline HMDB05405 & C54:3 TAG & & & & & & \\
\hline HMDB05435 & C50:4 TAG & & & & & & \\
\hline
\end{tabular}


Appendix Table 3 (Continued) Metabolites associated with adiposity (FDR p-value $<0.05$ and $|r| \geq 0.15$ ) among 217 controls from nested case-control studies with data on all three adiposity measures, Health Professionals Follow-up Study. Italics signify a metabolite identified in Table 3.2 (using all available data on each measure) but not identified in this restricted group.

\begin{tabular}{|c|c|c|c|c|c|c|c|}
\hline \multirow[b]{2}{*}{ HMDB ID } & \multirow[b]{2}{*}{ Metabolite } & \multicolumn{2}{|c|}{ Body mass index } & \multicolumn{2}{|c|}{ Waist circumference } & \multicolumn{2}{|c|}{ Derived fat mass } \\
\hline & & $\begin{array}{r}\text { Pearson } \\
\text { correlation } \\
\text { coefficient }\end{array}$ & $\begin{array}{l}\text { FDR P. } \\
\text { value }\end{array}$ & $\begin{array}{r}\text { Pearson } \\
\text { correlation } \\
\text { coefficient }\end{array}$ & $\begin{array}{l}\text { FDR P- } \\
\text { value }\end{array}$ & $\begin{array}{c}\text { Pearson } \\
\text { correlation } \\
\text { coefficient }\end{array}$ & $\begin{array}{r}\text { FDR P- } \\
\text { value }\end{array}$ \\
\hline \multicolumn{8}{|c|}{$\begin{array}{l}\text { Purines, } \\
\text { pyrimidines, } \\
\text { and derivatives }\end{array}$} \\
\hline HMDB03331 & 1-methyladenosine & -- & -- & -- & -- & 0.19 & 0.04 \\
\hline
\end{tabular}

CE, cholesterol ester; DAG, diacylglycerol; LPC, lysophosphatidylcholine; LPE, lysophosphatidylethanolamine; PC, phosphatidylcholine; PE, phosphatidylethanolamine; SM, sphingomyeline; TAG, triacylglycerol.

Estimates from partial Pearson correlation, adjusted for age at blood draw (continuous) and smoking status (ever/never). 37 metabolites associated with BMI, 23 with waist circumference, 36 with derived fat mass. 
Appendix 3.3 Lasso models used for predicting adiposity using associated metabolites

Model $1 \mathrm{BMI}$ model

Dependent variable: BMI $\left(\mathrm{kg} / \mathrm{m}^{2}\right)$

\begin{tabular}{|c|c|}
\hline (Intercept) & 25.481967043 \\
\hline Z_LN_HMDB00610 & \\
\hline Z_LN_HMDB00918 & \\
\hline Z_LN_HMDB02815 & -0.227082736 \\
\hline Z_LN_HMDB05359 & . \\
\hline Z_LN_HMDB05360 & 0.247409944 \\
\hline Z_LN_HMDB05363 & · \\
\hline Z_LN_HMDB05367 & . \\
\hline Z_LN_HMDB05369 & . \\
\hline Z_LN_HMDB05376 & . \\
\hline Z_LN_HMDB05377 & . \\
\hline Z_LN_HMDB05384 & . \\
\hline Z_LN_HMDB05403 & . \\
\hline Z_LN_HMDB05432 & . \\
\hline Z_LN_HMDB05433 & . \\
\hline Z_LN_HMDB07098 & . \\
\hline Z_LN_HMDB07099 & . \\
\hline Z_LN_HMDB07102 & . \\
\hline Z_LN_HMDB07103 & . \\
\hline Z_LN_HMDB07132 & . \\
\hline Z_LN_HMDB07199 & . \\
\hline Z_LN_HMDB07216 & . \\
\hline Z_LN_HMDB07218 & . \\
\hline Z_LN_HMDB07219 & . \\
\hline Z_LN_HMDB07248 & \\
\hline Z_LN_HMDB07983 & -0.469240982 \\
\hline Z_LN_HMDB08047 & · \\
\hline Z_LN_HMDB08048 & 0.323098308 \\
\hline Z_LN_HMDB08057 & 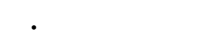 \\
\hline Z_LN_HMDB08511 & -0.133746720 \\
\hline Z_LN_HMDB10375 & -0.156273616 \\
\hline Z_LN_HMDB10386 & -0.002009422 \\
\hline Z_LN_HMDB10397 & . \\
\hline Z_LN_HMDB10404 & . \\
\hline Z_LN_HMDB10412 & 0.101500366 \\
\hline Z_LN_HMDB10419 & 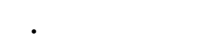 \\
\hline Z_LN_HMDB11130 & . \\
\hline Z_LN_HMDB11210 & . \\
\hline Z_LN_HMDB11503 & -0.634275737 \\
\hline Z_LN_HMDB11506 & · \\
\hline Z_LN_HMDB11507 & . \\
\hline Z_LN_HMDB11526 & . \\
\hline Z_LN_HMDB12102 & · \\
\hline Z_LN_HMDB12103 & 0.656748333 \\
\hline Z_LN_HMDB00123 & -0.235639131 \\
\hline Z_LN_HMDB00148 & 0.433652273 \\
\hline Z_LN_HMDB00641 & -0.211363071 \\
\hline Z_LN_HMDB00688 & \\
\hline Z_LN_HMDB00705 & -0.031189221 \\
\hline Z_LN_HMDB02013 & \\
\hline Z_LN_HMDB06347 & 0.207153864 \\
\hline
\end{tabular}


Model 2 Waist circumference model

Dependent variable: Waist circumference $(\mathrm{cm})$

$\begin{array}{ll}\text { (Intercept) } & 95.16245283 \\ \text { Z_LN_HMDB00610 } & \cdot \\ \text { Z_LN_HMDB00918 } & \cdot \\ \text { Z_LN_HMDB01348 } & 1.17826711 \\ \text { Z_LN_HMDB02815 } & -0.46634006 \\ \text { Z_LN_HMDB05359 } & \cdot \\ \text { Z_LN_HMDB05360 } & \cdot \\ \text { Z_LN_HMDB05363 } & \cdot \\ \text { Z_LN_HMDB05367 } & \cdot \\ \text { Z_LN_HMDB05369 } & \cdot \\ \text { Z_LN_HMDB05376 } & \cdot \\ \text { Z_LN_HMDB05377 } & \cdot \\ \text { Z_LN_HMDB05384 } & \cdot \\ \text { Z_LN_HMDB05403 } & \cdot \\ \text { Z_LN_HMDB05405 } & \cdot \\ \text { Z_LN_HMDB05432 } & \cdot \\ \text { Z_LN_HMDB05433 } & \cdot \\ \text { Z_LN_HMDB05435 } & \cdot \\ \text { Z_LN_HMDB07098 } & \cdot \\ \text { Z_LN_HMDB07099 } & \cdot \\ \text { Z_LN_HMDB07102 } & \cdot \\ \text { Z_LN_HMDB07103 } & \cdot \\ \text { Z_LN_HMDB07132 } & \cdot \\ \text { Z_LN_HMDB07216 } & \cdot \\ \text { Z_LN_HMDB07218 } & \cdot \\ \text { Z_LN_HMDB07219 } & \cdot \\ \text { Z_LN_HMDB07248 } & \cdot \\ \text { Z_LN_HMDB08047 } & \cdot \\ \text { Z_LN_HMDB08057 } & \cdot \\ \text { Z_LN_HMDB10375 } & -0.52688780 \\ \text { Z_LN_HMDB10386 } & -0.53632774 \\ \text { Z_LN_HMDB10397 } & \cdot \\ \text { Z_LN_HMDB10404 } & -0.65210727 \\ \text { Z_LN_HMDB10412 } & \cdot \\ \text { Z_LN_HMDB10419 } & \cdot \\ \text { Z_LN_HMDB11503 } & -0.59170501 \\ \text { Z_LN_HMDB12103 } & 0.52483890 \\ \text { Z_LN_HMDB00123 } & -0.08047205 \\ \text { Z_LN_HMDB00148 } & 1.24732308 \\ \text { Z_LN_HMDB00641 } & -0.75792644 \\ \text { Z_LN_HMDB00705 } & \cdot \\ \text { Z_LN_HMDB03331 } & \cdot \\ \text { Z_LN_HMDB06347 } & 0.68950492 \\ \text { Z_LN_HMDB13326 } & 0.68972881 \\ & \end{array}$


Model 3 Fat mass model

Dependent variable: Fat mass $(\mathrm{kg})$

(Intercept) $\quad 21.7543242619$

Z_LN_HMDB00918 1.3191195236

Z_LN_HMDB02815 - 0.7637223346

Z_LN_HMDB05359 -2.2962391665

Z_LN_HMDB05360 .

Z_LN_HMDB05367 1.5349426089

Z_LN_HMDB05369.

Z_LN_HMDB05376 .

Z_LN_HMDB05377.

Z_LN_HMDB05384 -1.4524478925

Z_LN_HMDB05403 .

Z_LN_HMDB05432 1.4548015023

Z_LN_HMDB05433 .

Z_LN_HMDB06733 0.5235328093

Z_LN_HMDB07098.

Z_LN_HMDB07099 0.3795070343

Z_LN_HMDB07102 1.7596482670

Z_LN_HMDB07103 -2.2874496147

Z_LN_HMDB07132 0.4765560277

Z_LN_HMDB07216 0.0086633264

Z_LN_HMDB07218 0.1171935501

Z_LN_HMDB07219 1.6581611560

Z_LN_HMDB08511 -0.7844008215

Z_LN_HMDB10375 - 0.8321392146

Z_LN_HMDB10386 -0.4601701951

Z_LN_HMDB10397 0.1791226877

Z_LN_HMDB10404 - 0.3754155929

Z_LN_HMDB10412 0.7364368518

Z_LN_HMDB10419 .

Z_LN_HMDB11503 -0.6677177773

Z_LN_HMDB11506 - 0.0003869818

Z_LN_HMDB00123 - 0.4536297224

Z_LN_HMDB00148 0.5299459534

Z_LN_HMDB00222 0.8788935590

Z_LN_HMDB00641 -0.4963226144

Z_LN_HMDB03331 0.1766192939 
Appendix 3.4 Results from additional analyses

A

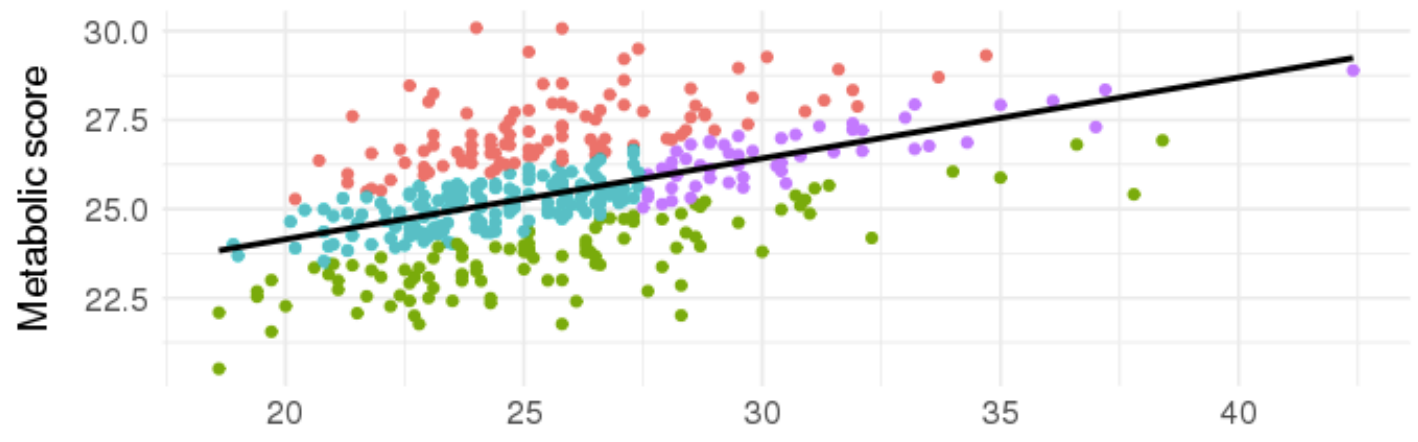

BMI $\left(\mathrm{kg} / \mathrm{m}^{2}\right)$

B Odds ratios for advanced prostate cancer by BMI-metabolic group

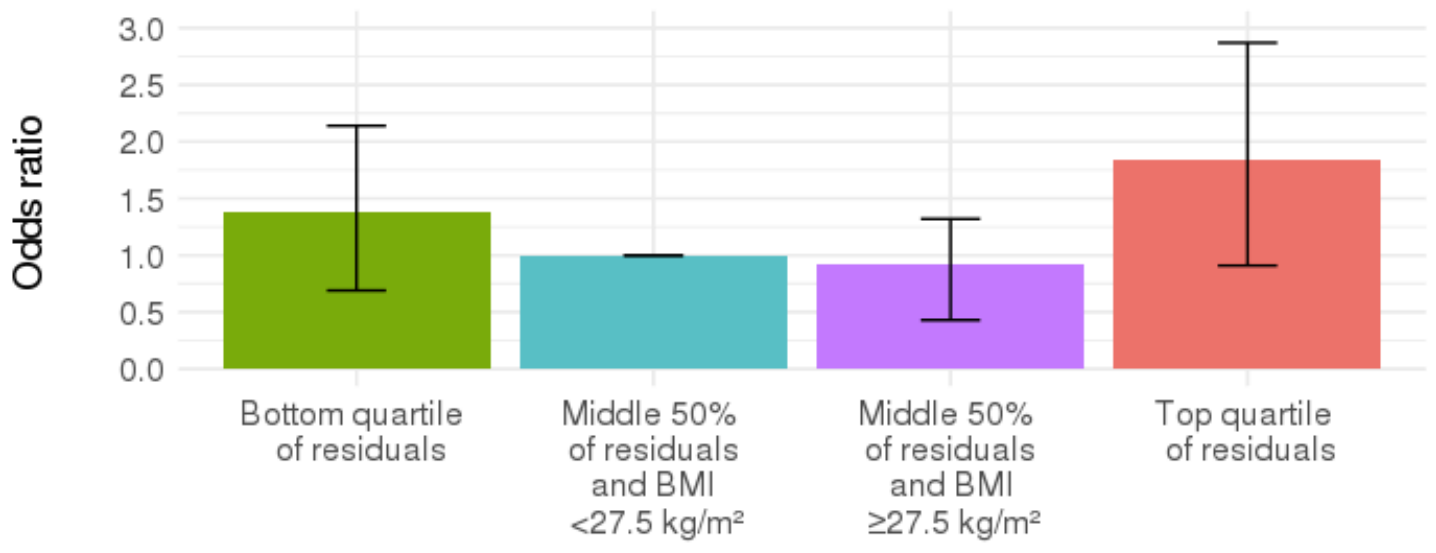

BMI-metabolic group

Appendix Figure $8 \mathrm{BMI}-$ metabolic groups and advanced prostate cancer. Panel A: Scatterplot of self-reported BMI vs. metabolic score (predicted BMI based on metabolites) with line of best fit drawn in black. Men were classified into BMI-metabolic groups based on being in the top quartile of residuals (red), bottom quartile of residuals (green), or in the middle $50 \%$ of residuals with a self-reported BMI $<25 \mathrm{~kg} / \mathrm{m}^{2}$ (blue) or $\geq 25 \mathrm{~kg} / \mathrm{m}^{2}$ (purple). Panel B: Odds ratios for advanced prostate cancer by BMI-metabolic group. Estimates adjusted for age, diabetes, physical activity, smoking status, family history of prostate cancer, and recent PSA screening. 


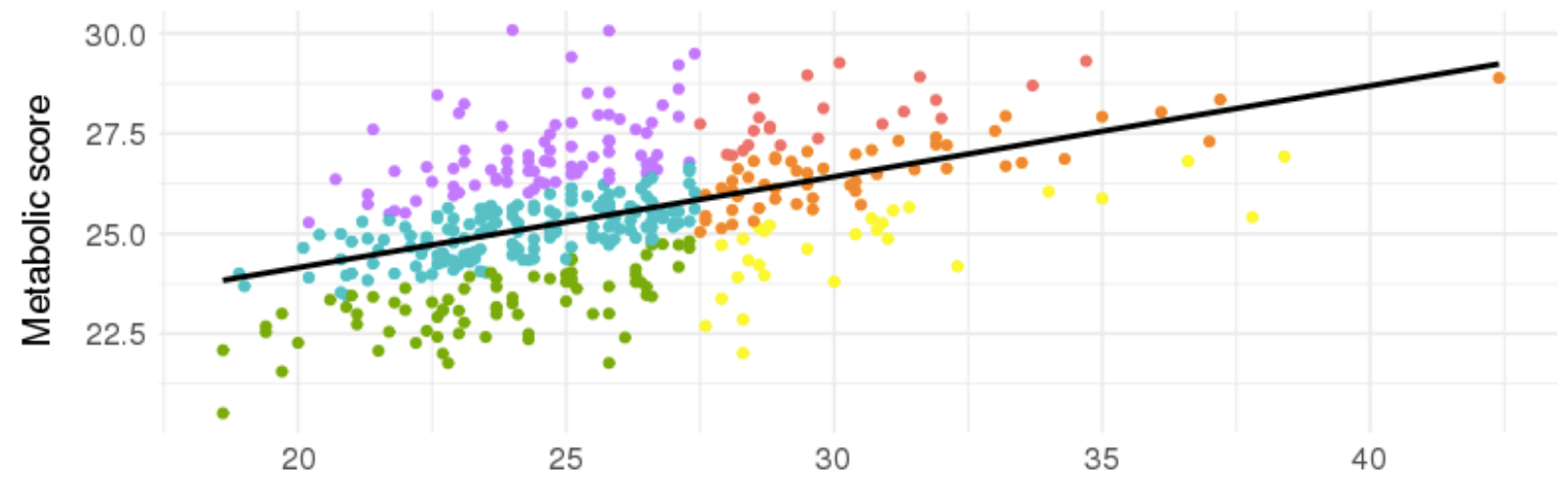

$\operatorname{BMI}\left(\mathrm{kg} / \mathrm{m}^{2}\right)$

B

Odds ratios for advanced prostate cancer by BMI-metabolic group

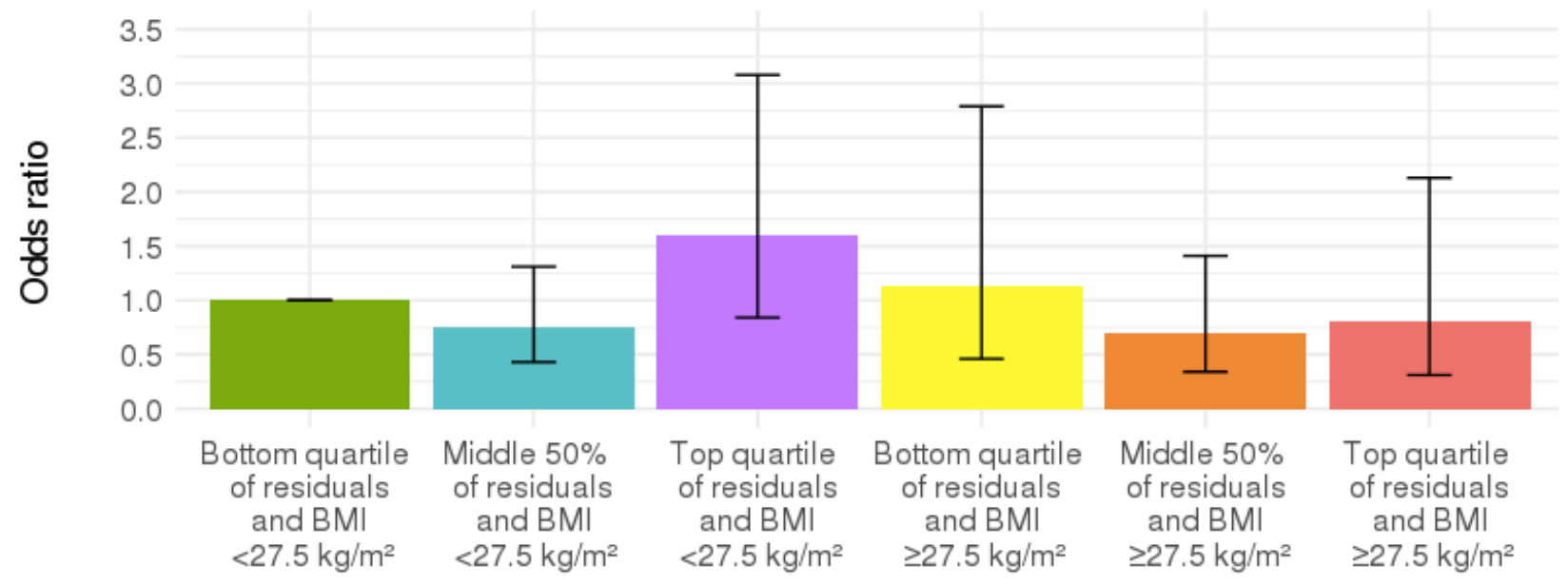

BMI-metabolic group

Appendix Figure 9 BMI-metabolic groups and advanced prostate cancer. Panel A: Scatterplot of self-reported BMI vs. metabolic score (predicted BMI based on metabolites) with line of best fit drawn in black. Men were classified into BMI-metabolic groups based on having a selfreported $\mathrm{BMl}<27.5 \mathrm{~kg} / \mathrm{m}^{2}$ and being in the bottom (green), middle $50 \%$ (blue), or top (purple) quartile of the residuals, or having a self-reported $\mathrm{BMI} \geq 27.5 \mathrm{~kg} / \mathrm{m}^{2}$ and being in the bottom (yellow), middle $50 \%$ (orange), or top (red) quartile of the residuals. Panel B: Odds ratios for advanced prostate cancer by BMI-metabolic group. Estimates adjusted for age, diabetes, physical activity, smoking status, family history of prostate cancer, and recent PSA screening. 
A

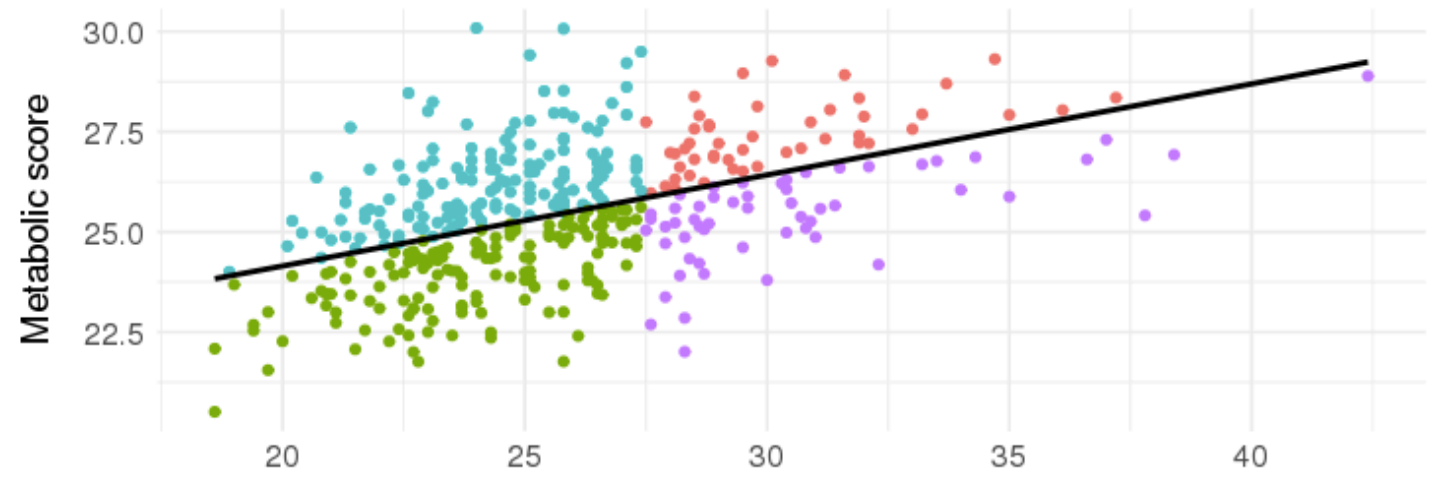

BMI $\left(\mathrm{kg} / \mathrm{m}^{2}\right)$

B Odds ratios for advanced prostate cancer by BMI-metabolic group

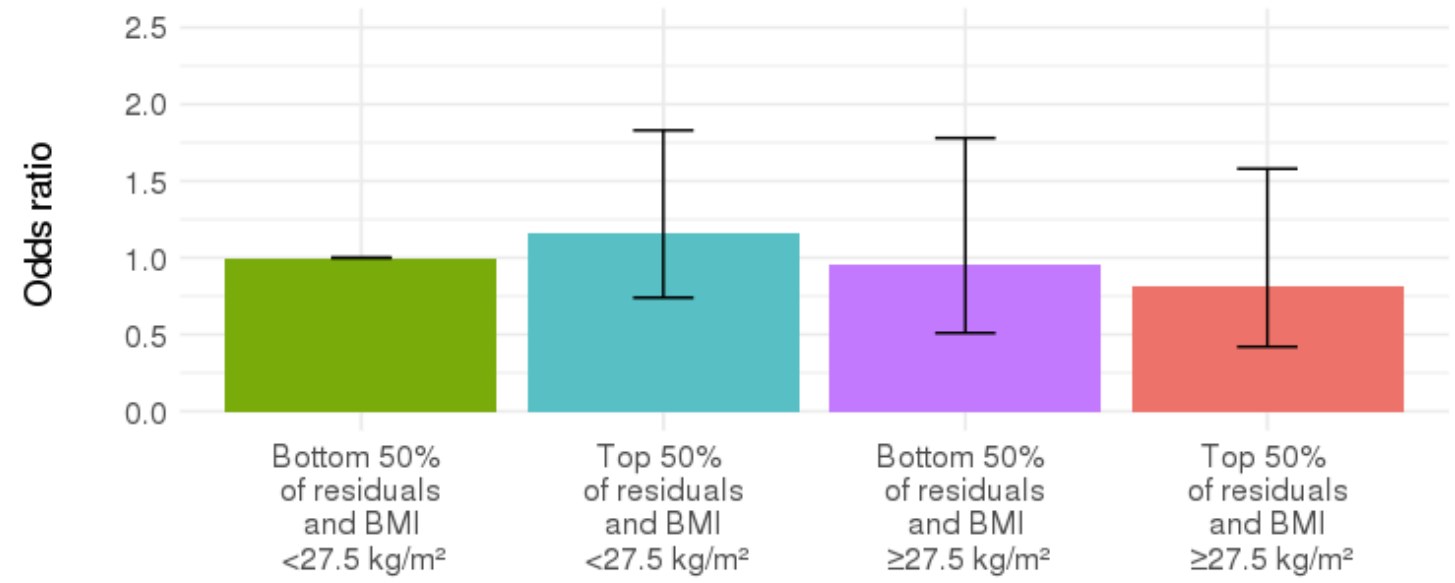

BMI-metabolic group

Appendix Figure 10 BMI-metabolic groups and advanced prostate cancer. Panel A:

Scatterplot of self-reported BMI vs. metabolic score (predicted BMI based on metabolites) with line of best fit drawn in black. Men were classified into BMl-metabolic groups based on having a self-reported BMI $<27.5 \mathrm{~kg} / \mathrm{m}^{2}$ and being in the bottom half (green) or top half (blue) of residuals, or having a self-reported $\mathrm{BMI} \geq 27.5 \mathrm{~kg} / \mathrm{m}^{2}$ and being in the bottom half (purple) or top half (red) of residuals. Panel B: Odds ratios for advanced prostate cancer by BMI-metabolic group. Estimates adjusted for age, diabetes, physical activity, smoking status, family history of prostate cancer, and recent PSA screening. 


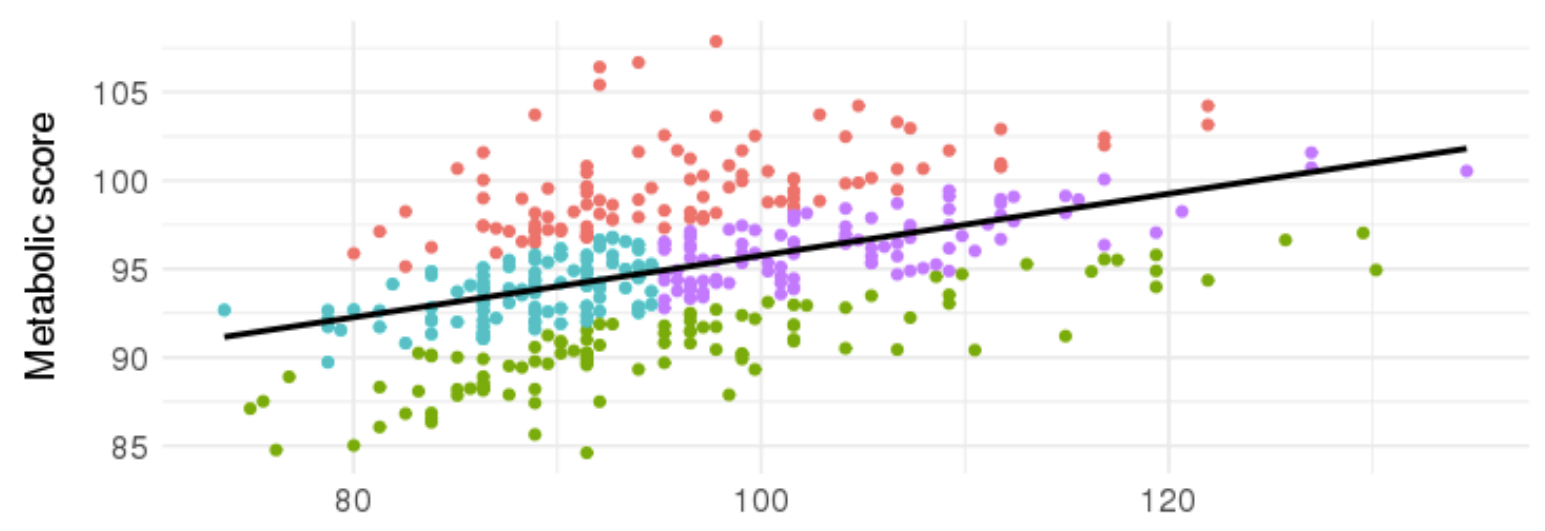

Waist circumference $(\mathrm{cm})$

B Odds ratios for advanced prostate cancer by waist circumference-metabolic group

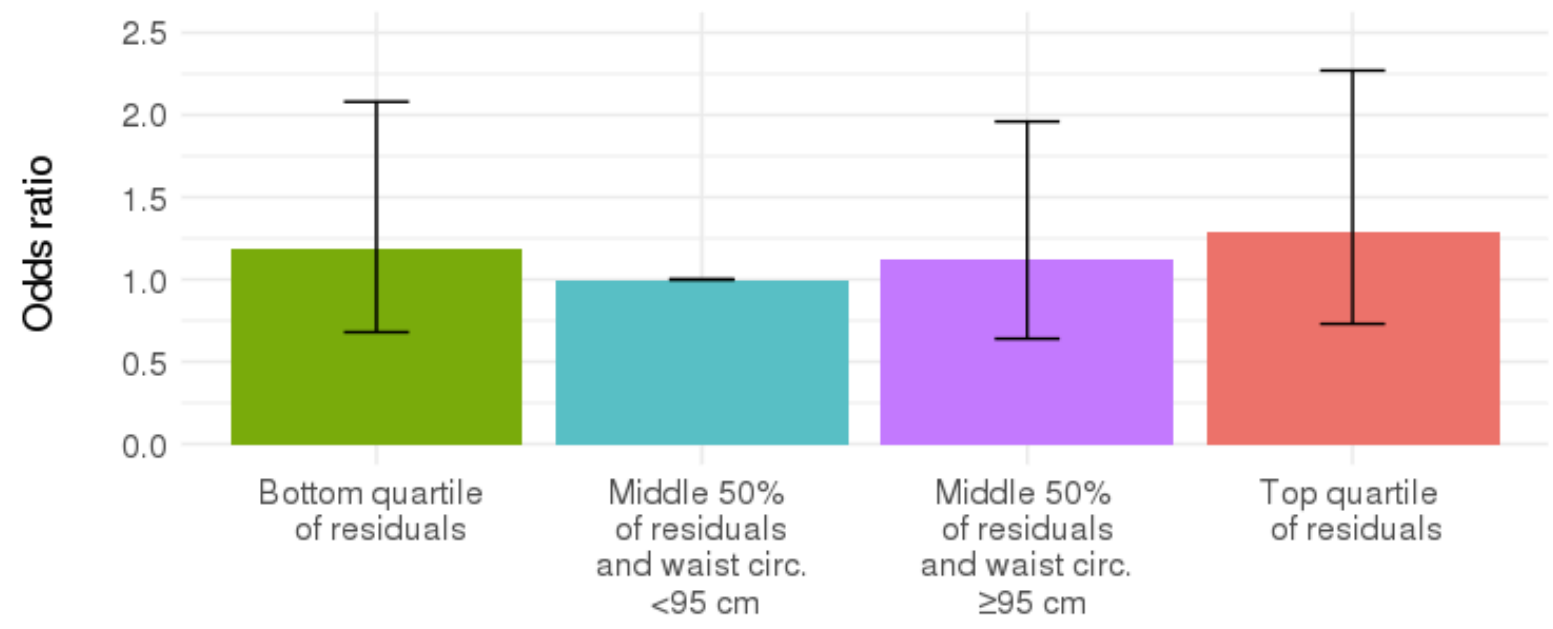

Waist circumference-metabolic group

Appendix Figure 11 Waist circumference-metabolic groups and advanced prostate cancer. Panel A: Scatterplot of self-reported waist circumference vs. metabolic score (predicted waist circumference based on metabolites) with line of best fit drawn in black. Men were classified into waist circumference-metabolic groups based on being in the top quartile of residuals (red), bottom quartile of residuals (green), or in the middle $50 \%$ of residuals with a self-reported waist circumference $<95 \mathrm{~cm}$ (blue) or $\geq 95 \mathrm{~cm}$ (purple). Panel B: Odds ratios for advanced prostate cancer by waist circumference-metabolic group. Estimates adjusted for age, diabetes, physical activity, smoking status, family history of prostate cancer, and recent PSA screening. 
A

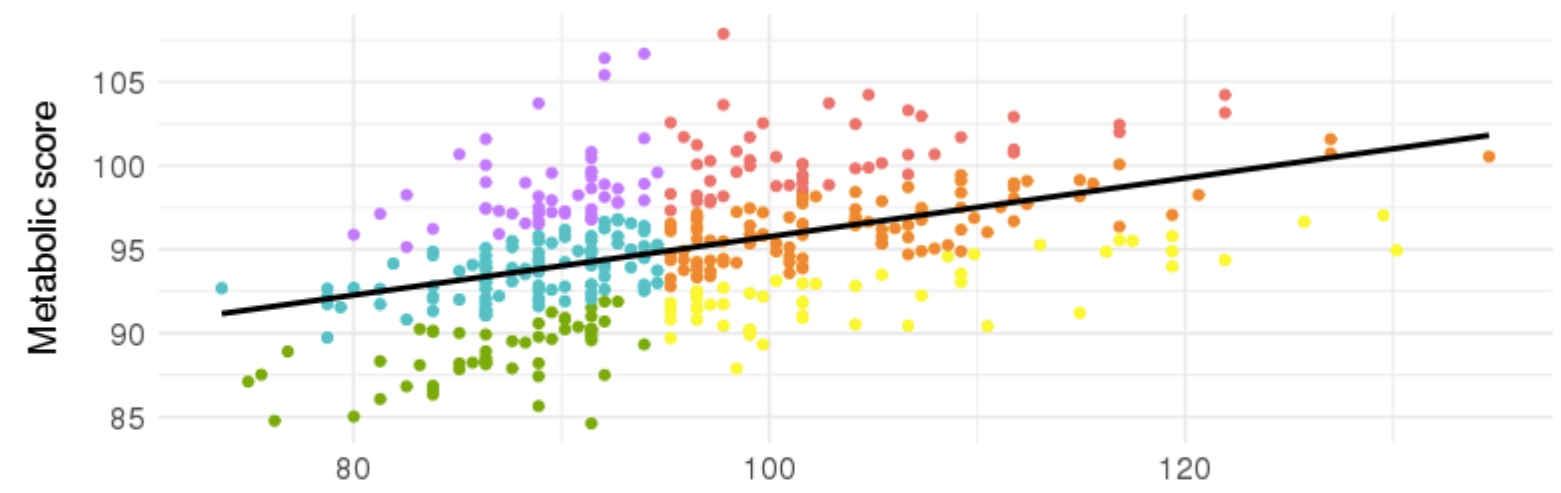

Waist circumference $(\mathrm{cm})$

B Odds ratios for advanced prostate cancer by waist circumference-metabolic group

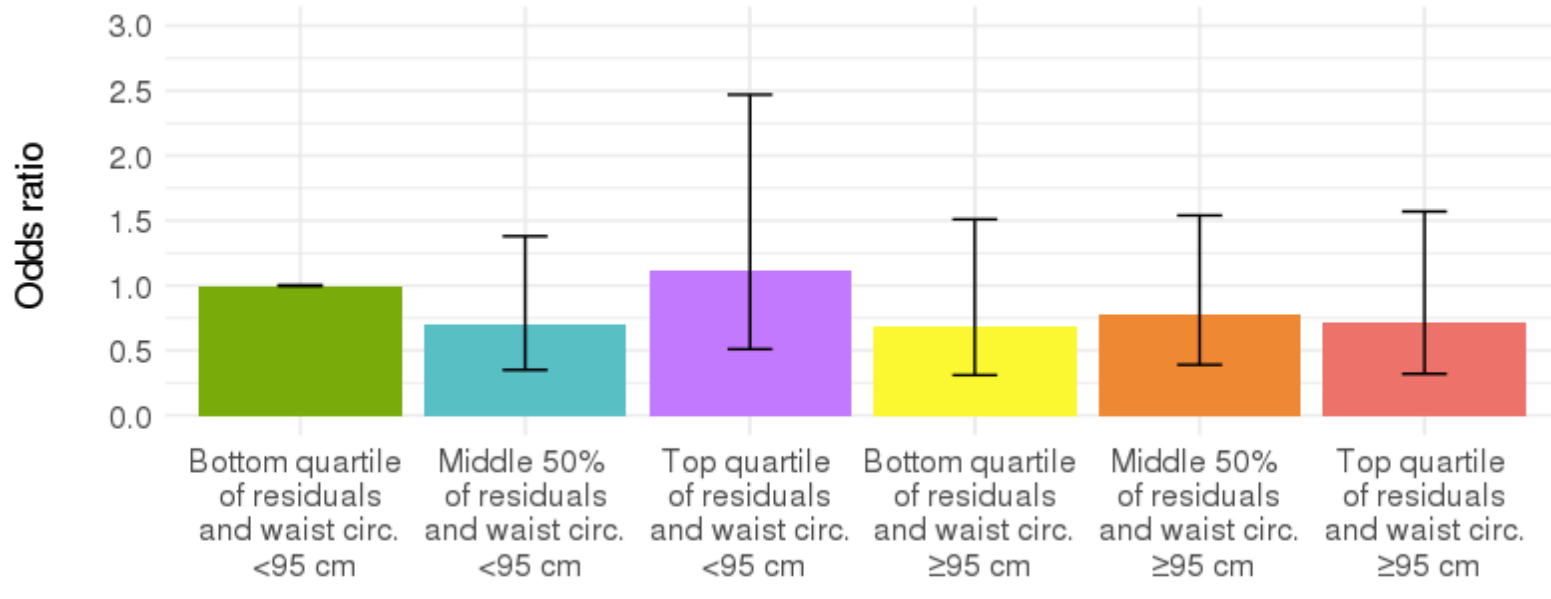

Waist circumference-metabolic group

Appendix Figure 12 Waist circumference-metabolic groups and advanced prostate cancer. Panel A: Scatterplot of self-reported waist circumference vs. metabolic score (predicted waist circumference based on metabolites) with line of best fit drawn in black. Men were classified into waist circumference-metabolic groups based on having a self-reported waist circumference $<95$ $\mathrm{cm}$ and being in the bottom (green), middle $50 \%$ (blue), or top (purple) quartile of the residuals, or having a self-reported waist circumference $\geq 95 \mathrm{~cm}$ and being in the bottom (yellow), middle $50 \%$ (orange), or top (red) quartile of the residuals. Panel B: Odds ratios for advanced prostate cancer by waist circumference-metabolic group. Estimates adjusted for age, diabetes, physical activity, smoking status, family history of prostate cancer, and recent PSA screening. 
A

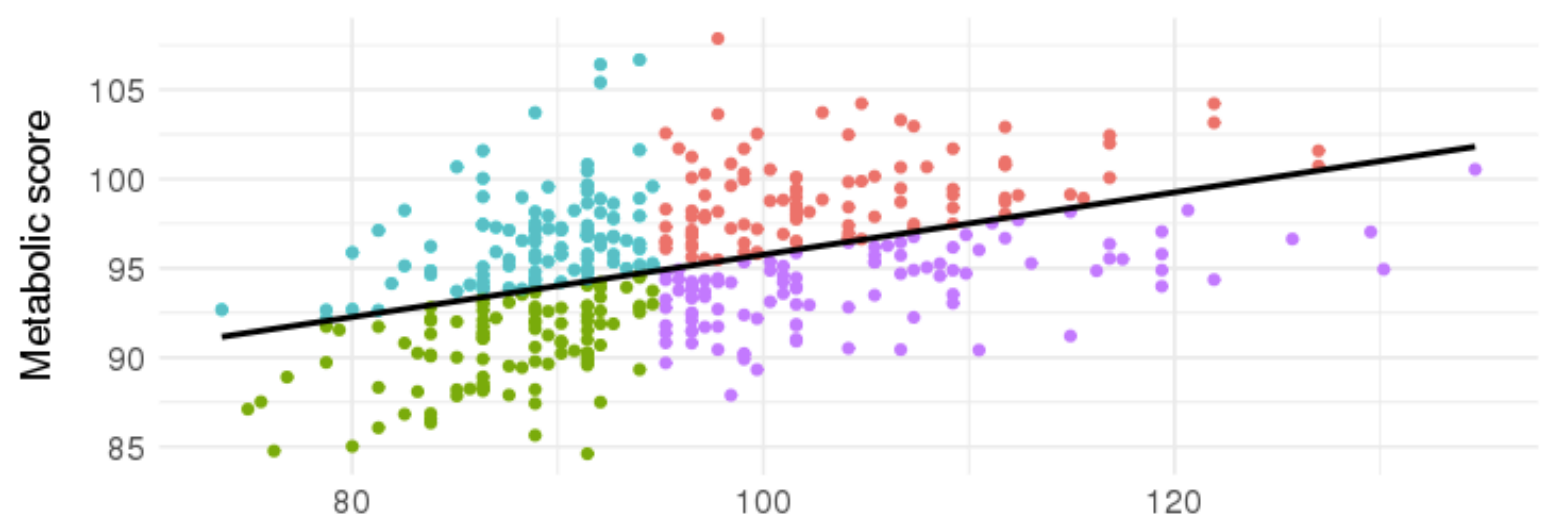

Waist circumference $(\mathrm{cm})$

B Odds ratios for advanced prostate cancer by waist circumference-metabolic group

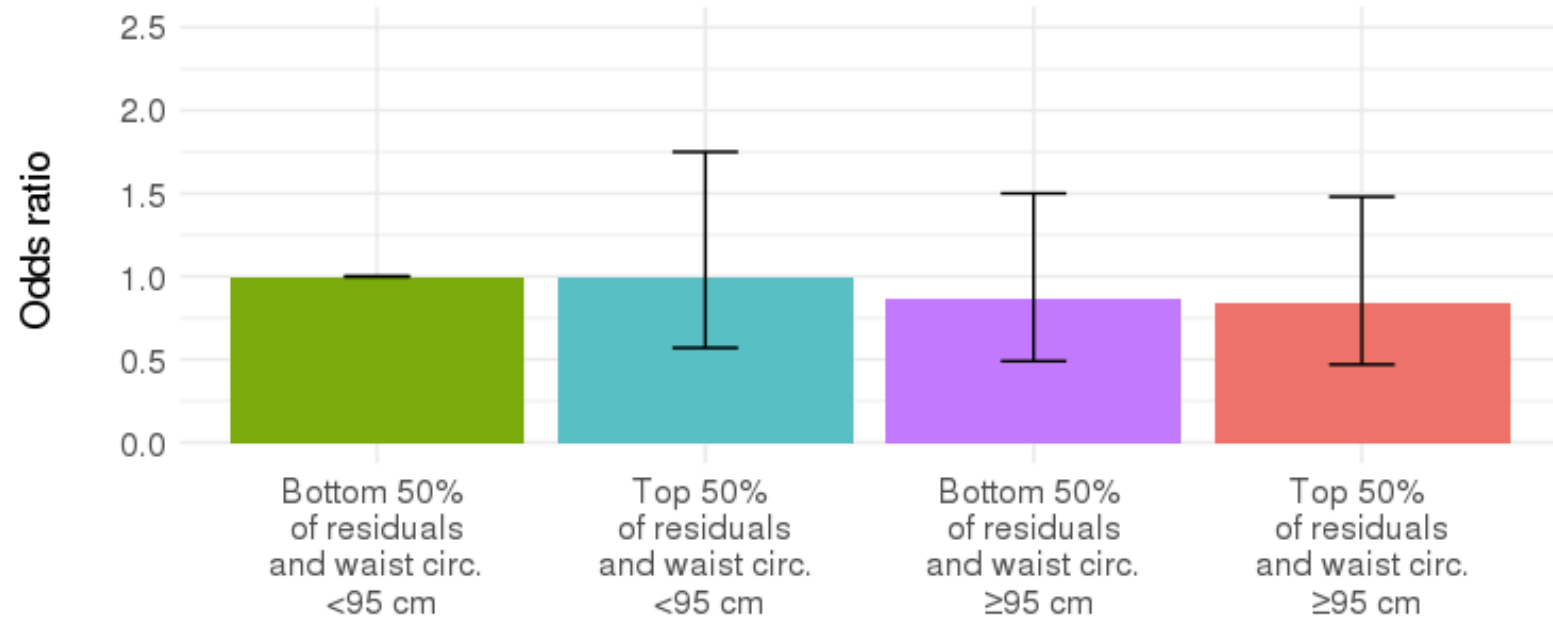

Waist circumference-metabolic group

Appendix Figure 13 Waist circumference-metabolic groups and advanced prostate cancer. Panel A: Scatterplot of self-reported waist circumference vs. metabolic score (predicted waist circumference based on metabolites) with line of best fit drawn in black. Men were classified into waist circumference-metabolic groups based on having a self-reported waist circumference $<95$ $\mathrm{cm}$ and being in the bottom half (green) or top half (blue) of residuals, or having a self-reported waist circumference $\geq 95 \mathrm{~cm}$ and being in the bottom half (purple) or top half (red) of residuals. Panel B: Odds ratios for advanced prostate cancer by waist circumference-metabolic group. Estimates adjusted for age, diabetes, physical activity, smoking status, family history of prostate cancer, and recent PSA screening. 
A

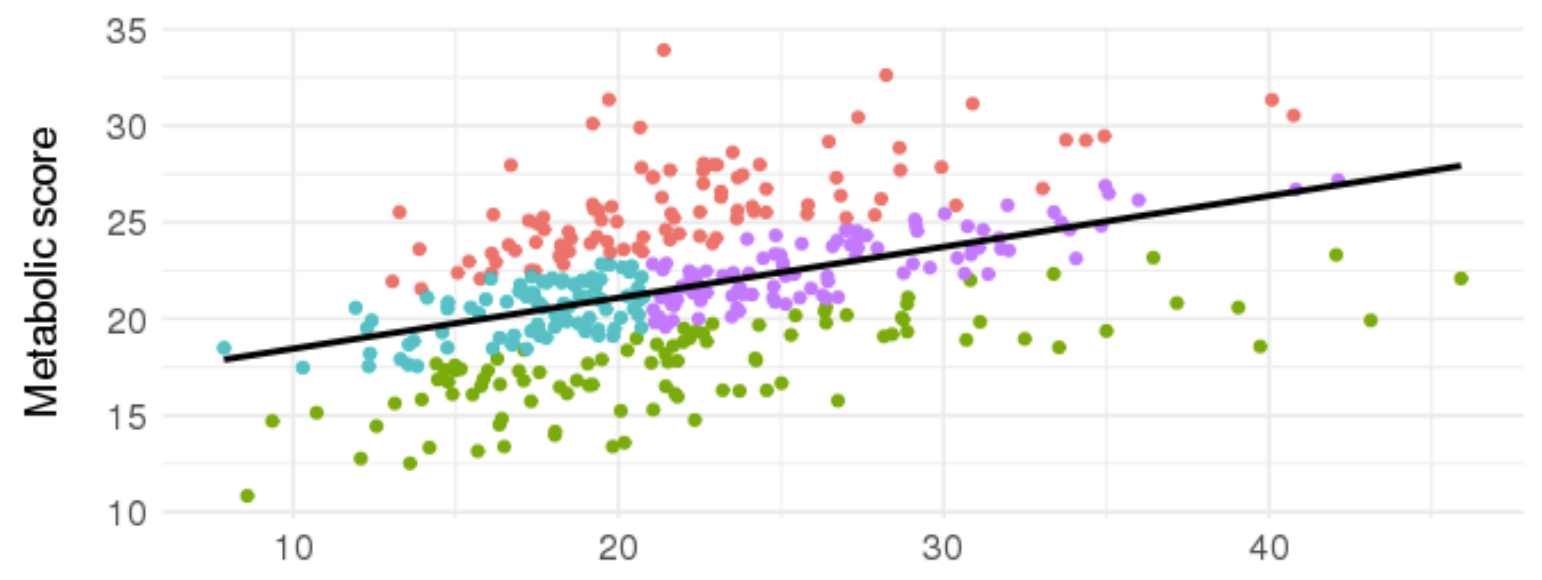

Fat mass $(\mathrm{kg})$

B Odds ratios for advanced prostate cancer by fat mass-metabolic group

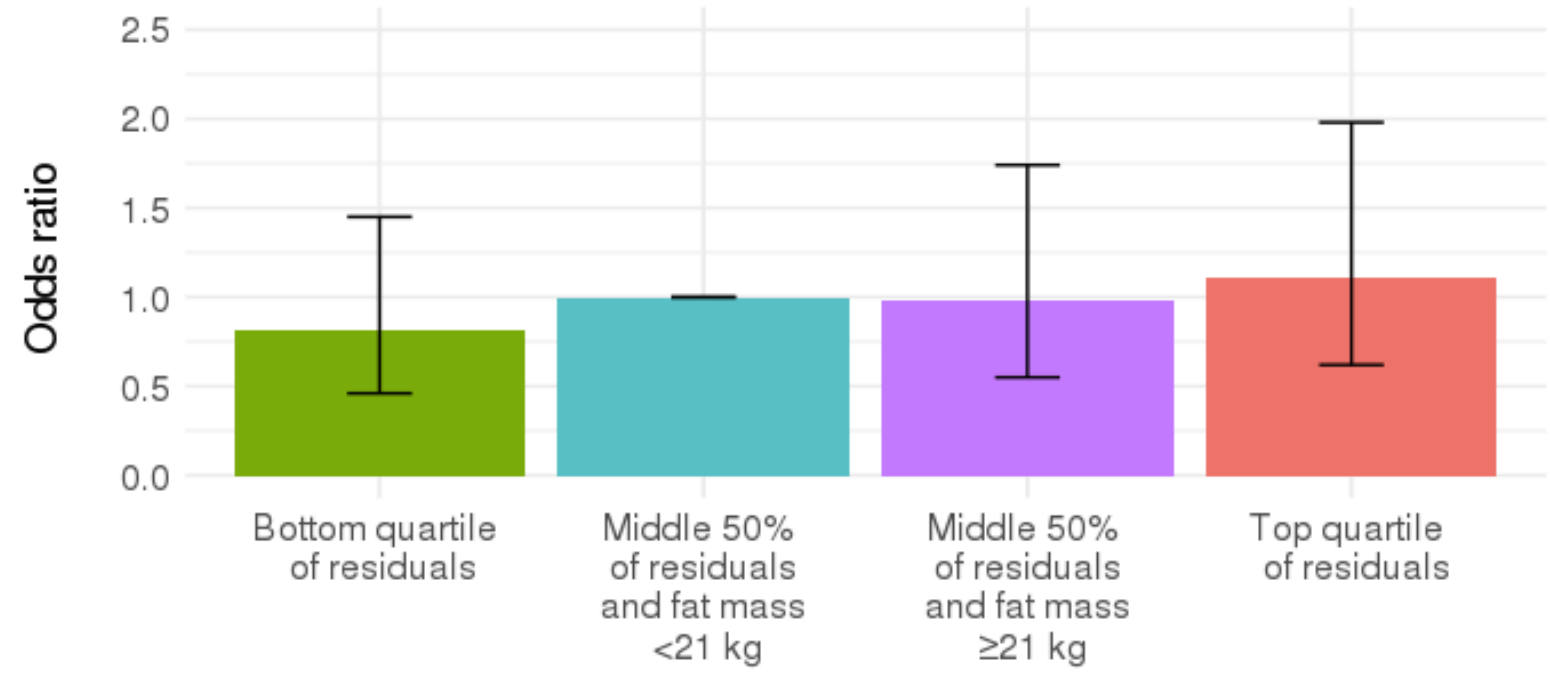

Fat mass-metabolic group

Appendix Figure 14 Fat mass-metabolic groups and advanced prostate cancer. Panel A: Scatterplot of derived fat mass based on a validated NHANES prediction model vs. metabolic score (predicted fat mass based on metabolites) with line of best fit drawn in black. Men were classified into fat mass-metabolic groups based on being in the top quartile of residuals (red), bottom quartile of residuals (green), or in the middle $50 \%$ of residuals with a derived fat mass $<21 \mathrm{~kg}$ (blue) or $\geq 21 \mathrm{~kg}$ (purple). Panel B: Odds ratios for advanced prostate cancer by fat mass-metabolic group. Estimates adjusted for age, diabetes, physical activity, smoking status, family history of prostate cancer, and recent PSA screening. 


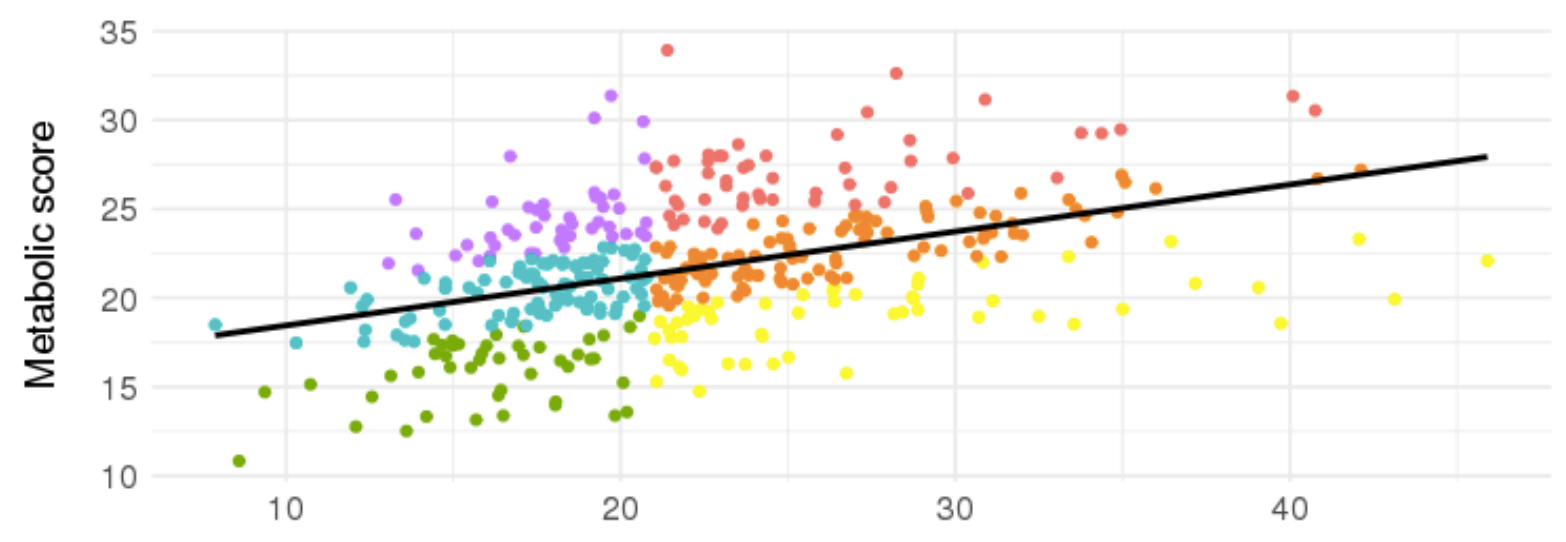

Fat mass $(\mathrm{kg})$

B Odds ratios for advanced prostate cancer by fat mass-metabolic group

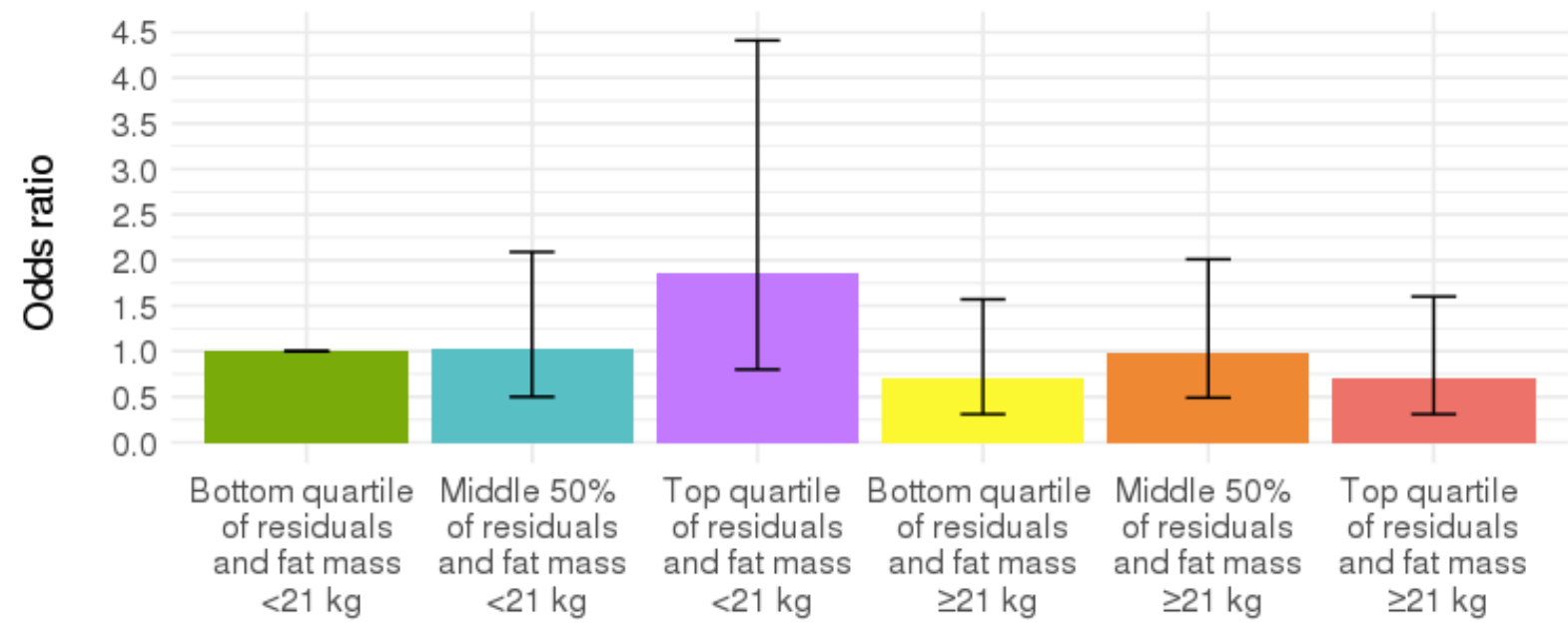

Fat mass-metabolic group

Appendix Figure 15 Fat mass-metabolic groups and advanced prostate cancer. Panel A: Scatterplot of fat mass based on a validated NHANES prediction model vs. metabolic score (predicted fat mass based on metabolites) with line of best fit drawn in black. Men were classified into fat mass-metabolic groups based on having a derived fat mass $<21 \mathrm{~kg}$ and being in the bottom (green), middle 50\% (blue), or top (purple) quartile of the residuals, or having a derived fat mass $\geq 21 \mathrm{~kg}$ and being in the bottom (yellow), middle $50 \%$ (orange), or top (red) quartile of the residuals. Panel B: Odds ratios for advanced prostate cancer by fat massmetabolic group. Estimates adjusted for age, diabetes, physical activity, smoking status, family history of prostate cancer, and recent PSA screening. 
A

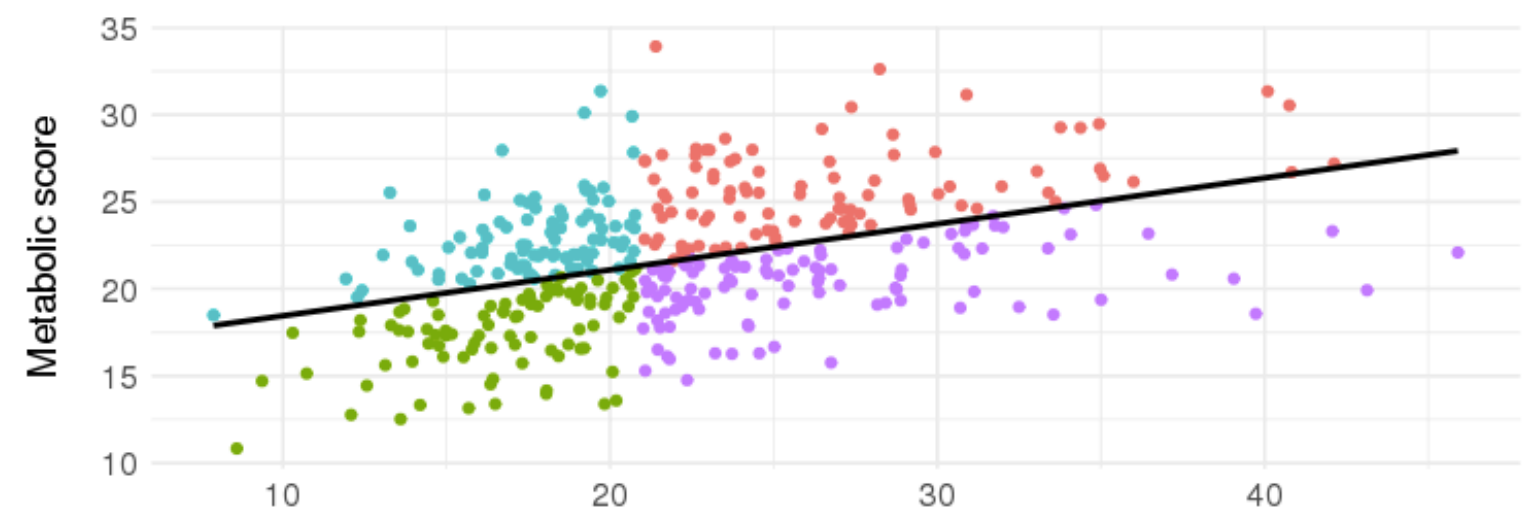

Fat mass $(\mathrm{kg})$

B Odds ratios for advanced prostate cancer by fat mass-metabolic group

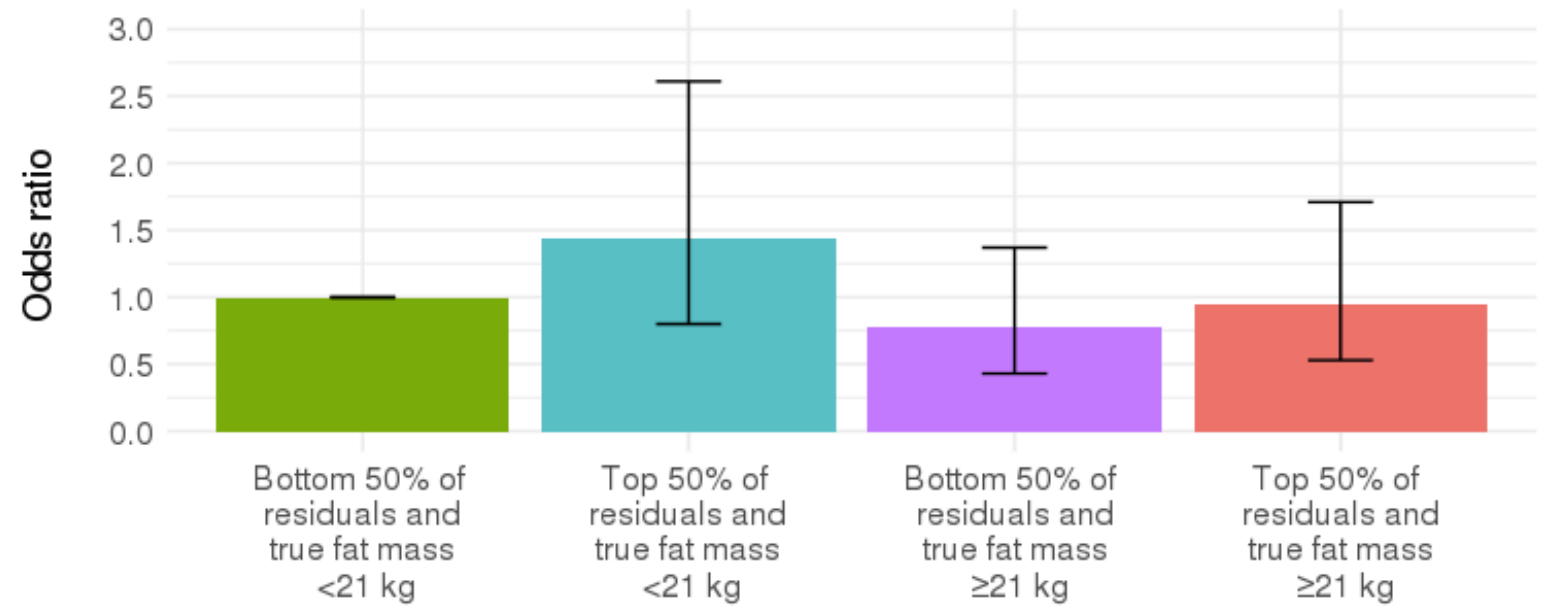

Fat mass-metabolic group

Appendix Figure 16 Fat mass-metabolic groups and advanced prostate cancer. Panel A: Scatterplot of fat mass based on a validated NHANES prediction model vs. metabolic score (predicted fat mass based on metabolites) with line of best fit drawn in black. Men were classified into fat mass-metabolic groups based on having a derived fat mass $<21 \mathrm{~kg}$ and being in the bottom half (green) or top half (blue) of residuals, or having a derived fat mass $\geq 21 \mathrm{~kg}$ and being in the bottom half (purple) or top half (red) of residuals. Panel B: Odds ratios for advanced prostate cancer by fat mass-metabolic group. Estimates adjusted for age, diabetes, physical activity, smoking status, family history of prostate cancer, and recent PSA screening. 
Page intentionally left blank 